\title{
HIGH-RESOLUTION SPECTROSCOPY OF EXTREMELY METAL-POOR STARS IN THE LEAST EVOLVED GALAXIES: URSA MAJOR II and COMA BERENICES*
}

\author{
Anna Frebel ${ }^{1,2}$, Joshua D. Simon ${ }^{3}$, Marla Geha $^{4}$, and Beth Willman ${ }^{5}$ \\ ${ }^{1}$ Harvard-Smithsonian Center for Astrophysics, Cambridge, MA 02138, USA; afrebel@cfa.harvard.edu \\ ${ }^{2}$ McDonald Observatory, University of Texas at Austin, Austin, TX 78712, USA \\ ${ }^{3}$ Observatories of the Carnegie Institution of Washington, Pasadena, CA 91101, USA; jsimon@ociw.edu \\ ${ }^{4}$ Astronomy Department, Yale University, New Haven, CT 06520 USA; marla.geha@yale.edu \\ ${ }^{5}$ Haverford College, Haverford, PA 19041, USA; bwillman@ haverford.edu \\ Received 2009 February 13; accepted 2009 November 11; published 2009 December 14
}

\begin{abstract}
We present spectra of six metal-poor stars in two of the ultra-faint dwarf galaxies orbiting the Milky Way (MW), Ursa Major II, and Coma Berenices obtained with the Keck/High Resolution Echelle Spectrometer (HIRES). These observations include the first high-resolution spectroscopic observations of extremely metal-poor $([\mathrm{Fe} / \mathrm{H}]<-3.0)$ stars not belonging to the MW halo field star population. We obtain abundance measurements and upper limits for 26 elements between carbon and europium. The entire sample of stars spans a range of $-3.2<[\mathrm{Fe} / \mathrm{H}]<-2.3$, and we confirm that each galaxy contains a large intrinsic spread of Fe abundances. A comparison with MW halo stars of similar metallicities reveals substantial agreement between the abundance patterns of the ultra-faint dwarf galaxies and the MW halo for the light, $\alpha$, and iron-peak elements ( $\mathrm{C}$ to $\mathrm{Zn}$ ). This agreement contrasts with the results of earlier studies of more metal-rich stars $(-2.5 \lesssim[\mathrm{Fe} / \mathrm{H}] \lesssim-1.0)$ in more luminous dwarf spheroidal galaxies, which found significant abundance discrepancies with respect to the MW halo data. The abundances of neutron-capture elements ( $\mathrm{Sr}$ to $\mathrm{Eu}$ ) in the ultra-faint dwarf galaxies are extremely low, consistent with the most metal-poor halo stars, but not with the typical halo abundance pattern at $[\mathrm{Fe} / \mathrm{H}] \gtrsim-3.0$. Not only are our results broadly consistent with a galaxy formation model that predicts that massive dwarf galaxies are the source of the metal-rich component $([\mathrm{Fe} / \mathrm{H}]>-2.5)$ of the MW halo, but they also suggest that the faintest known dwarfs may be the primary contributors to the metal-poor end of the MW halo metallicity distribution.
\end{abstract}

Key words: early universe - galaxies: dwarf - Galaxy: halo - Local Group - stars: abundances stars: Population II

Online-only material: color figures and machine-readable table

\section{INTRODUCTION}

Dwarf spheroidal (dSph) galaxies are among the most metalpoor stellar systems in the local universe (Mateo 1998), and the recently discovered "ultra-faint" $\left(M_{V}>\sim 8\right)$ dwarf galaxies (Willman et al. 2005a, 2005b; Zucker et al. 2006a, 2006b; Belokurov et al. 2006, 2007; Sakamoto \& Hasegawa 2006; Irwin et al. 2007; Walsh et al. 2007; Belokurov et al. 2008) are the least chemically enriched systems yet found (Muñoz et al. 2006; Simon \& Geha 2007; Kirby et al. 2008; Geha et al. 2009). The mean metallicity $[\mathrm{Fe} / \mathrm{H}]^{6}$ of the 12 Milky Way (MW) ultra-faint dwarf galaxies observed so far is $[\mathrm{Fe} / \mathrm{H}]=-2.3$ (Kirby et al. 2008), and the most metal-poor of these have lower metallicities than any known globular cluster (Harris 1997). The ultra-faint dwarf galaxies are highly dark matter-dominated (Martin et al. 2007; Simon \& Geha 2007; Strigari et al. 2008; Geha et al. 2009) and lie on the extension of the metallicity-luminosity relationship and other scaling relations established by brighter dSph galaxies (Kirby et al. 2008; Peñarrubia et al. 2008). Thus, these objects appear to represent the extreme limit of the galaxy formation process.

\footnotetext{
* Based on observations obtained at the W. M. Keck Observatory, which is operated jointly by the California Institute of Technology and the University of California, and the National Aeronautics and Space Administration.

6 Throughout this paper, we assume that the Fe abundance traces the overall metallicity $Z$ of a star. We thus use the terms metallicity, Fe abundance, and $[\mathrm{Fe} / \mathrm{H}]$ interchangeably, where $[\mathrm{A} / \mathrm{B}]=\log \left(N_{\mathrm{A}} / N_{\mathrm{B}}\right)-\log \left(N_{\mathrm{A}} / N_{\mathrm{B}}\right)_{\odot}$ for the number $N$ of atoms of elements $\mathrm{A}$ and $\mathrm{B}$
}

Detailed chemical abundance measurements of individual stars in dwarf galaxies can provide a unique window into how star formation and chemical enrichment proceeded in the early universe. Such "stellar archaeology" is a powerful tool for recovering the chemical composition of the stellar birth cloud and revealing how the star-forming gas was enriched by previous generation(s) of stars. The very low Fe abundances seen in the ultra-faint dwarf galaxies suggest that perhaps only one or a few generations of star formation occurred before the birth of the stars observed today. These galaxies therefore afford us an unusually clear glimpse of the nucleosynthetic products of some of the first stars.

The past decade has provided a wealth of new information about chemical abundances in dSphs in general (e.g., Shetrone et al. 1998, 2001, 2003; Tolstoy et al. 2003; Venn et al. 2004; Geisler et al. 2005), but the ultra-faint dwarf galaxies were discovered too recently to have been included in these studies. Two of the major results from work on the brighter dSphs were that the abundance patterns of dSph stars (most notably the $[\alpha / \mathrm{Fe}]$ ratios) differ significantly from those seen in the stellar halo of the MW (Venn et al. 2004, and references therein) and that the $\mathrm{dSphs}$ seemed to lack the extremely metal-poor $([\mathrm{Fe} / \mathrm{H}]<-3)$ stars (Helmi et al. 2006) that are known to be present in the MW halo (e.g., Beers \& Christlieb 2005). Although comparisons to the most recent unbiased determination of the halo metallicity distribution function (MDF) now indicate that the $\mathrm{dSph}$ metallicity distributions may be reasonably consistent with the halo (Schöerck et al. 2009), it is still the case 
Table 1

Photometry

\begin{tabular}{llccccr}
\hline \hline \multicolumn{1}{c}{ Galaxy } & SDSS Designation & Star & $V$ & $r$ & $g-r$ & $M_{r}$ \\
\hline Ursa Major II & SDSS J084954+630822 & UMa II-S1 & 18.13 & 17.85 & 0.68 & 0.08 \\
Ursa Major II & SDSS J085002+631333 & UMa II-NM & 16.83 & 16.54 & 0.70 & -1.15 \\
Ursa Major II & SDSS J085234+630501 & UMa II-S2 & 17.66 & 17.37 & 0.72 & -0.38 \\
Ursa Major II & SDSS J085259+630555 & UMa II-S3 & 16.79 & 16.43 & 0.86 & -1.33 \\
Coma Berenices & SDSS J122643+235702 & ComBer-S1 & 18.12 & 17.89 & 0.58 & -0.29 \\
Coma Berenices & SDSS J122655+235610 & ComBer-S2 & 17.50 & 17.24 & 0.65 & -0.94 \\
Coma Berenices & SDSS J122657+235611 & ComBer-S3 & 18.02 & 17.77 & 0.62 & -0.41 \\
\hline
\end{tabular}

Note. UMa II-NM was observed but was later determined not to be a member of UMa II.

that no extremely metal-poor stars have been identified in the brightest dSphs.

These findings were initially interpreted as a challenge to hierarchical formation scenarios for the MW; if the stellar halo is built up by the destruction of dwarf galaxies (e.g., Searle \& Zinn 1978), then one might naively expect stars in dwarf galaxies to have similar properties to halo stars. Subsequently, more sophisticated analyses combining $N$-body simulations and semi-analytic chemical evolution models demonstrated that the $[\alpha / \mathrm{Fe}]$ discrepancy is in fact a natural by-product of stellar halo formation in a hierarchical universe, because the bulk of the MW halo had its origin in satellites much more massive than the presently observed dSphs (Robertson et al. 2005; Bullock \& Johnston 2005; Font et al. 2006; Johnston et al. 2008). However, this does not solve the mystery of the missing extremely metalpoor stars in dSphs; such stars are observed in the halo, so they must have come from somewhere. This remaining problem, plus the recent discovery of extremely metal-poor stars in the ultrafaint dwarf galaxies (Kirby et al. 2008), strongly motivates more detailed abundance studies of these galaxies.

Furthermore, the large majority of stars with published highresolution abundance measurements are relatively metal-richonly 12 out of 49 have metallicities below $[\mathrm{Fe} / \mathrm{H}]=-2.0$ (Shetrone et al. 1998, 2001, 2003; Sadakane et al. 2004; Geisler et al. 2005; Koch et al. 2008a, 2008b). ${ }^{7}$ These stars are therefore unlikely to be representative of the earliest generations of star formation in dSphs. The most metal-poor dSph star observed at high resolution so far, Dra 119 in the Draco dSph (Shetrone et al. 1998; Fulbright et al. 2004), has a metallicity of $[\mathrm{Fe} / \mathrm{H}]=-2.95$. This star shows an enhancement of $\alpha$ elements and a lack of neutron-capture elements, hinting that perhaps the most metal-poor components of the MW halo, and the dwarf galaxies are actually similar, and the disagreements only set in at higher metallicities.

The first high-resolution spectroscopy of stars in the ultrafaint dwarf galaxies was presented by Koch et al. (2008b), who observed two stars in the Hercules (Her) dwarf. These stars have moderately low metallicities $([\mathrm{Fe} / \mathrm{H}] \sim-2)$, strong enhancements of the explosive $\alpha$-elements magnesium and oxygen, and no detected heavy elements. With the exception of unusual $[\mathrm{Mg} / \mathrm{Ca}]$ ratios, Koch et al. conclude that the abundance pattern in Her is comparable to that of extremely low-metallicity MW halo stars-but quite different from the typical abundances of halo stars at $[\mathrm{Fe} / \mathrm{H}]=-2$.

Here we present the first detailed chemical abundance measurements for two more ultra-faint dwarf galaxies, Ursa Major II

\footnotetext{
7 This compilation excludes 51 stars that have been observed in Sagittarius (Sgr), all of which have $[\mathrm{Fe} / \mathrm{H}]>-1.6$ (Bonifacio et al. 2000, 2004;

McWilliam et al. 2003; Monaco et al. 2005; Chou 2007), as well as studies of stars in the Sgr stream and Sgr and Fornax globular clusters.
}

(UMa II) and Coma Berenices (ComBer). UMa II $\left(M_{V}=-4.2\right)$ and $\operatorname{ComBer}\left(M_{V}=-4.1\right)$ are an order of magnitude less luminous than Her, and medium-resolution spectra indicate that their brightest stars have significantly lower Fe abundances than the Koch et al. (2008b) targets in Hercules. Using the High Resolution Echelle Spectrometer (HIRES) of the Keck telescope, we have obtained high-resolution spectra for the six brightest known member stars in these two galaxies (three stars in each galaxy). From these data, we are able to measure carbon, iron-peak and $\alpha$-element abundances, as well as neutron-capture species such as $\mathrm{Ba}$ and Sr. Two of the three stars observed in UMa II have $[\mathrm{Fe} / \mathrm{H}]<-3.0$, making them the most metal-poor stars studied with high-resolution spectroscopy that do not belong to the MW.

This paper is organized as follows. In Section 2, we describe the observations and our analysis techniques. In Section 3, the details of the elemental abundance determinations are presented as well as a comparison of our metal-poor stars with MW halo stars in a similar metallicity range. We interpret our results within the context of previous observations of dSphs and the hierarchical buildup of the MW halo in Section 4 and summarize our main findings in Section 5.

\section{OBSERVATIONS AND DATA ANALYSIS}

\subsection{Target Selection and Observations}

Obtaining detailed chemical abundances for stars in the ultrafaint dwarf galaxies is challenging due to their distances (the closest is located at $23 \mathrm{kpc}$ ) and their poorly populated red giant branches (RGBs). The Simon \& Geha (2007) spectroscopic data set contains a total of nine stars brighter than $r=18$ that are classified as ultra-faint dwarf galaxy members. These stars are just bright enough for reasonable observations at high spectral resolution with the largest available telescopes. The photometry of the observed stars is listed in Table 1. The $r$ magnitudes and $g-r$ colors were obtained from the updated SDSS DR7 (Abazajian et al. 2009), while the $V$ magnitudes were determined from $g$ and $r$ using the conversions given by Smith et al. (2002). Four of the target stars are located in UMa II and three are in ComBer; our two additional targets in Ursa Major I were abandoned because of worse than average observing conditions. Calcium triplet metallicity estimates for these stars indicate metallicities between $[\mathrm{Fe} / \mathrm{H}]=-2.6$ and -1.5 . The Kirby et al. (2008) spectral synthesis method yields even lower metallicities of $-3.0 \leqslant[\mathrm{Fe} / \mathrm{H}] \leqslant-2.3$, suggesting that our targets are some of the most metal-poor stars yet observed in dwarf galaxies.

We observed the target stars with the HIRES spectrograph (Vogt et al. 1994) on the Keck I telescope on 2008 February 22-24. Observing conditions during the run were generally clear, with an average seeing of $1^{\prime \prime} .0$. We used a $7{ }^{\prime \prime} .0 \times$ 
Table 2

Observing Details

\begin{tabular}{|c|c|c|c|c|c|c|c|c|c|c|}
\hline Star & $\begin{array}{c}\alpha \\
(\mathrm{J} 2000)\end{array}$ & $\begin{array}{c}\delta \\
(\mathrm{J} 2000) \\
\end{array}$ & JD & $\begin{array}{c}t_{\exp } \\
\mathrm{hr}\end{array}$ & $\begin{array}{c}\mathrm{S} / \mathrm{N} \\
5000 \AA \\
\end{array}$ & $\begin{array}{c}\mathrm{S} / \mathrm{N} \\
6000 \AA \\
\end{array}$ & $\begin{array}{c}\mathrm{S} / \mathrm{N} \\
6500 \AA \\
\end{array}$ & $\begin{array}{r}v_{\text {rad,HIRES }} \\
\left(\mathrm{km} \mathrm{s}^{-1}\right)\end{array}$ & $\begin{array}{c}v_{\text {rad,DEIMOS }} \\
\left(\mathrm{km} \mathrm{s}^{-1}\right) \\
\end{array}$ & Comment \\
\hline UMa II-S1 & 084953.46 & 630821.94 & 2454520.8 & 5.00 & 20 & 30 & 38 & $-124.5 \pm 0.3$ & $-121.5 \pm 2.2$ & target \\
\hline UMa II-NM & 085001.84 & 631333.05 & 2454521.0 & 1.50 & 33 & 33 & 42 & $-112.0 \pm 0.2$ & $-111.0 \pm 2.2$ & target \\
\hline UMa II-S2 & 085233.50 & 630501.33 & 2454519.8 & 3.00 & 24 & 37 & 42 & $-110.6 \pm 0.3$ & $-107.5 \pm 2.2$ & target \\
\hline UMa II-S3 & 085259.07 & 630554.81 & 2454519.8 & 1.00 & 20 & 27 & 30 & $-119.8 \pm 0.2$ & $-102.6 \pm 2.2$ & target \\
\hline ComBer-S1 & 122643.47 & 235702.47 & 2454522.0 & 5.29 & 22 & 27 & 29 & $93.8 \pm 0.3$ & $97.3 \pm 2.2$ & target \\
\hline ComBer-S2 & 122655.46 & 235609.83 & 2454520.0 & 5.25 & 23 & 28 & 30 & $96.4 \pm 0.2$ & $97.5 \pm 2.2$ & target \\
\hline ComBer-S3 & 122656.67 & 235611.84 & 2454521.1 & 2.83 & 33 & 47 & 51 & $99.0 \pm 0.3$ & $102.6 \pm 2.2$ & target \\
\hline HD 122563 & 140231.85 & 094109.94 & 2454521.9 & 0.01 & 530 & 650 & 750 & $-25.1 \pm 0.2$ & $\ldots$ & standard \\
\hline
\end{tabular}

Note. The S/N measurements are for $\sim 22 \mathrm{~m} \AA$ ("blue" CCD), $\sim 26 \mathrm{~m} \AA$ ("green" CCD), and $\sim 28 \mathrm{~m} \AA$ ("green" CCD) pixel sizes, respectively.

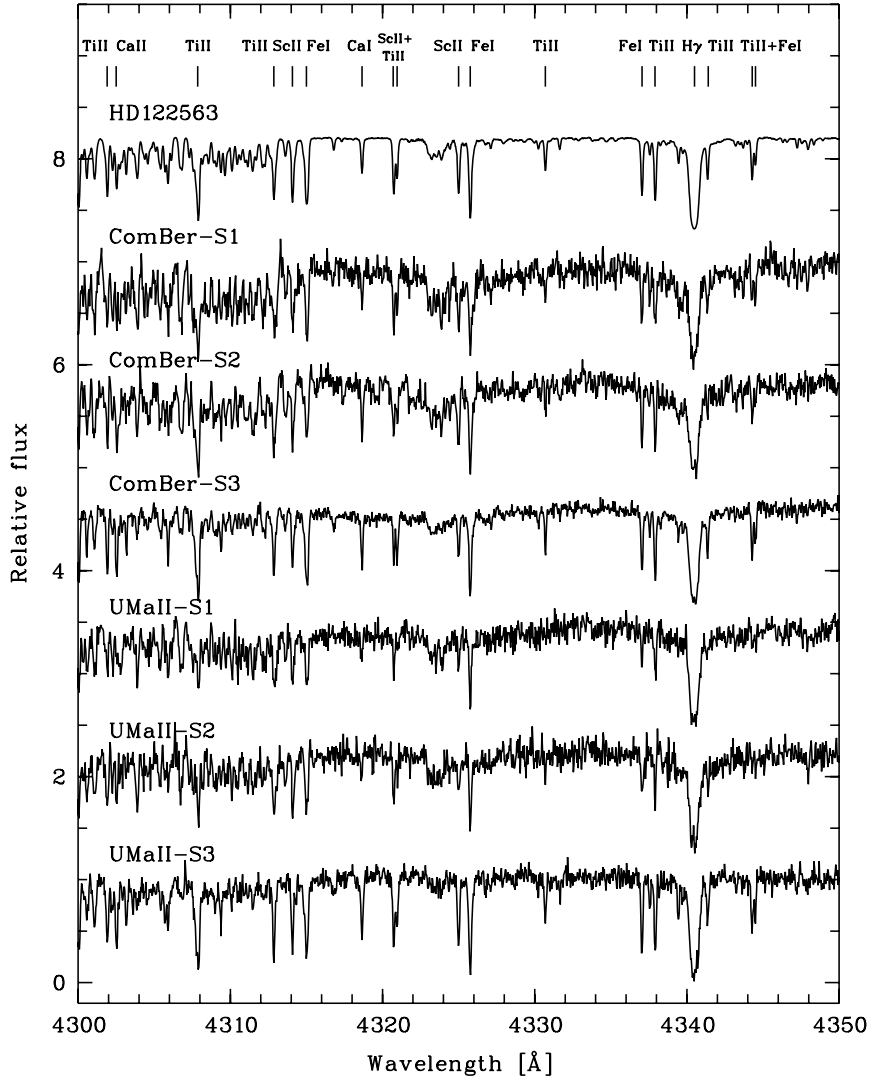

Figure 1. Keck/HIRES spectra of our program stars, shown near the $\mathrm{H} \gamma$ line at $4340 \AA$. Absorption lines are indicated. The $\mathrm{CH} G$ band is seen in all spectra blueward of the band head at $4313 \AA$.

1".15 slit, producing a spectral resolution of $R=37,000$ over the wavelength range from 4100 to $7200 \AA$ on the blue and green CCDs. The red CCD provided spectral coverage out to $8600 \AA$, but those data were compromised by second-order contamination and we do not use them in this analysis. For one star, UMa II-S3, we also used a second setting to cover bluer wavelengths from 3900 to $5300 \AA$, enabling us to obtain some additional abundances for the star (see Section 3.3.4). Finally, we observed the well-studied metal-poor halo giant HD 122563 as a comparison object. The targets, exposure times, and additional observing details are summarized in Table 2. In Figures 1 and 2, we show representative portions of the spectra of the program stars around the $\mathrm{H} \gamma$ line at $4340 \AA$ and the $\mathrm{Ba}$ line at $4554 \AA$.

The echelle data were reduced with version 2.0 of the IDL software package for HIRES developed by J. X. Prochaska and

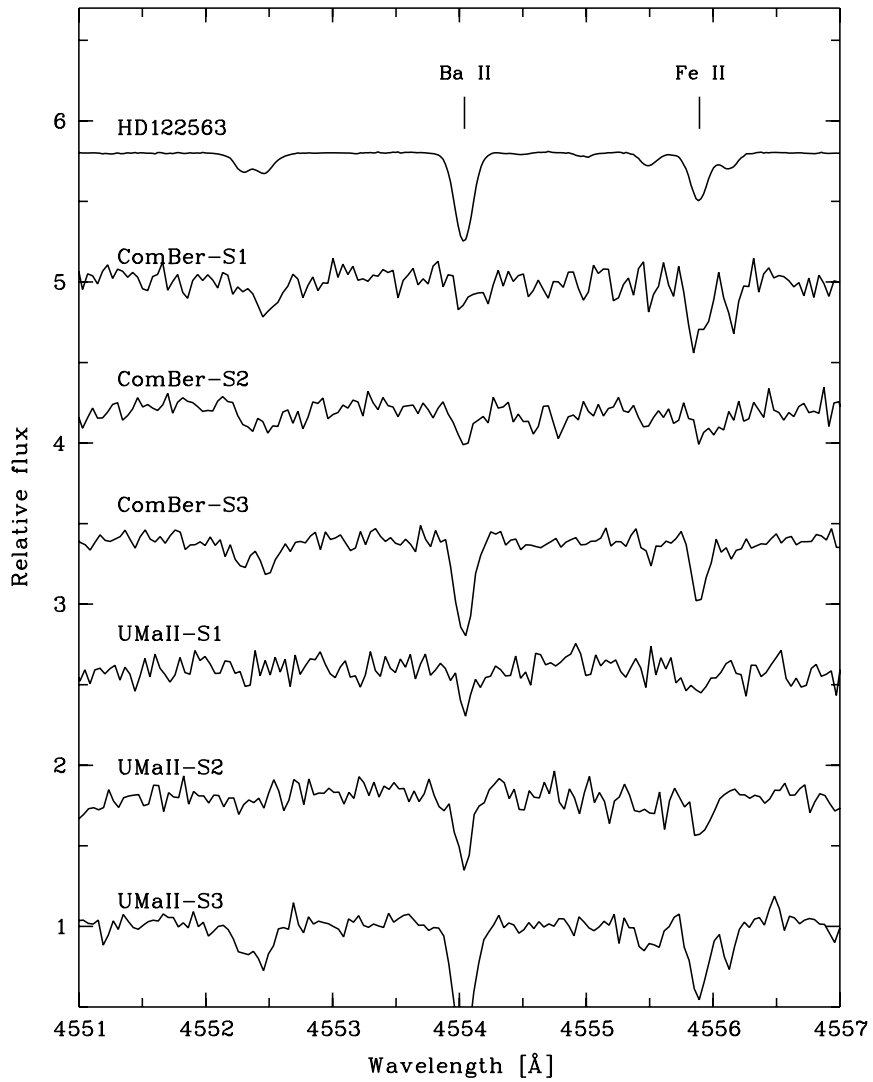

Figure 2. Spectral region around the Ba line at $4554 \AA$ for the program stars. The $\mathrm{Ba}$ and other metallic lines are detected but have significantly different line strengths in each spectrum, confirming the large spread in abundances of these elements in both UMa II and ComBer.

collaborators (R. Bernstein et al. 2010, in preparation). ${ }^{8}$ The data reduction followed standard procedures, including bias subtraction, flat-fielding, and cosmic-ray rejection. Wavelength calibration was accomplished with ThAr comparison lamp frames taken at the beginning and the end of each night. After summing the available frames for each object, the reduced and extracted spectra were normalized using a polynomial fit to the shape of each echelle order, excluding regions affected by absorption features. Finally, the overlapping echelle orders were merged together to produce the final spectrum.

Our typical integration times of several hours per star yielded signal-to-noise ratios $(\mathrm{S} / \mathrm{N})$ of $25-30$ per pixel at $5000 \AA$, which is sufficient for the detection of weak spectral features.

\footnotetext{
8 Documentation and code for this package can be found at
} http://www.ucolick.org/ xavier/HIRedux/. 
Table 3

Comparison of "Figure of Merit" of Literature Studies

\begin{tabular}{|c|c|c|c|c|c|}
\hline Study & $\mathrm{R}$ & $\begin{array}{c}\mathrm{S} / \mathrm{N} \\
\text { [per pixel] }\end{array}$ & $\begin{array}{c}\lambda \\
{[\AA]}\end{array}$ & $\mathrm{F}$ & Comment \\
\hline \multicolumn{6}{|c|}{ dSphs Studies } \\
\hline Shetrone et al. (1998) & 34000 & $24-29$ & 6300 & $130-157$ & Draco \\
\hline Shetrone et al. (2001) & 34000 & 24 & 6100 & 134 & Draco \\
\hline Shetrone et al. (2001) & 34000 & $19-36$ & 6100 & $106-210$ & Ursa Minor \\
\hline Shetrone et al. (2001) & 34000 & $13-27$ & 6100 & $72-150$ & Sextans \\
\hline Shetrone et al. (2003) & 40000 & 30 & 5800 & 207 & Sculptor, Fornax, Carina, Leo \\
\hline Bonifacio et al. (2004) & 43000 & $19-43$ & 5100 & $160-363$ & Sagittarius \\
\hline Sadakane et al. (2004) & 45000 & $50-60$ & 6100 & $368-442$ & Ursa Minor \\
\hline Geisler et al. (2005) & 16000 & 65 & 4500 & 231 & Sculptor \\
\hline Geisler et al. (2005) & 22000 & 120 & 6700 & 394 & Sculptor \\
\hline Koch et al. (2008b) & 20000 & 32 & 6500 & 98 & Hercules \\
\hline \multicolumn{6}{|c|}{ This Study } \\
\hline Ursa Major II & 34000 & $12-15$ & 4500 & $91-136$ & \\
\hline Ursa Major II & 34000 & $20-24$ & 5000 & $136-163$ & \\
\hline Ursa Major II & 34000 & $30-42$ & 6500 & $157-220$ & \\
\hline Coma Berenices & 34000 & $11-23$ & 4500 & $83-174$ & \\
\hline Coma Berenices & 34000 & $22-33$ & 5000 & $150-224$ & \\
\hline Coma Berenices & 34000 & $29-51$ & 6500 & $152-267$ & \\
\hline
\end{tabular}

To characterize the quality of the data, we use the "figure of merit" introduced by Norris et al. (2001) to compare these observations to others that have been obtained for dwarf galaxies. In Table 3, we list the figures of merit for several previous dSph observations at high resolution. These illustrate that even for stars fainter than 17th magnitude it is possible to obtain high-resolution spectra of adequate $\mathrm{S} / \mathrm{N}$ for detailed abundance studies in a few hours with the largest current telescopes.

During the course of our analysis, it became clear that one of the seven observed stars, UMa II-NM, is a foreground MW dwarf star with a similar velocity to UMa II rather than a genuine member. The spectroscopic surface gravity of $\log g=3.5$, derived from the usual Fe I-Fe II ionization balance argument, places the star at a distance of only a few kpc (the distance of UMa II is $32 \mathrm{kpc}$ ). The star sits slightly blueward of the UMa II RGB, where a star descending the asymptotic giant branch (AGB) to the horizontal branch might be located (see Figure 3), but if UMa II-NM were a member its surface gravity would be $\log g \lesssim 2$. Finally, the metallicity of UMa II-NM of $[\mathrm{Fe} / \mathrm{H}]=-1.02$ would be unusually high for UMa II, which has a mean $\mathrm{Fe}$ abundance more than 1 dex lower. We therefore discarded this star from the sample, although we do report observational details and stellar parameters for it in Tables 1 and 2 . Because this star has a velocity relatively close to the mean velocity of UMa II, removing it from the member sample does not significantly revise the systemic velocity or velocity dispersion derived by Simon \& Geha (2007).

\subsection{Line Measurements}

We use two of the strong $\mathrm{Mg}$ I $b$ lines in the green part of the HIRES spectra as well as three other $\mathrm{Mg}$ lines for our radial velocity measurements. The standard deviation of the individual line measurements is typically $<0.5 \mathrm{~km} \mathrm{~s}^{-1}$. This value increases to $0.7-1.0 \mathrm{~km} \mathrm{~s}^{-1}$ (usually depending on the $\mathrm{S} / \mathrm{N}$ level and strength of the absorption line) after all equivalent width measurements have been carried out based on the Mg linederived radial velocity correction. Consequently, the standard error is no more than $0.1 \mathrm{~km} \mathrm{~s}^{-1}$, indicating that the statistical uncertainty in our radial velocities is very small. We measure

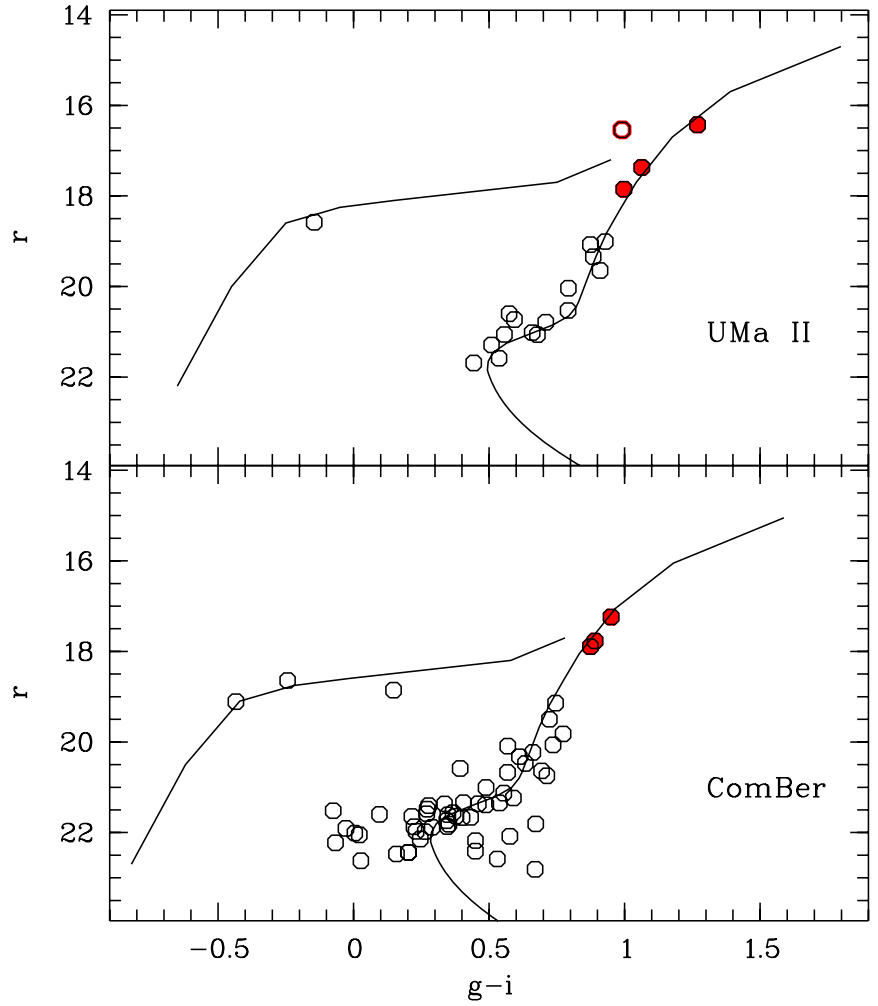

Figure 3. Updated SDSS DR7 photometry for radial velocity members of UMa II (top panel) and ComBer (bottom panel) from Simon \& Geha (2007). Also shown are the isochrone of M92 and the horizontal branch of M13 (solid lines), both corrected for Galactic extinction and shifted to distances of $32 \mathrm{kpc}$ and $44 \mathrm{kpc}$ for the two dwarf galaxies (data from Clem 2006). Our highresolution targets are shown with red solid circles. The red open circle refers to UMa II-NM. As can be seen, this star sits slightly off the giant branch track.

(A color version of this figure is available in the online journal.)

the velocity of HD 122563 to verify that our measurements are on the correct velocity scale. We find a velocity of $-25.1 \pm$ $0.2 \mathrm{~km} \mathrm{~s}^{-1}$ for the star, in reasonable agreement with the velocity determined by Aoki et al. (2007b) of $-26.0 \pm 0.2 \mathrm{~km} \mathrm{~s}^{-1}$, as well as other literature values. The HIRES radial velocities are 
Table 4

Equivalent Width Measurements

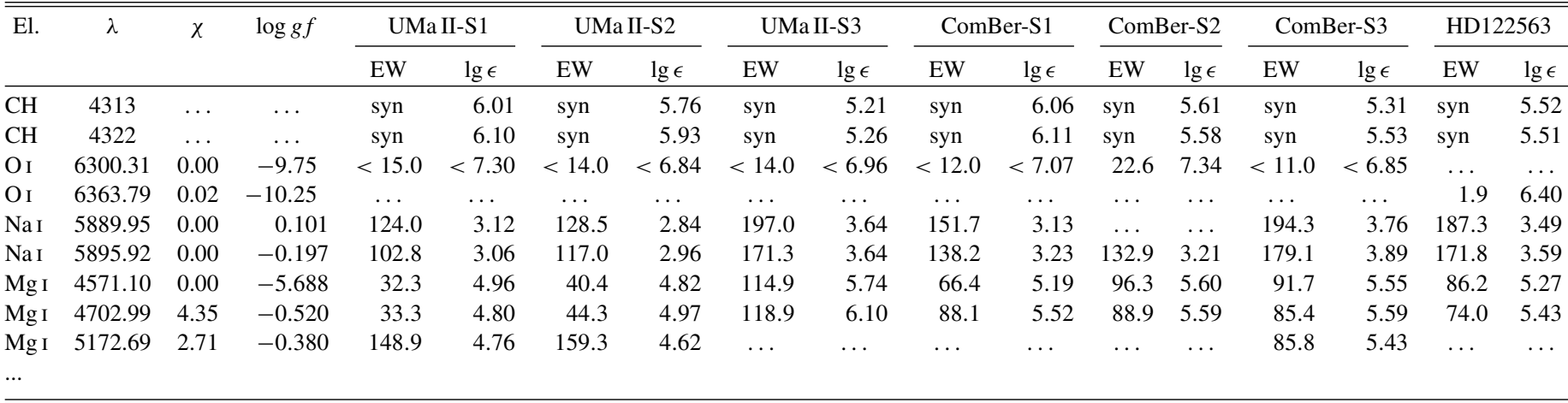

(This table is available in its entirety in a machine-readable form in the online journal. A portion is shown here for guidance regarding its form and content.)

slightly offset (1-3 $\mathrm{km} \mathrm{s}^{-1}$ ) from those obtained by Simon \& Geha (2007) from DEIMOS $R \sim 6000$ spectra. We speculate that this offset results from the DEIMOS velocity zero point determined by Simon \& Geha (2007), which was tied to a different set of stars, and hence should not indicate any problems with the HIRES measurements.

For the measurements of atomic absorption lines we employ a line list based on the compilations of Aoki et al. (2007b) and Ivans et al. (2006). We added the newly determined Fe II $g f$ values of Meléndez \& Barbuy (2009). The molecular line data employed for CH were provided by B. Plez (B. Plez et al. 2009, in preparation; the latest version of the list is described in Plez et al. 2008, and some basic details are given in Hill et al. 2002). Hyperfine-structure (HFS) data for Sc and Mn were taken from the Kurucz compilation (Kurucz 1998). ${ }^{9}$

In Table 4, we list the lines used and their measured equivalent widths. Only line measurements with reduced equivalent widths $\log (\mathrm{EW} / \lambda)<-4.5$ were employed in the abundance analysis of each star. The $\mathrm{Mg} b$ lines were thus excluded in several cases since these lines are too strong and fall in the flat part of the curve of growth. While we did measure the $\mathrm{Mg} b$ triplet and other lines, if they were not used in the analysis they are not listed in Table 4 . We verify our equivalent width measurement techniques by comparing the results for HD 122563 with the study of Aoki et al. (2007b), which covered a similar wavelength range. Figure 4 illustrates the excellent agreement between the two data sets. For blended lines, lines with HFS, and molecular features, we use a spectral synthesis approach. The abundance of a given species is obtained by matching a synthetic spectrum of known abundance to the observed spectrum.

Since the average $\mathrm{S} / \mathrm{N}$ of the data is modest, we also calculate what the minimum detectable equivalent width is for our data. We chose one example star, UMa II-S1, which has among the lowest $\mathrm{S} / \mathrm{N}$ in the sample, has the highest temperature, and the second lowest metallicity, which means that the lines are very weak. Quantifying the minimum level in this star thus serves as rather conservative estimate for the entire sample. Using the formula given in Norris et al. (2001), we estimate the approximate $3 \sigma$ detectable equivalent width to be $\sim 22 \mathrm{~m} \AA$ for the blue and $15 \mathrm{~m} \AA$ for the red part of the data ( $2 \sigma$ values are $\sim 17 \mathrm{~m} \AA$ and $10 \mathrm{~m} \AA$, respectively).

A number of lines fall in the range between $2 \sigma$ and $3 \sigma$ for this star. However, since our equivalent width measurements have significant uncertainties from the continuum placement,

\footnotetext{
9 http://kurucz.harvard.edu/
}

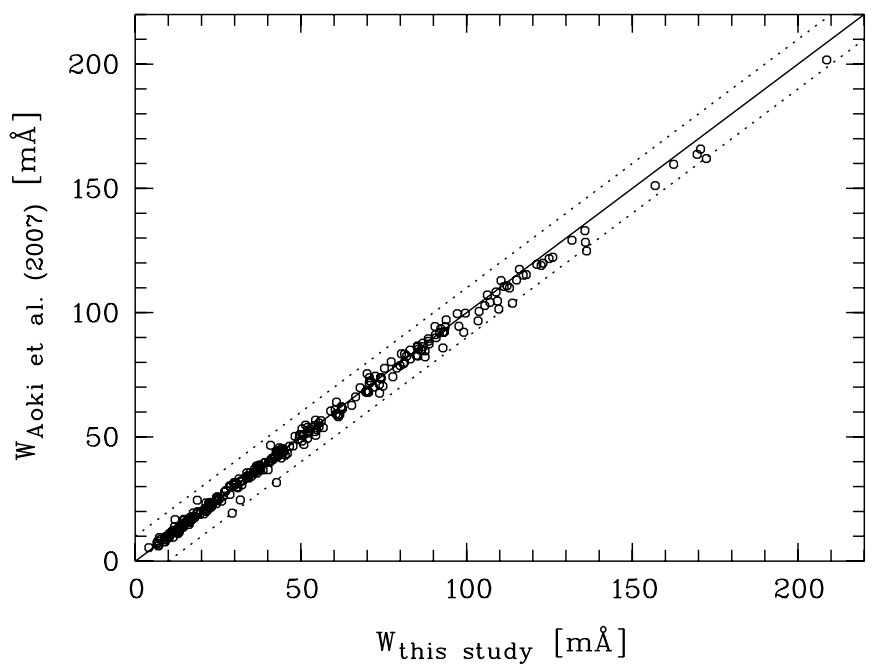

Figure 4. Comparison of the equivalent width measurements in our HIRES spectrum of HD 122563 with those listed in Aoki et al. (2007b). The solid line indicates one-to-one correspondence, the dotted lines represent deviations of $\pm 10 \mathrm{~m} \AA$ from equality to guide the eye.

we do not discard these lines. Another reason for keeping these (mostly $\mathrm{Fe}$ ) lines is that weak lines near the detection limit are needed for the determination of the microturbulent velocity. Only five measured lines in UMa II-S1 (of Ti I, Ti II, Fe I, and two $\mathrm{Ni}$ I) have equivalent widths less than $2 \sigma$ according to the Norris et al. (2001) criterion (none below $1 \sigma$ ), but we emphasize that in a strict statistical sense, all of these features are detected at the $3 \sigma$ level or higher. These lines are, however, found to generally yield abundances in good agreement with the stronger lines. In the case of $\mathrm{Ni}$, only three lines could be measured in the star and they are all very weak. Two of them have a $1.5 \sigma$ detection, while the third one is only at the $2.5 \sigma$ level. In the absence of stronger Ni lines, we keep the present measurements but assign a nominal uncertainty of 0.40 dex (based on consideration of the measurement uncertainties). Regarding the other stars, the situation is less severe since the $\mathrm{S} / \mathrm{N}$ of the data is generally better, the lines are stronger, and more lines are available. The exception is UMa II-S2 for which we have no detected Ni lines, and therefore only an upper limit for the $\mathrm{Ni}$ abundance.

Upper limits on abundances of elements for which no lines were detected can provide useful additional information for the interpretation of the overall abundance patterns, and the possible origins of the stars of interest. Based on the $\mathrm{S} / \mathrm{N}$ in the spectral region of the line, and employing the formula given in Frebel 


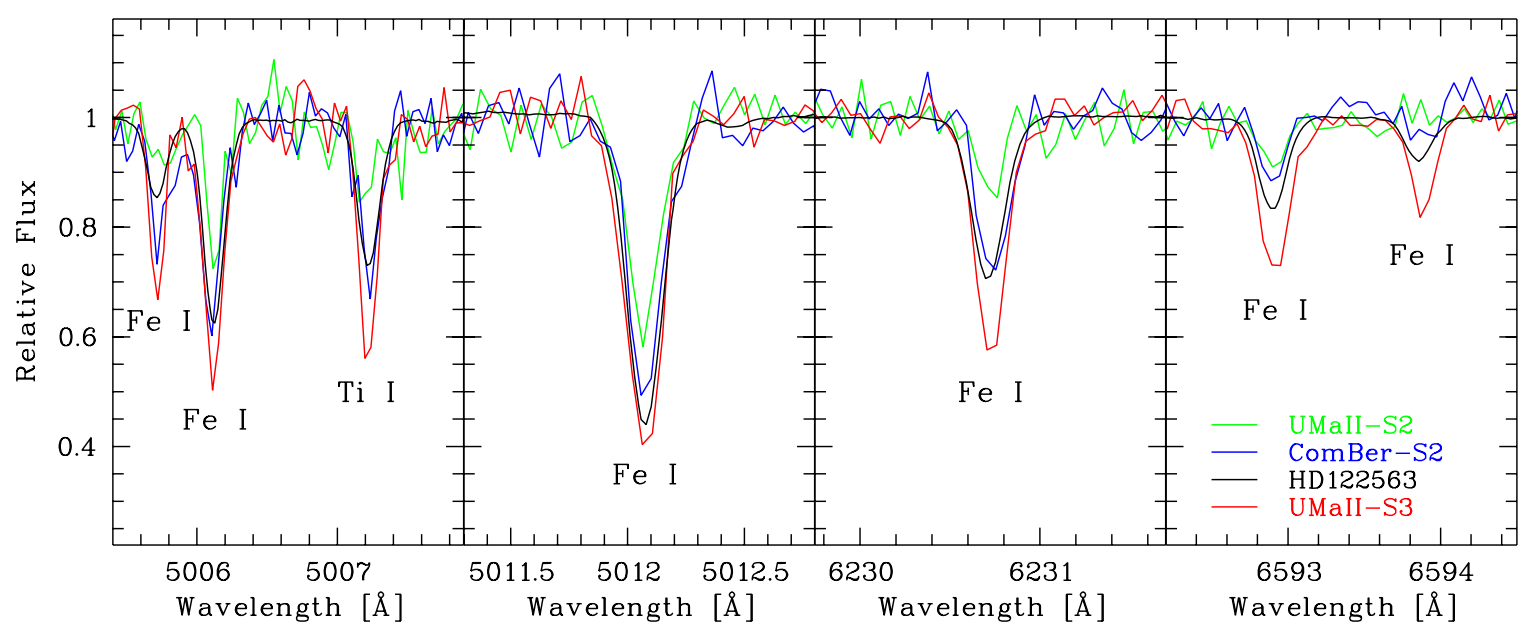

Figure 5. Several $\mathrm{Fe}$ I lines of three stars with similar temperatures to illustrate $\mathrm{Fe}$ abundance differences. UMa II-S2 (green) with $[\mathrm{Fe} / \mathrm{H}]=-3.2, \mathrm{ComBer}-\mathrm{S} 2$ (blue) with $[\mathrm{Fe} / \mathrm{H}]=-2.9$, and UMa II-S3 with $[\mathrm{Fe} / \mathrm{H}]=-2.3$ (red). The black line refers to HD 122563, the MW halo star with $[\mathrm{Fe} / \mathrm{H}]=-2.8$.

(A color version of this figure is available in the online journal.)

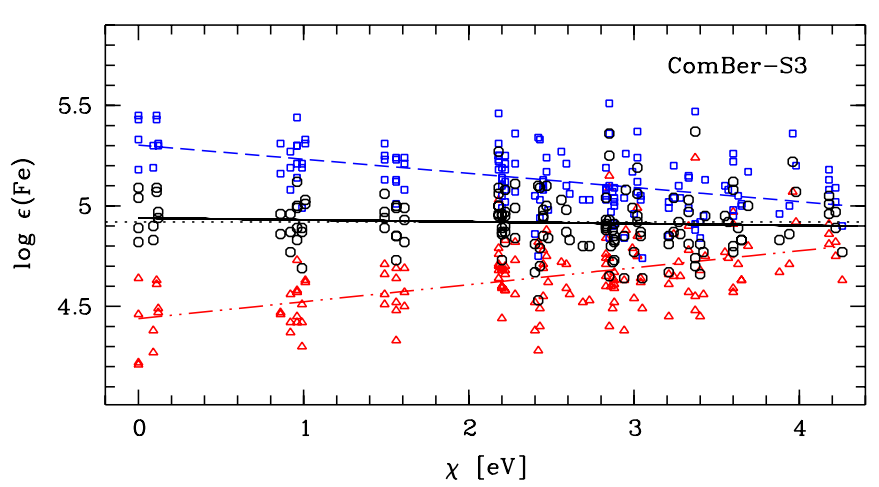

Figure 6. Fe I abundances as a function of excitation potential, $\chi$, in ComBer-S3 as an example. Three different temperatures are shown: $4800 \mathrm{~K}$ (blue squares), $4600 \mathrm{~K}$ (black open circles), $4400 \mathrm{~K}$ (red triangles). The dotted line indicates the mean abundance of all Fe lines for the adopted temperature of $4600 \mathrm{~K}$. The dashed/solid/dot-dashed lines shows the corresponding fits to the data sets.

(A color version of this figure is available in the online journal.)

et al. (2006a), we derive $3 \sigma$ upper limits for several elements. In Table 4, we list upper limits of a given element for whichever line produced the tightest upper limit.

\subsection{Stellar Parameters}

In Figure 5, we show pieces of spectra containing a number of Fe I lines for three dwarf galaxy stars and HD 122563. All of the stars have similar temperatures and thus allow for a simple, visual comparison of the Fe I line strengths. This quickly illustrates the different metallicities sampled by our program stars. It is clear that UMa II-S2 has much weaker Fe lines than the other stars, demonstrating even without any analysis that this star must be more metal-poor than HD 122563 (at $[\mathrm{Fe} / \mathrm{H}]=-2.8$ ).

\subsubsection{Effective Temperature}

We then derive spectroscopic effective temperatures by demanding that there be no trend of abundances with excitation potential for the Fe I lines. As an example, Figure 6 shows Fe I abundances as a function of excitation potential based on our spectroscopically derived value for ComBer-S3. We also show $\mathrm{Fe}$ abundances for temperatures of $\pm 200 \mathrm{~K}$ to illustrate the sensitivity of the method to the assumed temperature. By varying the temperature and comparing the derived trends to zero given the statistical uncertainty on the slope, we determine the effective temperature and its uncertainty. As illustrated in Figure 6, a $200 \mathrm{~K}$ change in temperature causes a strong trend in the abundances as a function of excitation potential. Generally, at $3 \sigma$ confidence, we are able to determine the temperature to within $\sim 150 \mathrm{~K}$ using this technique.

The advantage of this approach over photometric temperatures is that it is reddening-free and independent of the empirical calibrations that are needed to convert stellar colors into effective temperatures. Nevertheless, for completeness, we calculated photometric temperatures from various ugriz colors by using the Yonsei-Yale isochrones (Kim et al. 2002) and the color tables of Castelli (http://wwwuser.oat.ts.astro.it/castelli/). The differences between the temperatures obtained from the different colors vary between $\sim 150$ and more than $300 \mathrm{~K}$ for a given metallicity. While the average temperatures agree well with our spectroscopic values (within $100 \mathrm{~K}$ ) for some stars, most of them agree to within $250 \mathrm{~K}$. The spectroscopically derived temperatures are lower than photometrically derived ones. Kirby et al. (2008) also calculated photometric temperatures (also using the Yonsei-Yale isochrone, but with different color tables; E. Kirby 2008, private communication) and in most cases, our spectroscopic values agree with their photometric values within $200 \mathrm{~K}$. Systematic uncertainties regarding the determination method of temperature can be estimated to be $\sim 200 \mathrm{~K}$.

\subsubsection{Surface Gravity}

Using the ionization balance, i.e., demanding that Fe I lines yield the same abundance as Fe II lines, we derive the surface gravity, $\log g$, for all of the stars. Based on the standard deviations of the averaged Fe I and Fe II abundances $(\sim 0.15-$ $0.25 \mathrm{dex}$ ), we estimate an uncertainty of $0.3 \mathrm{dex}$ in $\log g$. The micro-turbulence, $v_{\text {micr }}$, is obtained iteratively in this process by demanding no trend of abundances with equivalent widths. Uncertainties in this parameter are estimated to be $0.3 \mathrm{~km} \mathrm{~s}^{-1}$. Table 5 lists the individual stellar parameters. Figure 7 shows the adopted stellar parameters of our program stars in comparison with $\alpha$-enhanced $([\alpha / \mathrm{Fe}]=0.4) 12$ Gyr isochrones (Green et al. 1984; Kim et al. 2002) covering a range of metallicities. Our values generally agree very well with those of the isochrone. This also shows that our Fe line abundances are probably not significantly affected by non-LTE effects. 


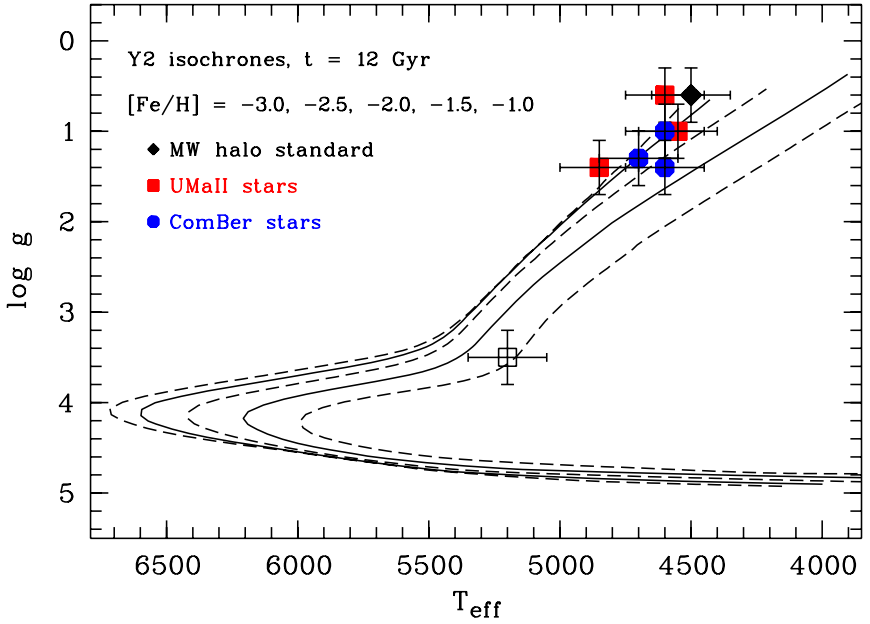

Figure 7. Adopted stellar parameters in comparison with $12 \mathrm{Gyr}$ isochrones with $[\alpha / \mathrm{Fe}]=0.4$ and metallicity ranging from $[\mathrm{Fe} / \mathrm{H}]=-1$ to $-3($ Green et al. 1984; Kim et al. 2002). The more metal-rich isochrones are shifted toward lower temperatures at a given surface gravity. Red squares indicate UMa II stars; blue circles are ComBer stars. The open square shows the observed star that turned out to be a non-member of UMa II. The MW standard star HD 122563 is marked with a black diamond.

(A color version of this figure is available in the online journal.)

Table 5

Stellar Parameters

\begin{tabular}{lcccc}
\hline \hline \multicolumn{1}{c}{ Star } & $\begin{array}{c}T_{\text {eff }} \\
{[\mathrm{K}]}\end{array}$ & $\begin{array}{c}\log (g) \\
{[\mathrm{dex}]}\end{array}$ & $\begin{array}{c}{[\mathrm{Fe} / \mathrm{H}]} \\
{[\mathrm{dex}]}\end{array}$ & $\begin{array}{c}v_{\text {micr }} \\
{\left[\mathrm{km} \mathrm{s}^{-1}\right]}\end{array}$ \\
\hline UMa II-S1 & 4850 & 1.4 & -3.10 & 2.0 \\
UMa II-NM & 5200 & 3.5 & -1.02 & 1.6 \\
UMa II-S2 & 4600 & 0.6 & -3.23 & 2.5 \\
UMa II-S3 & 4550 & 1.0 & -2.34 & 2.2 \\
ComBer-S1 & 4700 & 1.3 & -2.31 & 2.5 \\
ComBer-S2 & 4600 & 1.4 & -2.88 & 2.0 \\
ComBer-S3 & 4600 & 1.0 & -2.53 & 2.2 \\
HD 122563 & 4500 & 0.6 & -2.77 & 2.5 \\
\hline
\end{tabular}

Note. Temperatures are rounded to the nearest $10 \mathrm{~K}$.

\subsection{Model Atmospheres}

Our abundance analysis utilizes one-dimensional planeparallel Kurucz model atmospheres with no overshooting (Kurucz 1993). They are computed under the assumption of local thermodynamic equilibrium (LTE). We use the 2002 version of the MOOG synthesis code (Sneden 1973) for this analysis. Scattering in MOOG is currently treated as true absorption. The missing implementation of a source function that sums both absorption and scattering components (rather than treating continuous scattering as true absorption) will be incorporated in future MOOG versions (J. Sobeck et al. 2010, in preparation).

In order to arrive at our final abundance ratios $[\mathrm{X} / \mathrm{Fe}]$, which are given with respect to the solar values, we employ the Asplund et al. (2005) solar abundances. The elemental abundances for all of the target stars are given in Tables 6 and 7.

\subsection{Uncertainties}

\subsubsection{Measurement and Stellar Parameter Uncertainties}

To assess the level of measurement uncertainties, we made use of the fact that a number of absorption lines fall at the end of a given order and are thus measurable twice in the two consecutive orders. By comparing both sets of line measurements, we robustly estimate that the average total uncertainty on our
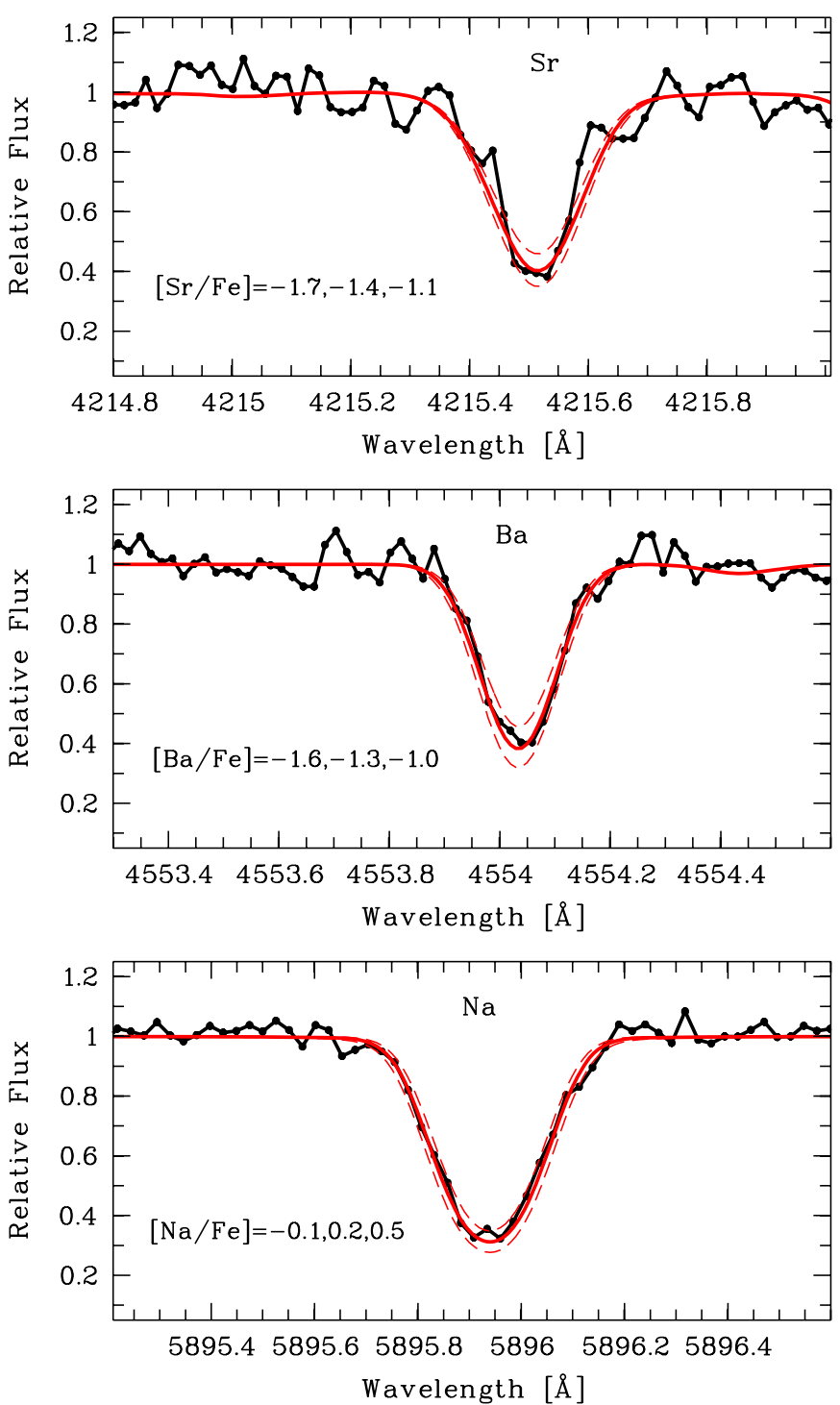

Figure 8. Examples of strong lines $(\mathrm{Sr}, \mathrm{Ba}, \mathrm{Na})$ used in the analysis of ComBer-S3, the star with the strongest Ba line (see Figure 2). Black lines are the data; red lines refer to synthetic spectra of given abundances. The synthetic spectra demonstrate that even for lines beyond the linear part of the curve of growth, abundances are measurable to within the $\sim 0.3$ dex or better.

(A color version of this figure is available in the online journal.)

equivalent width measurements is $14 \mathrm{~m} \AA$ for the blue lines and $9 \mathrm{~m} \AA$ for the red lines (where the division between blue and red for this purpose is $\sim 5700 \AA$ ). These uncertainties translate into abundance uncertainties of $\sim 0.20 \mathrm{dex}$. This is in good agreement with the standard deviations of the abundances derived from individual lines of a given element (as long as the number of lines is more than just a few), and can thus be regarded as a general, robust estimate of the continuum placement uncertainty ("random uncertainty") in our derived abundances.

In Figure 8, we show the sensitivity of the abundances derived from strong lines near the flat part of the curve-of-growth. From this, we estimate the measurement uncertainties of $\mathrm{Sr}, \mathrm{Ba}$, and $\mathrm{Na}$ abundances to be $0.3 \mathrm{dex}$. This is in agreement with what is found from the continuum placement uncertainties and should account for the line strengths.

For abundances of elements represented by only one line (other than $\mathrm{Sr}, \mathrm{Ba}$, and $\mathrm{Na}$ ), we adopt a formal uncertainty of $0.20 \mathrm{dex}$, based on the measurement uncertainties investigated 
Table 6

HIRES Abundances of the UMa II Stars

\begin{tabular}{|c|c|c|c|c|c|c|c|c|c|c|c|c|c|c|c|}
\hline \multirow[t]{2}{*}{ Species } & \multicolumn{5}{|c|}{ UMa II-S1 } & \multicolumn{5}{|c|}{ UMa II-S2 } & \multicolumn{5}{|c|}{ UMa II-S3 } \\
\hline & $\lg \epsilon(\mathrm{X})$ & {$[\mathrm{X} / \mathrm{H}]$} & {$[\mathrm{X} / \mathrm{Fe}]$} & $N$ & $\sigma$ & $\lg \in(\mathrm{X})$ & {$[\mathrm{X} / \mathrm{H}]$} & {$[\mathrm{X} / \mathrm{Fe}]$} & $N$ & $\sigma$ & $\lg \epsilon(\mathrm{X})$ & {$[\mathrm{X} / \mathrm{H}]$} & {$[\mathrm{X} / \mathrm{Fe}]$} & $N$ & $\sigma$ \\
\hline $\mathrm{C}$ & 6.06 & -2.33 & 0.77 & 2 & 0.20 & 5.70 & -2.69 & 0.54 & 2 & 0.20 & 5.24 & -3.15 & -0.81 & 2 & 0.20 \\
\hline $\mathrm{O}_{\mathrm{I}}$ & $<7.30$ & $<-1.36$ & $<1.74$ & 1 & $\ldots$ & $<7.84$ & $<-0.82$ & $<2.41$ & 1 & $\ldots$ & $<6.96$ & $<-1.70$ & $<0.64$ & 1 & $\ldots$ \\
\hline $\mathrm{NaI}$ & 3.06 & -3.11 & -0.01 & 2 & 0.30 & 2.93 & -3.24 & -0.01 & 2 & 0.30 & 3.68 & -2.49 & -0.15 & 2 & 0.30 \\
\hline $\mathrm{Mg}_{\mathrm{I}}$ & 4.87 & -2.66 & 0.44 & 5 & 0.11 & 4.77 & -2.76 & 0.47 & 5 & 0.15 & 5.95 & -1.58 & 0.76 & 4 & 0.17 \\
\hline $\mathrm{Al} \mathrm{I}$ & $<5.23$ & $<-1.14$ & $<1.96$ & 1 & $\ldots$ & $<5.25$ & $<-1.12$ & $<2.11$ & 1 & $\ldots$ & 3.70 & -2.66 & -0.34 & 1 & 0.30 \\
\hline Si I & $<5.77$ & $<-1.74$ & $<1.36$ & 1 & $\ldots$ & $<5.74$ & $<-1.77$ & $<1.46$ & 1 & $\ldots$ & 6.08 & -1.43 & 0.91 & 1 & 0.30 \\
\hline $\mathrm{Ca} \mathrm{I}$ & 3.41 & -2.90 & 0.20 & 9 & 0.30 & 3.46 & -2.85 & 0.38 & 12 & 0.19 & 4.50 & -1.81 & 0.53 & 22 & 0.17 \\
\hline Sc II & 0.20 & -2.85 & 0.25 & 3 & 0.18 & -0.30 & -3.35 & -0.12 & 2 & 0.18 & 0.67 & -2.38 & -0.04 & 5 & 0.15 \\
\hline Ti I & 2.07 & -2.83 & 0.27 & 6 & 0.24 & 1.93 & -2.97 & 0.26 & 5 & 0.24 & 2.60 & -2.30 & 0.04 & 21 & 0.10 \\
\hline Ti II & 2.02 & -2.88 & 0.22 & 15 & 0.18 & 1.80 & -3.10 & 0.13 & 16 & 0.23 & 2.77 & -2.13 & 0.21 & 27 & 0.20 \\
\hline$V_{\text {I }}$ & $<1.58$ & $<-2.42$ & $<0.68$ & 1 & $\ldots$ & $<1.37$ & $<-2.63$ & $<0.60$ & 1 & $\ldots$ & 1.73 & -2.27 & 0.07 & 2 & 0.10 \\
\hline Cr I & 2.28 & -3.36 & -0.26 & 4 & 0.09 & 1.91 & -3.73 & -0.50 & 3 & 0.25 & 2.98 & -2.66 & -0.32 & 11 & 0.13 \\
\hline Mn I & 2.27 & -3.12 & -0.02 & 2 & 0.10 & $<2.40$ & $<-2.99$ & $<0.24$ & 1 & $\ldots$ & 2.48 & -2.91 & -0.57 & 3 & 0.10 \\
\hline $\mathrm{Fe} I$ & 4.35 & -3.10 & $\ldots$ & 74 & 0.16 & 4.22 & -3.23 & $\ldots$ & 64 & 0.16 & 5.11 & -2.34 & $\ldots$ & 133 & 0.15 \\
\hline Fe II & 4.35 & -3.10 & 0.00 & 7 & 0.13 & 4.28 & -3.17 & 0.06 & 6 & 0.23 & 5.11 & -2.34 & 0.00 & 19 & 0.16 \\
\hline Co I & $<2.77$ & $<-2.15$ & $<0.95$ & 1 & $\ldots$ & $<2.59$ & $<-2.33$ & $<0.90$ & 1 & $\ldots$ & 2.49 & -2.43 & -0.09 & 4 & 0.12 \\
\hline Ni I & 3.44 & -2.79 & 0.31 & 3 & 0.40 & $<3.58$ & $<-2.65$ & $<0.58$ & 1 & $\ldots$ & 3.87 & -2.36 & -0.02 & 10 & 0.16 \\
\hline $\mathrm{Cu} \mathrm{I}$ & $<1.84$ & $<-2.37$ & $<0.73$ & 1 & $\ldots$ & $<1.60$ & $<-2.61$ & $<0.62$ & 1 & $\ldots$ & $<1.42$ & $<-2.79$ & $<-0.45$ & 1 & $\ldots$ \\
\hline $\mathrm{Zn}$ I & $2.35^{\mathrm{a}}$ & -2.25 & 0.85 & 1 & 0.20 & 1.42 & -3.18 & 0.05 & 1 & 0.20 & 2.26 & -2.34 & 0.00 & 1 & 0.20 \\
\hline Sr II & -0.90 & -3.82 & -0.72 & 1 & 0.30 & -1.30 & -4.22 & -0.99 & 1 & 0.30 & -0.90 & -3.82 & -1.48 & 1 & 0.30 \\
\hline Y II & $<-1.15$ & $<-3.36$ & $<-0.26$ & 1 & $\ldots$ & $<-1.27$ & $<-3.48$ & $<-0.25$ & 1 & $\ldots$ & -1.27 & -3.48 & -1.14 & 1 & 0.15 \\
\hline Zr II & $<-0.19$ & $<2.78$ & $<0.32$ & 1 & $\ldots$ & $<-0.43$ & $<-3.02$ & $<0.21$ & 1 & $\ldots$ & -0.35 & -2.94 & -0.60 & 1 & 0.30 \\
\hline Ba II & -2.27 & -4.44 & -1.34 & 1 & 0.30 & -2.20 & -4.37 & -1.14 & 2 & 0.30 & -0.73 & -2.90 & -0.56 & 3 & 0.30 \\
\hline La II & $<-1.54$ & $<-2.67$ & $<0.43$ & 1 & $\ldots$ & $<-1.85$ & $<-2.98$ & $<0.25$ & 1 & $\ldots$ & -1.49 & -2.62 & -0.28 & 3 & 0.40 \\
\hline Ce II & $<-1.13$ & $<-2.71$ & $<0.39$ & 1 & $\ldots$ & $<-1.49$ & $<-3.07$ & $<0.16$ & 1 & $\ldots$ & $<-1.28$ & $<-2.86$ & $<-0.52$ & 1 & $\ldots$ \\
\hline $\mathrm{Nd}$ II & $<-0.95$ & $<-2.40$ & $<0.70$ & 1 & $\ldots$ & $<-1.21$ & $<-2.66$ & $<0.57$ & 1 & $\ldots$ & $<-1.43$ & $<-2.88$ & $<-0.54$ & 1 & $\ldots$ \\
\hline Sm II & $<-0.82$ & $<-1.83$ & $<1.27$ & 1 & $\ldots$ & $<-1.53$ & $<-2.54$ & $<0.69$ & 1 & $\ldots$ & $<-1.79$ & $<-2.80$ & $<-0.46$ & 1 & $\ldots$ \\
\hline Eu II & $<-2.00$ & $<-2.52$ & $<0.58$ & 1 & $\ldots$ & $<-2.20$ & $<-2.72$ & $<0.51$ & 1 & $\ldots$ & $<-2.20$ & $<-2.72$ & $<-0.38$ & 1 & $\ldots$ \\
\hline
\end{tabular}

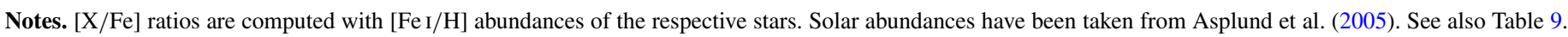
For abundances measured from only one line, we adopt a nominal uncertainty of 0.20 dex.

a The $\mathrm{Zn}$ lines are somewhat distorted; this may lead to an overestimated $\mathrm{Zn}$ abundance

above (these values do not apply to HD122563 because of the higher $\mathrm{S} / \mathrm{N}$ level). Despite having two measurements for $\mathrm{C}$, we also adopt 0.20 dex for this element since the continuum placement can be difficult for molecular bands. For many elements where only few lines are measured the standard deviations are unrealistically small $(<0.10$ dex $)$ compared with our finding regarding the general measurement uncertainties. We thus adopt a minimum uncertainty of 0.10 dex in such cases.

We tested the robustness of our derived abundances by changing one stellar parameter at a time by an amount approximately equal to its random uncertainty. In Table 8 , we give a summary of these individual sources of error as well as a total uncertainty for each element of an example star, ComBer-S3. Taking all the sources of errors into account, the abundances derived from atomic lines have an average uncertainty of $\sim 0.25$ dex.

\subsubsection{Uncertainties from Different Log $g f$ Values}

In Section 3, our primary comparison sample will be that of Cayrel et al. (2004) and François et al. (2007), which uses slightly different atomic data than we do. To determine whether our particular choice of $\log g f$ values (as adopted from Aoki et al. 2007b; Section 2.2) results in systematic abundance offsets, we compare our values to those of Cayrel et al. We find small $\log g f$ differences for most elements. When more than just a few lines are measured for each element, we estimate a constant offset for all stars based on the number of lines in common between our list and that of Cayrel et al. For $\mathrm{Mg}$ I, we estimate the required offset to be $-0.11 \mathrm{dex}$, for $\mathrm{Ca} \mathrm{I}+0.05 \mathrm{dex}$, for
Ti I +0.05 dex, for Ti II -0.09 dex, for Sc II +0.09 dex, and for Zn I -0.04 dex.

We find no significant systematic offset for Fe I (very few individual lines have larger $g f$ differences but those are averaged out in the final abundances), $\mathrm{Na}, \mathrm{Cr}$, and $\mathrm{Mn}$. No lines in common are found for $\mathrm{Al}, \mathrm{Si}$, and $\mathrm{Ni}$, so we cannot derive an offset. We then compare the abundances corrected for the $\log g f$ differences as listed above. We caution, however, that the offsets applied are based only on the subsets of lines in common and do not reflect a more detailed comparison of the employed $g f$ values themselves. We also note for completeness that we recalculated the Cayrel et al. relative abundances with the same solar abundances employed here (Asplund et al. 2005).

\subsubsection{Model Atmosphere Uncertainties}

Systematic uncertainties arising from the choice of model atmospheres may add to the error budget. To test the effect of our choice of model atmospheres, we ran a differential abundance analysis for the star ComBer-S3 by employing a Kurucz model and the MOOG model atmosphere code as well as a MARCS model (Gustafsson et al. 2008) and a corresponding code (Uppsala LTE spectrum synthesis code "BSYN," ver. 7.05).

Using the BSYN code, we are able to choose whether a more proper scattering treatment (than what is used in MOOG) is "switched on." This helps in quantifying various effects associated with the different model atmospheres. We 
Table 7

HIRES Abundances of the ComBer Stars

\begin{tabular}{|c|c|c|c|c|c|c|c|c|c|c|c|c|c|c|c|}
\hline \multirow[b]{2}{*}{ Species } & \multicolumn{5}{|c|}{ ComBer-S1 } & \multicolumn{5}{|c|}{ ComBer-S2 } & \multicolumn{5}{|c|}{ ComBer-S3 } \\
\hline & $\lg \epsilon(\mathrm{X})$ & {$[\mathrm{X} / \mathrm{H}]$} & {$[\mathrm{X} / \mathrm{Fe}]$} & $N$ & $\sigma$ & $\lg \epsilon(\mathrm{X})$ & {$[\mathrm{X} / \mathrm{H}]$} & {$[\mathrm{X} / \mathrm{Fe}]$} & $N$ & $\sigma$ & $\lg \epsilon(\mathrm{X})$ & {$[\mathrm{X} / \mathrm{H}]$} & {$[\mathrm{X} / \mathrm{Fe}]$} & $N$ & $\sigma$ \\
\hline $\mathrm{C}$ & 6.09 & -2.30 & 0.01 & 2 & 0.20 & 5.60 & -2.79 & 0.09 & 2 & 0.20 & 5.42 & -2.97 & -0.44 & 2 & 0.20 \\
\hline OI & $<7.07$ & $<-1.59$ & $<0.72$ & 1 & $\ldots$ & $<7.34$ & $<-1.32$ & $<1.56$ & 1 & $\ldots$ & $<6.85$ & $<-1.81$ & $<0.72$ & 1 & $\cdots$ \\
\hline $\mathrm{NaI}$ & 3.12 & -3.05 & -0.74 & 2 & 0.30 & 3.13 & -3.04 & -0.16 & 1 & 0.30 & 3.83 & -2.34 & 0.19 & 2 & 0.30 \\
\hline $\mathrm{Mg}_{\mathrm{I}}$ & 5.38 & -2.15 & 0.16 & 3 & 0.20 & 5.63 & -1.90 & 0.98 & 3 & 0.10 & 5.50 & -2.03 & 0.50 & 4 & 0.10 \\
\hline Al I & $<5.25$ & $<-1.12$ & $<1.19$ & 1 & $\ldots$ & $<5.15$ & $<-1.22$ & $<1.66$ & 1 & $\ldots$ & $<5.06$ & $<-1.31$ & $<1.22$ & 1 & $\ldots$ \\
\hline Si I & $<5.74$ & $<-1.77$ & $<0.54$ & 1 & $\ldots$ & $<5.71$ & $<-1.80$ & $<1.08$ & 1 & $\ldots$ & $<5.48$ & $<-2.03$ & $<0.50$ & 1 & $\ldots$ \\
\hline Ca I & 4.11 & -2.20 & 0.11 & 18 & 0.18 & 4.06 & -2.25 & 0.63 & 17 & 0.21 & 4.38 & -1.93 & 0.60 & 22 & 0.15 \\
\hline Sc II & 0.32 & -2.73 & -0.42 & 5 & 0.26 & 0.72 & -2.33 & 0.55 & 4 & 0.19 & -0.05 & -3.10 & -0.57 & 4 & 0.10 \\
\hline Ti I & 2.41 & -2.49 & -0.18 & 12 & 0.18 & 2.35 & -2.55 & 0.33 & 13 & 0.22 & 2.54 & -2.36 & 0.17 & 23 & 0.12 \\
\hline Ti II & 2.58 & -2.32 & -0.01 & 27 & 0.14 & 2.42 & -2.48 & 0.40 & 21 & 0.21 & 2.66 & -2.24 & 0.29 & 30 & 0.16 \\
\hline $\mathrm{V}_{\mathrm{I}}$ & $<1.18$ & $<-2.82$ & $<-0.51$ & 1 & $\ldots$ & $<1.46$ & $<-2.54$ & $<0.34$ & 1 & $\ldots$ & $<1.10$ & $<-2.90$ & $<-0.37$ & 1 & $\ldots$ \\
\hline $\mathrm{Cr} \mathrm{I}$ & 3.34 & -2.30 & 0.01 & 10 & 0.16 & 2.22 & -3.42 & -0.54 & 4 & 0.17 & 2.77 & -2.87 & -0.34 & 11 & 0.21 \\
\hline Mn I & $<2.40$ & $<-2.99$ & $<-0.68$ & 1 & $\ldots$ & $<2.20$ & $<-3.19$ & $<-0.31$ & 1 & $\ldots$ & 2.20 & -3.19 & -0.66 & 1 & 0.20 \\
\hline $\mathrm{Fe}_{\mathrm{I}}$ & 5.14 & -2.31 & $\ldots$ & 130 & 0.17 & 4.57 & -2.88 & $\ldots$ & 98 & 0.14 & 4.92 & -2.53 & $\ldots$ & 146 & 0.14 \\
\hline Fe II & 5.16 & -2.29 & 0.02 & 18 & 0.18 & 4.57 & -2.88 & 0.00 & 8 & 0.25 & 4.87 & -2.58 & -0.05 & 20 & 0.12 \\
\hline CoI & $<2.69$ & $<-2.23$ & $<0.08$ & 1 & $\ldots$ & $<2.56$ & $<-2.36$ & $<0.52$ & 1 & $\ldots$ & $<2.53$ & $<-2.39$ & $<0.14$ & 1 & $\ldots$ \\
\hline $\mathrm{Ni}$ I & 3.36 & -2.87 & -0.56 & 3 & 0.20 & 3.43 & -2.80 & 0.08 & 5 & 0.22 & 3.51 & -2.72 & -0.19 & 9 & 0.12 \\
\hline $\mathrm{Cu}$ I & $<1.78$ & $<-2.43$ & $<-0.12$ & 1 & $\ldots$ & $<1.52$ & $<-2.69$ & $<0.19$ & 1 & $\ldots$ & $<1.44$ & $<-2.77$ & $<-0.24$ & 1 & $\ldots$ \\
\hline $\mathrm{Zn} \mathrm{I}$ & 2.07 & -2.53 & -0.22 & 1 & 0.20 & 1.78 & -2.82 & 0.06 & 1 & 0.20 & 2.17 & -2.43 & 0.10 & 1 & 0.20 \\
\hline Sr II & -0.80 & -3.72 & -1.41 & 1 & 0.30 & -1.60 & -4.52 & -1.64 & 1 & 0.30 & -1.00 & -3.92 & -1.39 & 1 & 0.30 \\
\hline$Y_{\text {II }}$ & $<-1.19$ & $<-3.40$ & $<-1.09$ & 1 & $\ldots$ & $<-1.17$ & $<-3.38$ & $<-0.50$ & 1 & $\ldots$ & $<-1.57$ & $<-3.78$ & $<-1.25$ & 1 & $\ldots$ \\
\hline Zr II & $<-0.18$ & $<-2.77$ & $<-0.46$ & 1 & $\ldots$ & $<0.05$ & $<-2.54$ & $<0.34$ & 1 & $\ldots$ & $<-0.85$ & $<-3.44$ & $<-0.91$ & 1 & $\ldots$ \\
\hline Ba II & -2.47 & -4.64 & -2.33 & 2 & 0.30 & -2.62 & -4.79 & -1.91 & 2 & 0.30 & -1.67 & -3.84 & -1.31 & 3 & 0.30 \\
\hline La II & $<-1.57$ & $<-2.70$ & $<-0.39$ & 1 & $\ldots$ & $<-1.70$ & $<-2.83$ & $<0.05$ & 1 & $\ldots$ & $<-2.08$ & $<-3.21$ & $<-0.68$ & 1 & $\ldots$ \\
\hline Ce II & $<-1.17$ & $<-2.75$ & $<-0.44$ & 1 & $\ldots$ & $<-1.34$ & $<-2.92$ & $<-0.04$ & 1 & & $<-1.69$ & $<-3.27$ & $<-0.74$ & 1 & $\ldots$ \\
\hline Nd II & $<-0.88$ & $<-2.33$ & $<-0.02$ & 1 & $\ldots$ & $<-0.99$ & $<-2.44$ & $<0.44$ & 1 & $\ldots$ & $<-1.32$ & $<-2.77$ & $<-0.24$ & 1 & $\ldots$ \\
\hline Sm II & $<-1.18$ & $<-2.19$ & $<0.12$ & 1 & $\ldots$ & $<-1.35$ & $<-2.36$ & $<0.52$ & 1 & $\ldots$ & $<-1.71$ & $<-2.72$ & $<-0.19$ & 1 & $\ldots$ \\
\hline Eu II & $<-1.80$ & $<-2.32$ & $<-0.01$ & 1 & $\ldots$ & $<-2.40$ & $<-2.92$ & $<-0.04$ & 1 & $\ldots$ & $<-2.40$ & $<-2.92$ & $<-0.39$ & 1 & $x \quad \ldots$ \\
\hline
\end{tabular}

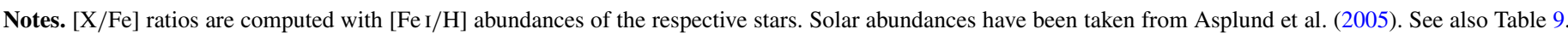
For abundances measured from only one line, we adopt a nominal uncertainty of 0.20 dex.

Table 8

Example Abundance Uncertainties for ComBer-S3

\begin{tabular}{|c|c|c|c|c|c|}
\hline Element & $\begin{array}{c}\text { Random } \\
\text { Uncertainty }\end{array}$ & $\begin{array}{c}\Delta T_{\text {eff }} \\
+200 \mathrm{~K} \\
\end{array}$ & $\begin{array}{c}\Delta \log g \\
+0.4 \mathrm{dex}\end{array}$ & $\begin{array}{c}\Delta v_{\text {micr }} \\
+0.3 \mathrm{~km} \mathrm{~s}^{-1}\end{array}$ & $\begin{array}{c}\text { Total } \\
\text { Uncertainty }^{\mathrm{b}}\end{array}$ \\
\hline $\mathrm{C}(\mathrm{CH})$ & 0.20 & 0.40 & -0.10 & -0.02 & 0.46 \\
\hline $\mathrm{NaI}$ & 0.30 & 0.29 & -0.17 & -0.13 & 0.47 \\
\hline $\mathrm{Mg}_{\mathrm{I}}$ & 0.10 & 0.20 & -0.08 & -0.06 & 0.24 \\
\hline $\mathrm{Ca}$ & 0.15 & 0.14 & -0.07 & -0.06 & 0.22 \\
\hline Sc II & 0.10 & 0.09 & 0.09 & -0.06 & 0.15 \\
\hline Ti I & 0.12 & 0.28 & -0.07 & -0.03 & 0.31 \\
\hline Ti II & 0.16 & 0.06 & 0.09 & -0.08 & 0.21 \\
\hline CrI & 0.21 & 0.28 & -0.09 & -0.06 & 0.37 \\
\hline Mn I & 0.20 & 0.19 & -0.06 & -0.01 & 0.28 \\
\hline $\mathrm{Fe}_{\mathrm{I}}$ & 0.14 & 0.25 & -0.08 & -0.07 & 0.31 \\
\hline Fe II & 0.12 & 0.02 & 0.10 & -0.06 & 0.17 \\
\hline $\mathrm{Ni}$ I & 0.12 & 0.22 & -0.04 & -0.01 & 0.25 \\
\hline $\mathrm{Zn} \mathrm{I}$ & 0.20 & 0.06 & 0.04 & -0.02 & 0.21 \\
\hline Sr II & 0.20 & 0.11 & 0.04 & -0.19 & 0.37 \\
\hline Ba II & 0.15 & 0.14 & 0.10 & -0.06 & 0.35 \\
\hline
\end{tabular}

Notes.

a Standard deviation of individual line abundances (as given in Tables 6 and 7). For elements with just one line we adopt a nominal random uncertainty of 0.20 dex.

b Obtained by adding all uncertainties in quadrature.

first compare the codes with no scatter treatment in place. The difference in abundances $\left(\log \epsilon(\mathrm{X})^{10}\right)$ for individual lines is less than $0.03 \mathrm{dex}$ for the vast majority of lines. We checked that

${ }^{10} \epsilon(\mathrm{X})=\log \left(N_{\mathrm{X}} / N_{\mathrm{H}}\right)+12.0$ the relative abundances $[\mathrm{X} / \mathrm{Fe}]$ are not significantly affected. In Figure 9, we show the abundance differences as a function of wavelength and equivalent width. As can be seen, significant deviations $(\sim 0.1-0.2 \mathrm{dex})$ are only present for the strongest lines

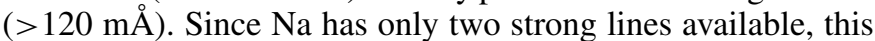
element would be the only one affected systematically. However, since ComBer-S3 has the strongest $\mathrm{Na}$ lines in the sample the effect would likely be less pronounced in the more metal-poor stars. For the comparison with the Cayrel et al. (2004) sample in Figure 10, we apply a constant offset of +0.15 dex to all our $\mathrm{Na}$ abundances since the Cayrel et al. study employed model atmospheres more closely related to the MARCS model we used for this test.

Since Cayrel et al. (2004) use a model atmosphere code that accounts for the scattering, we also investigate what the gross effect of the simplified treatment in MOOG would be. Using BSYN with the proper scatter treatment, find the known dependency of abundance with wavelength (bluer lines are more affected than red lines). However, since we have relatively few lines with wavelengths bluer than $\sim 4500 \AA$ and most elements also (or exclusively) have lines at redder wavelengths, the bias is small. Since we are using relative abundances $[\mathrm{X} / \mathrm{Fe}]$, these abundance ratios are even less affected. We quantify the differences as follows: $\mathrm{Mg}, \mathrm{Ca}$, $\mathrm{Ti}$ II, and $\mathrm{Cr}$ have an average offset over all lines of $\sim 0.00$ dex (i.e., their $[\mathrm{X} / \mathrm{Fe}]$ ratios are not affected), Ti I, Sc, Mn, and Zn have offsets of $\sim+0.03$ dex, Co and Ni have offsets of $\sim+0.05 \mathrm{dex}$, and $\mathrm{Na}$ has +0.20 dex. Since these offsets are small we do not apply them before plotting our abundances in Figure 10. The only exception 

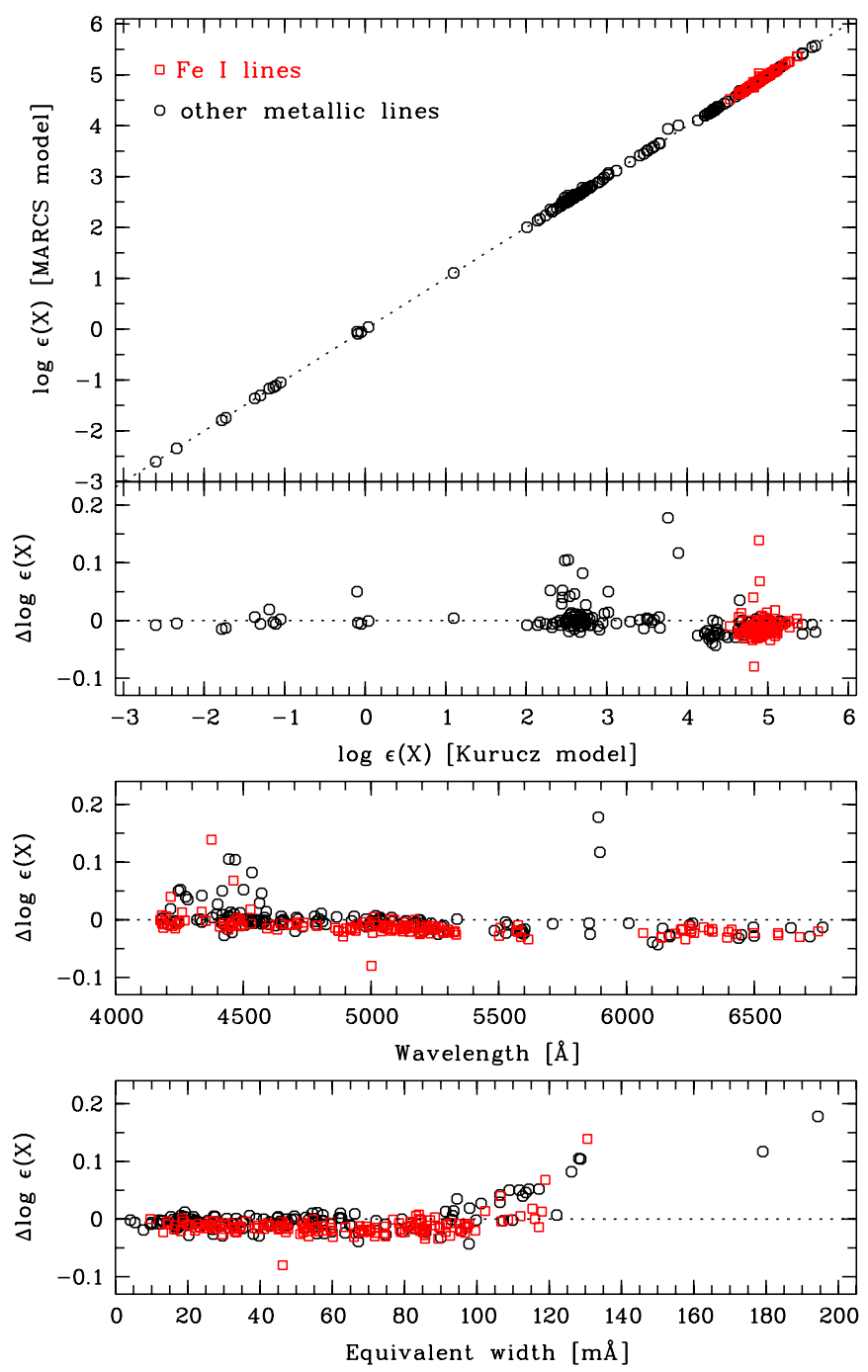

Figure 9. Comparison of abundances of star ComBer-S3 (with stellar parameters $\left.T_{\text {eff }}=4600 \mathrm{~K}, \log g=1.0,[\mathrm{Fe} / \mathrm{H}]=-2.5\right)$ obtained with Kurucz and MARCS model atmospheres. The residuals are also shown as a function of wavelength and equivalent width (bottom two panels). Red squares indicate Fe I lines. Except for the strongest lines there are no significant differences between the two model atmospheres.

(A color version of this figure is available in the online journal.)

is $\mathrm{Na}$ for which we apply both this and the offset determined above (for a total of $+0.35 \mathrm{dex}$ ).

Finally, we note that while the relative abundances are only minimally affected (except for $\mathrm{Na}$ ), this analysis suggests that the lack of scattering treatment in MOOG leads to an overestimate of the $\mathrm{Fe}$ abundances (both $[\mathrm{Fe} \mathrm{I} / \mathrm{H}]$ and $[\mathrm{Fe} \mathrm{II} / \mathrm{H}]$ ) of our sample by 0.1 dex. This fact supports our finding of two stars with $[\mathrm{Fe} / \mathrm{H}] \lesssim-3.0$ metallicities since we are adopting slightly more conservative values.

Informed by all these tests, we conclude that the choice of model atmosphere is a negligible source of error, but that the different treatment of scattering can lead to small systematic offsets. For very strong lines, and when the majority of lines for an element are located below $\sim 4300 \AA$, these differences are more pronounced. Because few of our abundances rely on such blue lines (and those measurements already have appropriately large uncertainties because of the $\mathrm{S} / \mathrm{N}$ ), this effect is not a concern for the present study.

\subsection{Comparison with HD 122563}

One of the main objectives of this study is to compare stellar abundances from dwarf galaxies with those of MW halo stars. To verify that our measurements are on the standard abundance scale, we observed the archetypal metal-poor halo giant HD 122563 and analyzed its spectrum in the same way as for the other stars. The metallicity of this $\operatorname{star}([\mathrm{Fe} / \mathrm{H}] \sim-2.8)$ is roughly in the middle of the range covered by our program stars, and its temperature is also very similar. Its stellar parameters are listed in Table 5. In Table 9, we list both our [X/Fe] abundances and those of the recent Aoki et al. (2007b) study (supplemented with neutron-capture abundances from Honda et al. 2006). Considering that we derived a temperature that differs from that of Aoki et al. (2007b) by $100 \mathrm{~K}$ (Aoki et al. find $T_{\text {eff }}=4600$, $\log g=1.1,[\mathrm{Fe} / \mathrm{H}]=-2.6$, and $v_{\text {micr }}=2.2$; Honda et al. find $T_{\text {eff }}=4570, \log g=1.1,[\mathrm{Fe} / \mathrm{H}]=-2.77$, and $v_{\text {micr }}=2.2$ ), the abundance ratios we measure generally agree well. The good agreement for most elements lends confidence that our stellar abundance measurements for the ultra-faint dwarf galaxies are reliable and can be meaningfully compared with halo stars to investigate any potential chemical differences.

\section{ABUNDANCES}

In this section, we discuss the detailed abundance measurements for each star in our sample and compare our results with MW halo stars of similar Fe abundances. The metal-poor stars in the MW halo are the only known population with metallicities as low as what is found in the ultra-faint dwarf galaxies. A useful comparison between the abundance patterns of the two samples requires that the data sets cover as similar a range in $\mathrm{Fe}$ abundance as possible. Among the highest quality data sets of metal-deficient MW halo giants available is the sample of high S/N, high-resolution Very Large Telescope/Ultraviolet and Visual Echelle Spectrograph (VLT/UVES) spectra of 32 stars analyzed by Cayrel et al. (2004; C to Zn) and François et al. (2007; neutron-capture elements). A comparison of individual abundance measurements in the ultra-faint dwarf galaxy stars in our study and the Cayrel et al. and François et al. MW halo stars is presented in Figures 10 and 11.

We begin each subsection below with a brief summary of the various nucleosynthesis processes that produce the element or elements in question. Woosley \& Weaver (1995) give an extensive description of the production pathways for the interested reader. A short summary can also be found in Cayrel et al. (2004).

\subsection{Carbon}

Carbon is produced in the triple- $\alpha$ process during helium burning on the RGB. Through dredge-up processes carbon is transported to the surface where it can be lost to the interstellar medium (ISM) if strong stellar winds are present. Carbon is also expelled in supernova explosions. The levels of $\mathrm{C}$ are driven by the explosion energy or the amount of stellar rotation, and also depend on the mass of the progenitor. Measured C abundances in the most metal-poor stars thus provide important fossil information on the various previous (early) enrichment events and the nature of the first stars. Rotating, massive Population III stars (Meynet et al. 2006) may, for example, have been significant producers of the first enrichments in carbon, nitrogen, and oxygen elements.

We measure the $\mathrm{C}$ abundances of the dwarf galaxy stars from the $G$-band head $(\sim 4313 \AA$ ) and the $\mathrm{CH}$ band at $4323 \AA$. Except 

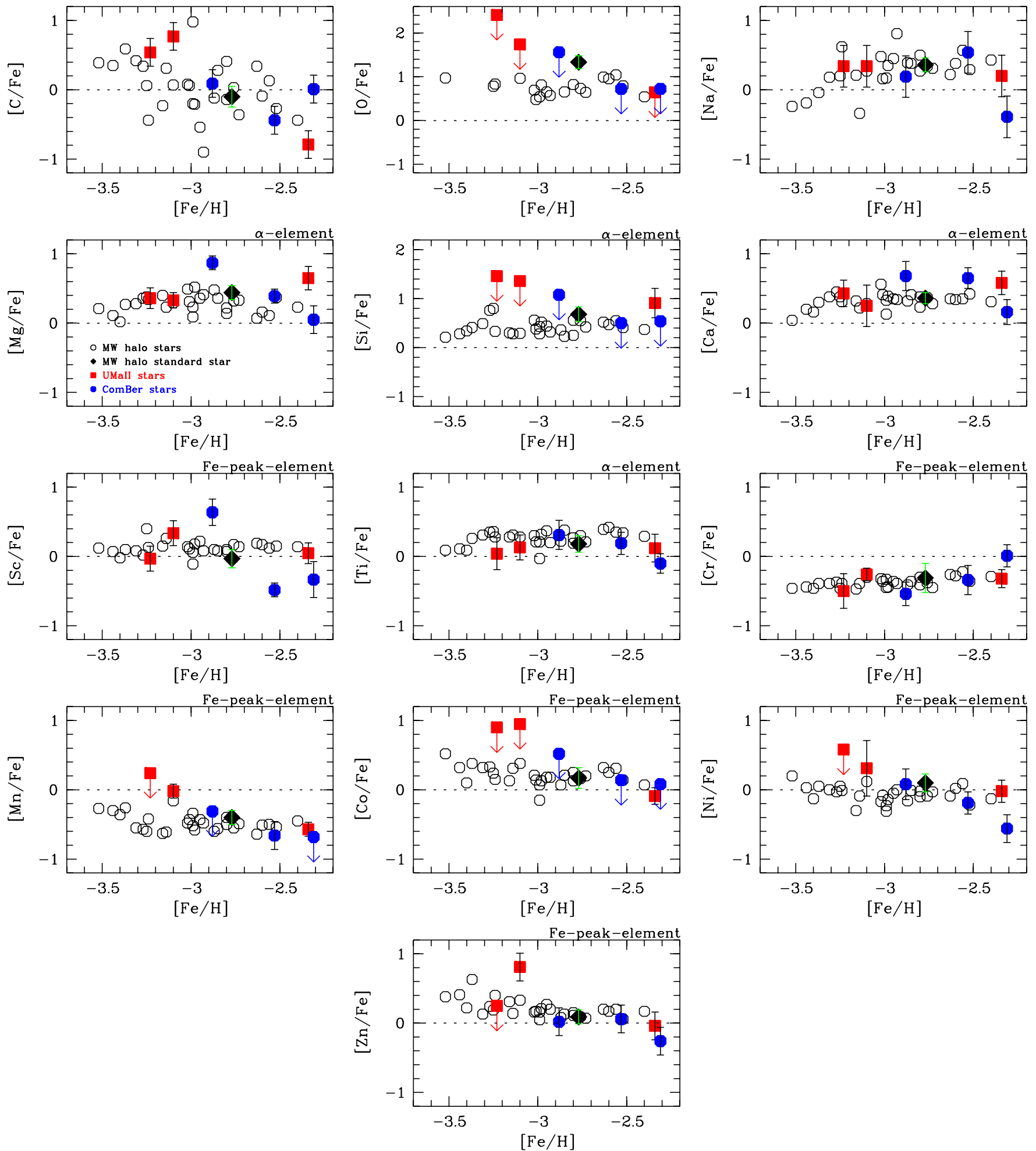

Figure 10. Abundance ratios $([\mathrm{X} / \mathrm{Fe}])$ as a function of metallicity $([\mathrm{Fe} / \mathrm{H}])$ for light and iron-peak elements in comparison with those of Cayrel et al. (2004). The $y$-axes of each panel have the same scale except for O and Si. See Section 3.2 for the discussion. Red squares indicate UMa II stars, blue circles show ComBer stars, open black circles are the Cayrel et al. halo sample, and HD 122563, our MW halo "standard" star, is shown by a black diamond.

(A color version of this figure is available in the online journal.)

for one measurement in the star Dra 119 (Fulbright et al. 2004), no other stellar $\mathrm{C}$ abundances are available in any of the dSphs or other dwarf galaxies. To determine the $\mathrm{C}$ abundance, we compute synthetic spectra with different $\mathrm{C}$ abundances and find the best fit with the observed spectrum. An example of the $\mathrm{CH} \lambda 4323$ synthesis is shown in Figure 12. The values we obtain for the two $\mathrm{CH}$ bands generally agree with each other within $\sim 0.2$ dex, and we adopt the average of the two measurements as our final $\mathrm{C}$ abundance. The dominant source of uncertainty for abundances derived from molecular features is the continuum placement, especially in lower $\mathrm{S} / \mathrm{N}$ spectra. We thus assign a random uncertainty of $\sigma[\mathrm{C} / \mathrm{Fe}]=0.20$. To check 
Table 9

HIRES Abundances of Standard Star HD 122563

\begin{tabular}{|c|c|c|c|c|c|c|c|c|c|}
\hline \multirow[b]{2}{*}{ Species } & \multirow[b]{2}{*}{$\log \epsilon(\mathrm{X})_{\odot}$} & \multicolumn{5}{|c|}{ HD 122563-This Study } & \multicolumn{3}{|c|}{ Aoki et al. (2007b) } \\
\hline & & $\log \epsilon(\mathrm{X})$ & {$[\mathrm{X} / \mathrm{H}]$} & {$[\mathrm{X} / \mathrm{Fe}]$} & $N$ & $\sigma$ & $\log \epsilon(\mathrm{X})$ & {$[\mathrm{X} / \mathrm{H}]$} & {$[\mathrm{X} / \mathrm{Fe}]$} \\
\hline $\mathrm{C}$ & 8.39 & 5.55 & -2.84 & -0.07 & 2 & 0.15 & 5.45 & -2.94 & -0.35 \\
\hline $\mathrm{O}_{\mathrm{I}}$ & 8.66 & 6.40 & -2.26 & 0.51 & 1 & 0.15 & 7.03 & -1.63 & 1.14 \\
\hline $\mathrm{Na} I$ & 6.17 & 3.41 & -2.76 & 0.01 & 2 & 0.10 & 3.39 & -2.78 & -0.19 \\
\hline $\operatorname{Mg}_{\mathrm{I}}$ & 7.53 & 5.31 & -2.22 & 0.55 & 3 & 0.10 & 5.44 & -2.09 & 0.50 \\
\hline $\mathrm{Al} \mathrm{I}$ & 6.37 & $<3.87$ & $<-2.50$ & $<0.27$ & 1 & .. & 3.38 & -2.99 & -0.40 \\
\hline Si I & 7.51 & 5.42 & -2.09 & 0.68 & 1 & 0.15 & 5.34 & -2.17 & 0.41 \\
\hline $\mathrm{CaI}$ & 6.31 & 3.85 & -2.46 & 0.31 & 20 & 0.08 & 3.93 & -2.38 & 0.21 \\
\hline Sc II & 3.05 & 0.16 & -2.89 & -0.12 & 5 & 0.13 & 0.57 & -1.79 & 0.80 \\
\hline Ti I & 4.90 & 2.27 & -2.63 & 0.14 & 21 & 0.07 & 2.42 & -2.48 & 0.11 \\
\hline Ti II & 4.90 & 2.40 & -2.50 & 0.27 & 31 & 0.12 & 2.58 & -2.32 & 0.27 \\
\hline $\mathrm{V}_{\mathrm{I}}$ & 4.00 & 1.13 & -2.87 & -0.10 & 1 & 0.15 & $\ldots$ & $\ldots$ & $\ldots$ \\
\hline Cr I & 5.64 & 2.56 & -3.08 & -0.31 & 12 & 0.21 & 2.57 & -3.07 & -0.48 \\
\hline Mn I & 5.39 & 2.26 & -3.13 & -0.36 & 3 & 0.08 & 2.23 & -3.16 & -0.57 \\
\hline $\mathrm{Fe} I$ & 7.45 & 4.68 & -2.77 & $\ldots$ & 141 & 0.12 & 4.86 & -2.59 & $\ldots$ \\
\hline Fe II & 7.45 & 4.67 & -2.78 & -0.01 & 20 & 0.10 & 4.87 & -2.58 & 0.01 \\
\hline CoI & 4.92 & 2.32 & -2.60 & 0.17 & 1 & 0.15 & 2.48 & -2.44 & 0.15 \\
\hline $\mathrm{Ni} I$ & 6.23 & 3.56 & -2.67 & 0.10 & 11 & 0.13 & 3.66 & -2.57 & 0.02 \\
\hline $\mathrm{Cu} \mathrm{I}$ & 4.21 & $<0.20$ & $<-4.01$ & $<-1.24$ & 1 & $\ldots$ & $\ldots$ & $\ldots$ & $\ldots$ \\
\hline $\mathrm{ZnI}$ & 4.60 & 1.96 & -2.64 & 0.13 & 2 & 0.10 & 2.08 & -2.52 & 0.07 \\
\hline Sr II & 2.92 & -0.40 & -3.32 & -0.55 & 1 & 0.15 & 0.08 & -2.84 & -0.25 \\
\hline Y II & 2.21 & -1.07 & -3.28 & -0.51 & 2 & 0.07 & $-0.93^{\mathrm{a}}$ & -3.14 & -0.37 \\
\hline Zr II & 2.59 & -0.28 & -2.87 & -0.11 & 2 & 0.10 & $-0.28^{\mathrm{a}}$ & -2.87 & -0.10 \\
\hline Ba II & 2.17 & -1.77 & -3.94 & -1.17 & 3 & 0.10 & -1.69 & -3.86 & -1.27 \\
\hline Ce II & 1.58 & -2.00 & -3.58 & -0.81 & 1 & 0.15 & $-1.83^{\mathrm{a}}$ & -3.41 & -0.64 \\
\hline $\mathrm{Nd} \mathrm{II}$ & 1.45 & $<-2.22$ & $<-3.67$ & $<-0.90$ & 1 & $\ldots$ & $-2.01^{\mathrm{a}}$ & -3.46 & -0.69 \\
\hline Sm II & 1.01 & -2.37 & -3.38 & -0.61 & 1 & 0.15 & $-2.16^{\mathrm{a}}$ & -3.17 & -0.40 \\
\hline Eu II & 0.52 & -2.46 & -2.98 & -0.21 & 1 & 0.15 & $-2.77^{\mathrm{a}}$ & -3.29 & -0.52 \\
\hline
\end{tabular}

Notes. $[\mathrm{X} / \mathrm{Fe}]$ ratios are computed using the $[\mathrm{Fe} \mathrm{I} / \mathrm{H}]$ abundance. Solar abundances have been taken from Asplund et al. (2005).

a Neutron-capture abundances are taken from Honda et al. (2006).

the validity of our abundance scale we also determined the $\mathrm{C}$ abundance of HD 122563 (lower panel of Figure 12). We derive an abundance of $[\mathrm{C} / \mathrm{Fe}]=-0.1 \pm 0.1$ for this star. Bearing in mind that we adopted somewhat different stellar parameters, and that abundances from molecular features are quite temperature sensitive, this result is in very good agreement with the value of $[\mathrm{C} / \mathrm{Fe}]=-0.35 \pm 0.2$ determined by Aoki et al. (2007b).

We note that it is not possible to determine a ${ }^{12} \mathrm{C} /{ }^{13} \mathrm{C}$ ratio in our stars. The bottleneck for such measurements is the availability of ${ }^{13} \mathrm{C}$-lines. They are located at 4217.6 and $4225.2 \AA$ where the $\mathrm{S} / \mathrm{N}$ is not sufficient to determine meaningful values or limits of these usually very weak features (even for the stars that have the highest $\mathrm{C}$ abundances in our sample).

As seen in Figure 7, all our targets are on the upper RGB, since less luminous stars in these galaxies are too faint to be observed at high resolution. However, their somewhat evolved nature may lead to concerns regarding the potential for altered surface abundances. Such modifications (intrinsically through dredge-up of nucleosynthesis products or extrinsically through binary mass transfer), if not sufficiently quantified, could lead to incorrect interpretations of the chemical nature of the gas from which these stars formed. We note that in halo field stars, it can be assumed that the abundances of elements other than $\mathrm{C}$ and $\mathrm{N}$ are not affected by early signs of mixing (e.g., Spite et al. 2006). In the absence of a measured ${ }^{12} \mathrm{C} /{ }^{13} \mathrm{C}$ ratio (which is often used in infer details on the degree of atmospheric mixing and stellar evolutionary effects) in our stars, we turn to the luminosities of our targets to gain information on these effects. In Figure 13 (top panel), we show the $\mathrm{C}$ abundances of our stars compared with mixed $^{11}$ (solid circles) and unmixed (open circles) metal-poor giants (Cayrel et al. 2004, also analyzed by Spite et al. 2006). Their mixed giants are typical halo stars that have ascended the giant branch far enough that early signs of mixing can be observed. As a star moves up the giant branch, carbon decreases as a consequence of the $\mathrm{CN}$ cycling that converts $\mathrm{C}$ to $\mathrm{N}$. This processing results in a drop of the $\mathrm{C}$ abundances with increasing stellar luminosity (Gratton et al. 2000). Depletion values can be as high as $\sim-0.7$ dex for cool upper RGB stars such as our targets. The middle panel of Figure 13 shows the dwarf galaxy $\mathrm{C}$ abundances compared with various halo stars from the literature to allow the reader to appreciate where the stars in our dwarf galaxies are located with respect to the present range of halo data. Our $\mathrm{C}$ abundances agree well with those of the mixed halo giants, except for the two most metal-poor stars in our sample. Those stars have much higher $\mathrm{C}$ abundances, similar to what is found for unmixed stars. However, all our stars have lower effective temperatures (i.e., the stars are further evolved) than those of Cayrel et al. (2004), which suggests that all of them must have undergone some degree of mixing, even the two most metal-poor stars. This "discrepancy" thus indicates that they appear to be C-enhanced beyond what is expected from canonical stellar evolution (i.e., the level of the mixed stars). This would mean that the stars must have been born from material that was overabundant in carbon. We note that the Spite et al. stars are

\footnotetext{
11 The separation of mixed and unmixed giants is based on a cut in $\mathrm{N}$ abundances at $[\mathrm{N} / \mathrm{Fe}]=0.58$, with the mixed stars having higher $\mathrm{N}$ abundances Spite et al. (2006).
} 

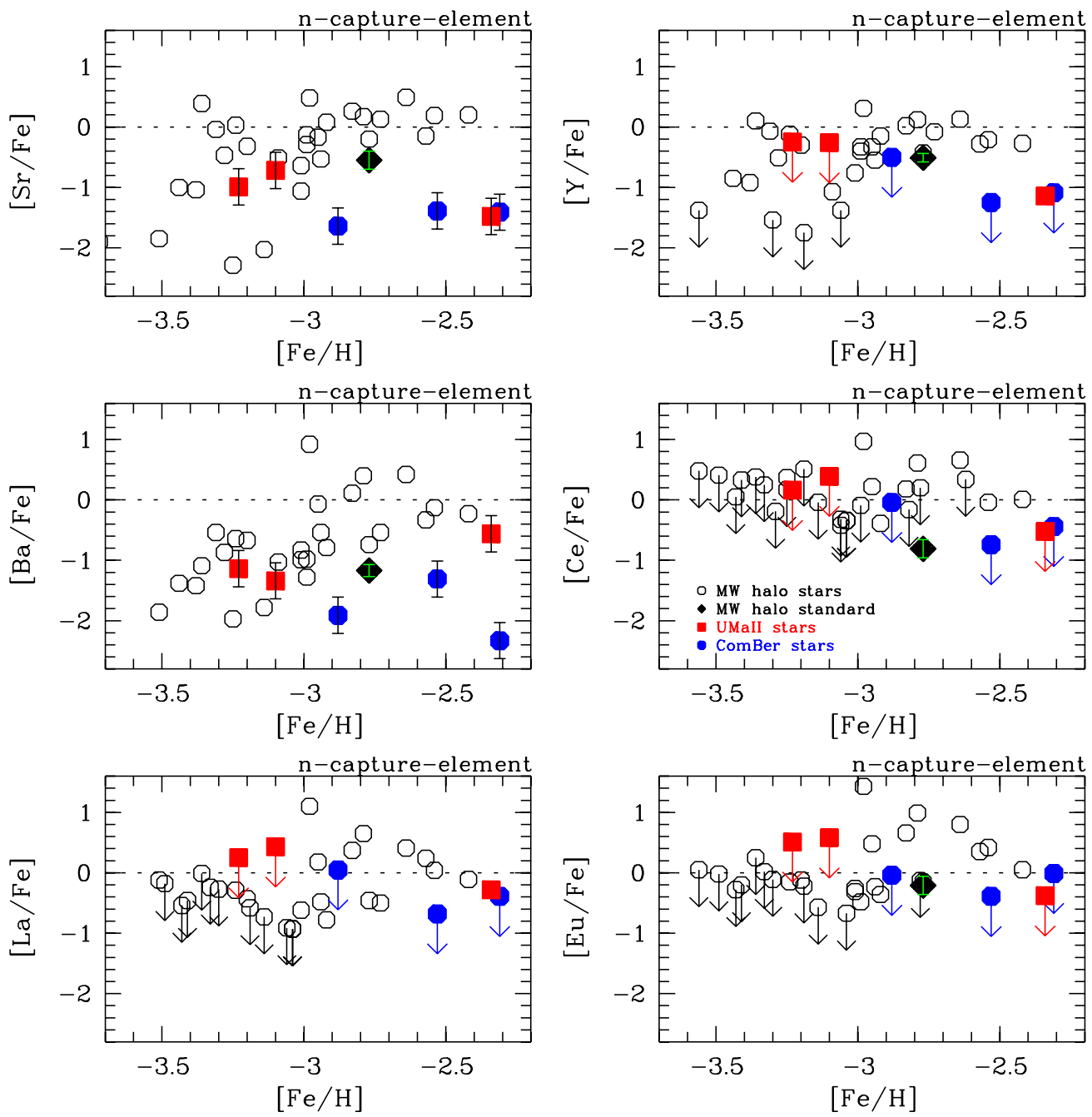

Figure 11. Abundance ratios $([\mathrm{X} / \mathrm{Fe}])$ as a function of metallicity $([\mathrm{Fe} / \mathrm{H}])$ for neutron-capture elements in comparison with those of Cayrel et al. (2004). See Section 3.3 for the discussion. Red squares indicate UMa II stars, blue circles show ComBer stars, open black circles are the Cayrel et al. halo sample, and HD 122563, our MW halo "standard" star, is shown by a black diamond. Note that in the bottom plot, a Cayrel et al. star has an upper limit at the position of the Eu abundance of HD 122563.

(A color version of this figure is available in the online journal.)

not C-enriched, i.e., they formed from gas that was not enriched in carbon beyond the general level provided by the chemical evolution at that time. The common definition of C-rich metalpoor stars is $[\mathrm{C} / \mathrm{Fe}]>1.0$ (e.g., Beers \& Christlieb 2005), assuming that the currently observed $\mathrm{C}$ abundance reflects the abundance of the birth material. Our stars do not quite reach this level, but as we discuss below, for upper RGB this "onesize-fits-all" definition may not be appropriate because mixing processes change the surface $\mathrm{C}$ abundances we observe today.

In the bottom panel of Figure 13, we plot the $[\mathrm{C} / \mathrm{Fe}]$ abundances as a function of luminosity. Taking the luminosity dependent C-decrease into account, Aoki et al. (2007a) suggested redefining the classification of $\mathrm{C}$-rich stars to

$$
[\mathrm{C} / \mathrm{Fe}] \geqslant+0.7 \text { for stars with } \log \left(L / L_{\odot}\right) \leqslant 2.3
$$

and

$$
[\mathrm{C} / \mathrm{Fe}] \geqslant+3.0-\log \left(L / L_{\odot}\right) \text { when } \log \left(L / L_{\odot}\right)>2.3
$$

This boundary is indicated in Figure 13 (dashed line). As can be seen, all three members of ComBer are not enriched in C; in fact, they all must have had very similar $\mathrm{C}$ abundances at birth that by now have decreased to the observed level because of their large luminosities. The two most metal-poor stars (both in UMa II), on the other hand, can be classified as C-rich. They contain larger $\mathrm{C}$ abundances than what is expected for stars at larger luminosities (i.e., low effective temperatures) in which some mixing has taken place. The third UMa II object, however, is rather like the ComBer stars, i.e., it shows a decrease in $\mathrm{C}$ consistent with its luminosity and must have been born from material that was not especially enriched in carbon. This strongly suggests a large spread of $\mathrm{C}$ abundances in UMa II, which could point to either different production sites and timescales (the C-normal star is more metal-rich than the C-rich stars) or varying degrees of mixing in this galaxy. We see no obvious correlation between $\mathrm{Fe}$ abundance and the galactocentric distance of the stars. This indicates that an ad hoc assumption of incomplete mixing in the ISM may not explain the very large $\mathrm{Fe}$ and $\mathrm{C}$ spread. However, with the small existing sample of stars and no knowledge about the actual physical boundaries and dynamical history of these systems we can only speculate whether the present location of our targets would reveal anything at all about mixing processes. More stars in both of these systems are clearly needed to provide more insight into this issue. Nevertheless, the fact that two of our six 

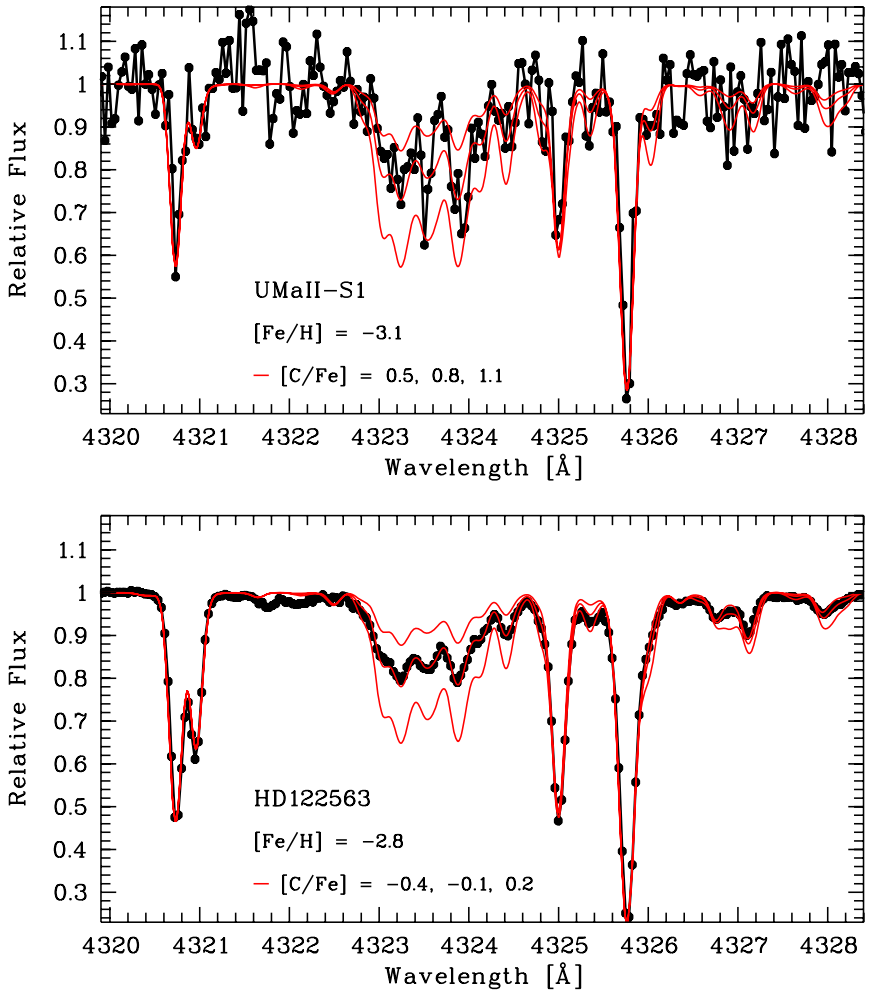

Figure 12. Spectral region around the $\mathrm{CH}$ feature at $4323 \AA$ for UMa II-S1 (upper panel) and HD 122563 (lower panel). The observed spectrum is shown (thick line plus filled symbols). Synthetic spectra with three different $\mathrm{C}$ abundances are shown in red. In addition to the Draco star Dra119 (Fulbright et al. 2004), these are the first carbon abundances measured in a dwarf galaxy.

(A color version of this figure is available in the online journal.)

stars are C-rich is very interesting in itself. As has been known for quite a while, a large fraction of metal-poor halo stars are enriched in $\mathrm{C}$, with numbers ranging from $\sim 15 \%$ (Frebel et al. 2006b; Cohen et al. 2005) to 25\% (Marsteller et al. 2005). Below $[\mathrm{Fe} / \mathrm{H}]<-3.0$, these numbers are found to increase in all samples, although those results are generally plagued by small-number statistics. Hence, finding two C-rich stars in a dwarf galaxy, which are also the two most metal-poor stars in our sample and among the most metal-poor ones in the entire sample of Kirby et al. (2008), is suggestive of a high fraction of C-rich stars in dwarf galaxies as well. Although the statistical significance of this result is low, it may indicate that $\mathrm{C}$ generally played an important role in the formation and evolutionary process at early times irrespective of the host galaxy system.

\subsection{Elements with $Z \leqslant 30$}

Light elements are produced during stellar evolution or directly in supernovae and then expelled during the explosions (e.g., Woosley \& Weaver 1995; Nomoto et al. 1997).

\subsubsection{Sodium}

Sodium is produced during carbon burning and through the $\mathrm{Ne}-\mathrm{Na}$ cycle during $\mathrm{H}$ burning (Woosley \& Weaver 1995). It has thus been suggested that $\mathrm{Na}$ correlates with $\mathrm{Ni}$ since the $\mathrm{Ni}$ production depends on the neutron excess provided by ${ }^{23} \mathrm{Na}$ during the supernova explosion that drives the ${ }^{58} \mathrm{Ni}$ abundances (see also Venn et al. 2004). This hypothesis would explain why both elements are observed to have similar abundances in stars.

The Na I D resonance lines at $\sim 5890 \AA$ are used to determine the $\mathrm{Na}$ abundances. The resonance lines are very sensitive
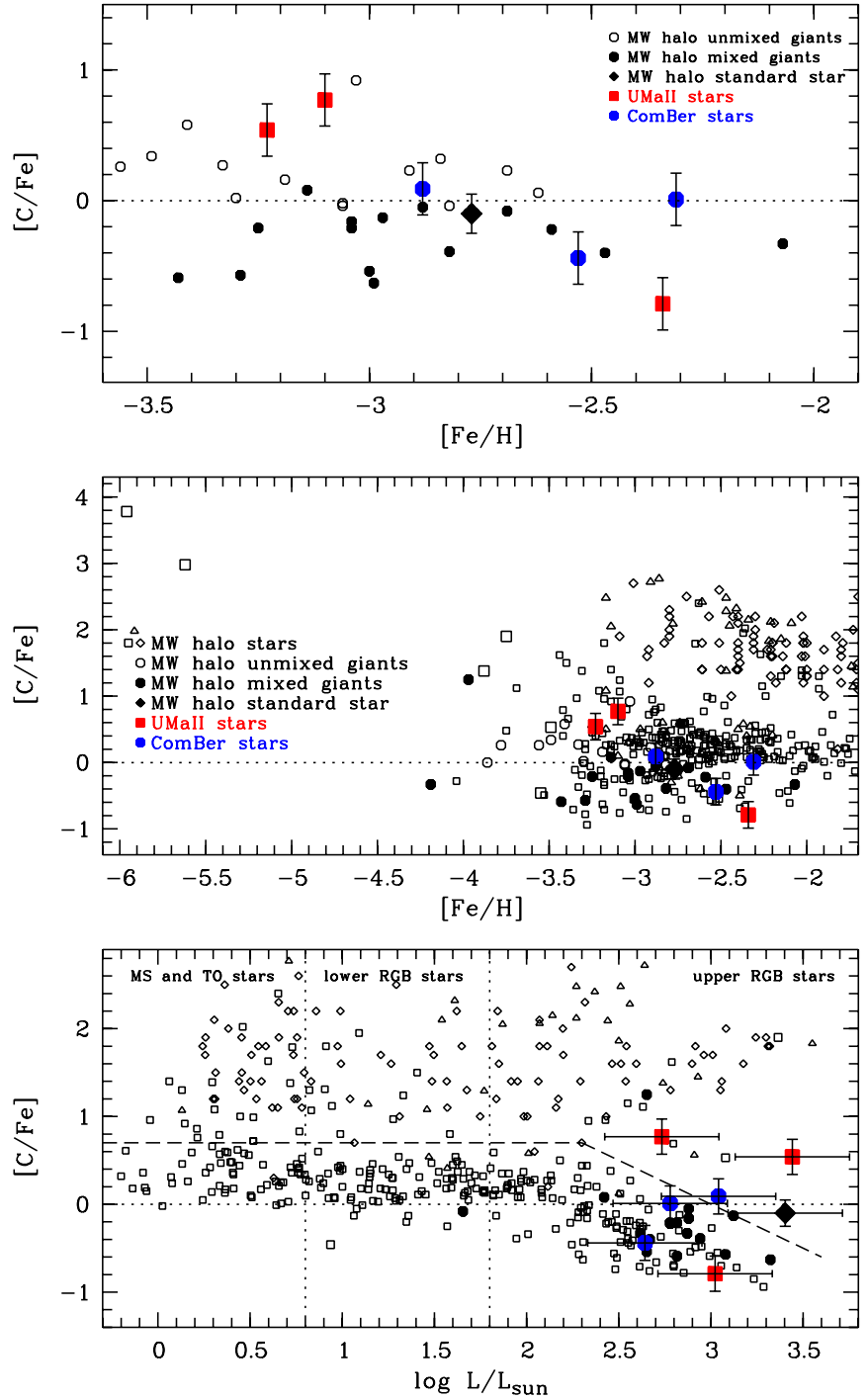

Figure 13. $[\mathrm{C} / \mathrm{Fe}]$ abundance ratios as a function of $[\mathrm{Fe} / \mathrm{H}]$ (top and middle panels) and luminosity (bottom panel). In all three panels, blue circles indicate ComBer stars, red squares show UMa II objects, and the black diamond is our MW halo standard HD 122563. The top panel compares our C abundances to MW halo giants (Spite et al. 2006; Aoki et al. 2007a) to assess the level of atmospheric mixing which can affect the carbon abundances. Middle panel: The same comparison is shown with an expanded comparison sample (squares: Aoki et al. 2002; Cohen et al. 2005; Barklem et al. 2005; Collet et al. 2006; Frebel et al. 2007b, 2008; Lai et al. 2008; triangles: Lucatello et al. 2006; Aoki et al. 2005). Bottom panel: $[\mathrm{C} / \mathrm{Fe}]$ ratios as a function of luminosity. Indicated are different luminosity bins for each evolutionary stage. The definition of $\mathrm{C}$ richness from Aoki et al. (2007a) is also shown (dashed line). All three members of ComBer and the most metal-rich UMa II star (UMa II-S3) are classified as carbon-normal, while the two more metal-poor members of UMa II are carbonrich.

(A color version of this figure is available in the online journal.)

to non-LTE effects. We note that, in principle, all $[\mathrm{Na} / \mathrm{Fe}]$ abundances shown in Figure 10 should be decreased by several tenths of dex (e.g., Baumueller et al. 1998) to account for non-LTE effects. For ease of discussion, however, we simply compare our LTE $\mathrm{Na}$ abundances with the uncorrected (i.e., LTE) abundances of Cayrel et al. (2004). The (LTE) agreement is generally quite good, although our highest metallicity star deviates from the bulk of the halo and our other dwarf galaxy abundances by almost 1 dex. Such low Na abundance is very unusual, and no other stars are known with similarly low $\mathrm{Na}$ values. 


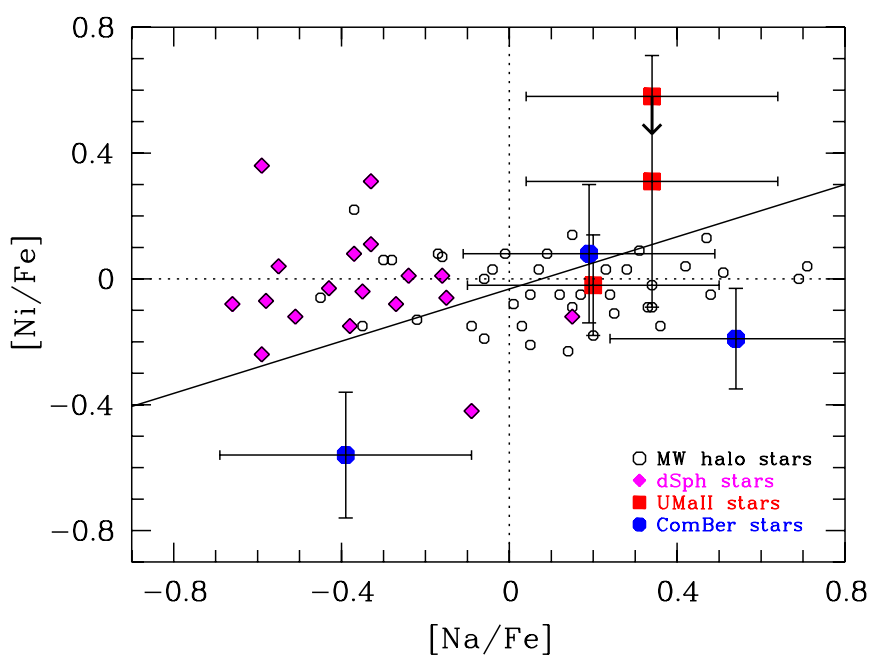

Figure 14. $[\mathrm{Ni} / \mathrm{Fe}]$ ratios for the program stars as function of $[\mathrm{Na} / \mathrm{Fe}]$ in comparison with other objects from the literature (black circles: Cayrel et al. 2004; Lai et al. 2008; pink diamonds: Venn et al. 2004). Blue circles indicate ComBer stars, whereas red squares indicate UMa II stars. The relation found by Nissen \& Schuster (1997) is also shown (solid line). The two UMa II stars that deviate most from the Nissen \& Schuster relation are also the only two carbon-rich stars in the sample.

(A color version of this figure is available in the online journal.)

Nissen \& Schuster (1997) analyzed a disk star sample with somewhat deficient $[\mathrm{Na} / \mathrm{Fe}]$ and $[\mathrm{Ni} / \mathrm{Fe}]$ abundances. Figure 14 illustrates the correlations they found and shows that ComBer-S1 extends this relationship to lower $\mathrm{Na}$ and $\mathrm{Ni}$ values by $\sim 0.5$ dex. Our other stars do not deviate significantly from the suggested correlation. There is a large scatter among the halo stars of Cayrel et al. (2004) (black symbols in the figure), and it is difficult to evaluate whether they generally follow the Nissen \& Schuster (1997) trend. It should also be said that the Cayrel et al. stars are halo stars and have much lower Fe abundances than the stars originally considered for this relationship. Nevertheless, they seem to follow the trend better than the higher-metallicity dSph stars collected by Venn et al. (2004), which generally have higher Ni abundances than predicted by the correlation. This may indicate different nucleosynthetic origins for the stars in the brighter $\mathrm{dSphs}$ and is discussed further below.

\subsection{2. $\alpha$-Elements}

The $\alpha$-elements $(\mathrm{Mg}, \mathrm{Ca}, \mathrm{Si}, \mathrm{Ti})$ are built from multiples of He nuclei since they are produced through $\alpha$-captures during various burning stages of stellar evolution (carbon burning, neon burning, complete and incomplete Si burning) and then dispersed during the explosions of core-collapse supernovae. Although $\mathrm{Ti}(Z=22)$ is not a true $\alpha$-element, in metal-poor stars the dominant isotope is ${ }^{48} \mathrm{Ti}$, which behaves like an $\alpha$ element.

Several Mg I lines across the spectrum were employed to derive the $\mathrm{Mg}$ abundance. In some cases, the $\mathrm{Mg}_{\mathrm{I}} \mathrm{b}$ triplet lines at $\sim 5170 \AA$ were very strong and beyond the linear part of the curve-of-growth. Nevertheless, the abundances of those lines generally agreed with those of the other $\mathrm{Mg}$ lines. Four of our six stars have $\mathrm{Mg}$ abundances of $[\mathrm{Mg} / \mathrm{Fe}] \sim 0.4$, in good agreement (see Figure 10) with the general trend in MW halo stars (e.g., Cayrel et al. 2004; Barklem et al. 2005; Lai et al. 2008). Two stars, however, are overabundant in this element: ComBer-S2 has $[\mathrm{Mg} / \mathrm{Fe}] \sim 1.0$, and UMa II-S3 has $[\mathrm{Mg} / \mathrm{Fe}] \sim 0.7$. Figure 15 shows the spectral region around the a Mg I line at $4703 \AA$ of ComBer-S2 in comparison with ComBer-S3, one of the stars with a lower, MW halo-like $\mathrm{Mg}$ abundance. The stars have the same effective temperature and similar surface gravities, but different $\mathrm{Fe}$ abundances. This leads to the different line strengths for all metals, which can be seen in the figure. The Mg lines, however, have roughly the same strength, illustrating the Mg-rich nature of ComBer-S2. There are a few cases known where metal-poor MW halo stars have large $\mathrm{Mg}$ overabundances of up to $[\mathrm{Mg} / \mathrm{Fe}] \sim 2.0$ (e.g., Aoki et al. 2002; Frebel et al. 2005). Some of these stars are very $\mathrm{C}$-rich as well, but that is not the case for ComBer-S2. Si may also be enhanced in such stars, but unfortunately our spectra do not cover the strong Si line at $3905 \AA$ A. Hence, we are only able to derive upper limits from the much weaker $5684 \AA$ A line. The limit for the most Mg-rich star (ComBer-S2) rules out a Si abundance of $[\mathrm{Si} / \mathrm{Fe}] \gtrsim 1.0$.

The $\mathrm{Ca}$ abundances are shown in Figure 10, derived from several lines of $\mathrm{Ca}$ I. Similar to $\mathrm{Mg}$, the $\mathrm{Ca}$ abundances agree very well with the MW halo trend of $[\mathrm{Ca} / \mathrm{Fe}] \sim 0.4$. The Ti abundances are based on numerous lines across the spectrum. Generally, Ti I values agree within 0.2 dex to those of Ti II, which in turn are in good agreement with the Cayrel et al. (2004) halo pattern (Figure 10). Figure 16 shows a more detailed comparison of our $\alpha$-element results with those of a large set of literature halo stars. While there are some outliers among our more metal-poor stars that deviate from the halo data by having slightly higher abundances, the general trend of the more luminous dSphs to have $[\alpha / \mathrm{Fe}]$ ratios below the halo data is not followed by UMa II and ComBer.

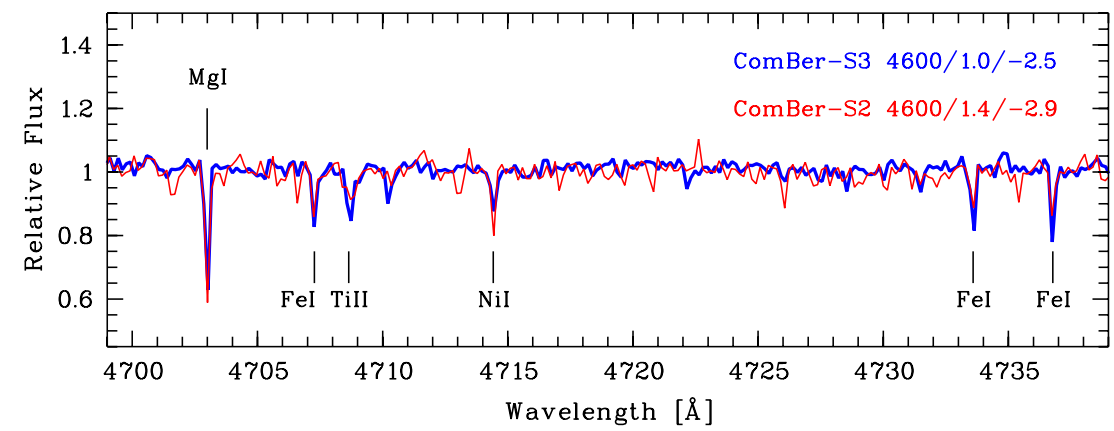

Figure 15. Spectral region around $4700 \AA$ in ComBer-S2 in comparison to ComBer-S3. The stars have similar stellar parameters but different Fe abundances, which is reflected in the different Fe line strengths, except for the $\mathrm{Mg}$ line at $4703 \AA$ which is stronger in ComBer-S2. Several species are labeled, and the atmospheric parameters of both stars are given in the legend.

(A color version of this figure is available in the online journal.) 

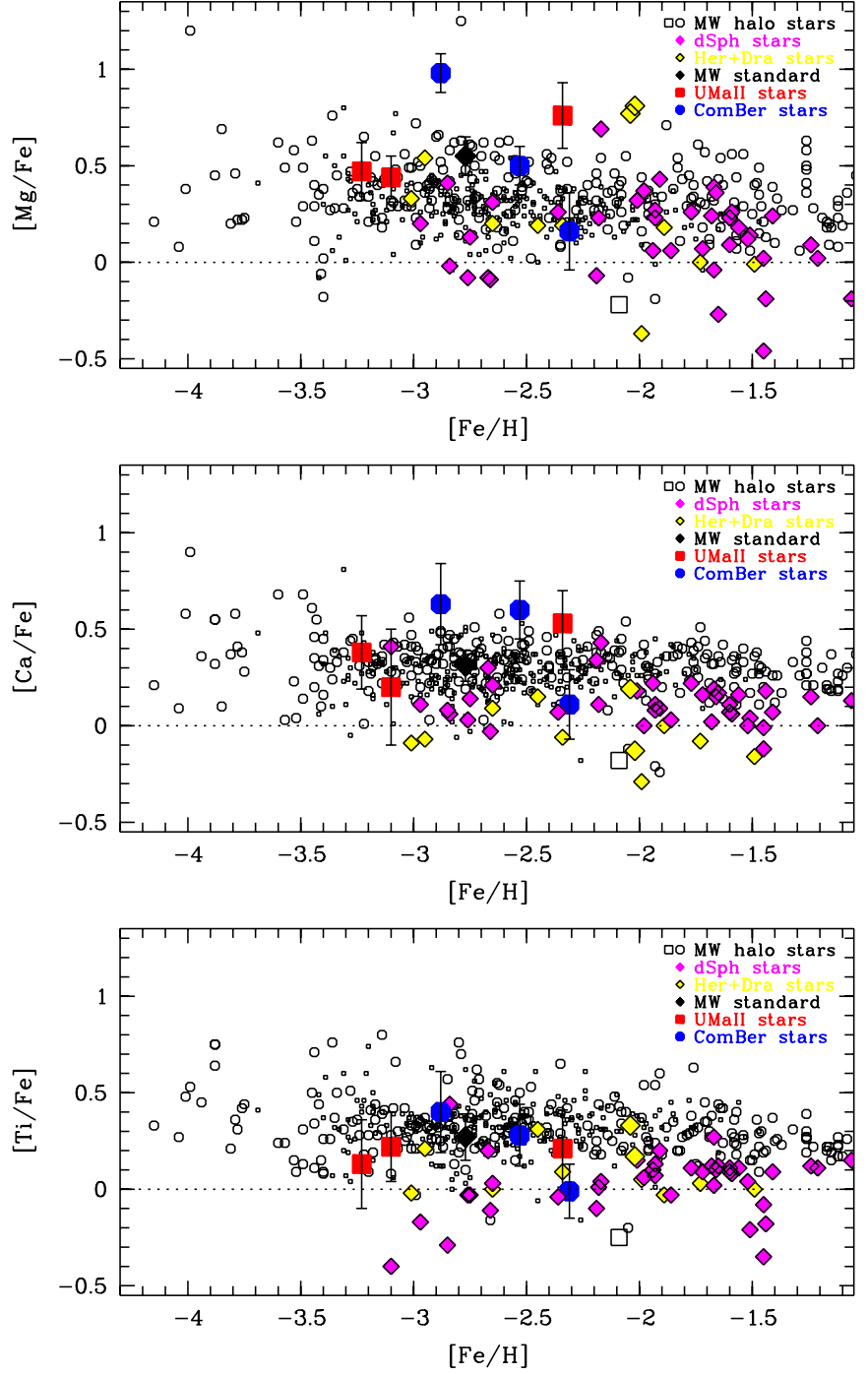

Figure 16. $[\mathrm{Mg} / \mathrm{Fe}]$ (top panel), $[\mathrm{Ca} / \mathrm{Fe}]$ (middle panel), and $[\mathrm{Ti} / \mathrm{Fe}]$ (bottom panel) abundance ratios as a function of $[\mathrm{Fe} / \mathrm{H}]$. Generally, the ultra-faint dwarf galaxy abundances (red squares: UMa II stars; blue circles: ComBer stars) agree with the metal-poor halo abundances (black squares and circles), in contrast to those of the more luminous dSphs (pink diamonds; Shetrone et al. 2001, 2003; Sadakane et al. 2004; Aoki et al. 2009). We also find two stars that are Mg-rich, similar to a few known halo stars. Halo data are taken from Lai et al. (2008), François et al. (2007), Barklem et al. (2005), and Venn et al. (2004). The metalpoor halo standard star HD 122563 is marked with a black diamond. The big yellow diamonds at $[\mathrm{Fe} / \mathrm{H}] \sim-2.0$ are two stars in the ultra-faint dwarf galaxy Hercules (Koch et al. 2008b). The small yellow diamonds refer to the Draco data by Cohen \& Huang (2009) and Draco D119 at $[\mathrm{Fe} / \mathrm{H}] \sim-2.0$ (Fulbright et al. 2004), which show somewhat similar $\mathrm{Mg}$ and Ti abundances to our targets. The open black square indicates the $\alpha$-poor, neutron-capture-poor star BD 80 245 (Ivans et al. 2003).

(A color version of this figure is available in the online journal.)

\subsubsection{Iron-peak Elements}

In the early universe, the iron-peak elements ( $\mathrm{Sc}$ to $\mathrm{Zn}$; $23 \leqslant Z \leqslant 30)$ are exclusively synthesized during Type II supernova (SN II) explosions by explosive oxygen and neon burning, and complete and incomplete explosive Si burning. Only at later times, once the stars less massive than those exploding as $\mathrm{SNe}$ II reach the end of their life time, do SNe Ia became the dominant contributor to the total iron inventory. The onset of SNe Ia in the chemical evolution of the MW halo is clearly observed in the $[\alpha / \mathrm{Fe}]$ versus $[\mathrm{Fe} / \mathrm{H}]$ plane by means of a down-turn of the $[\alpha / \mathrm{Fe}] \sim 0.4$ plateau at metallicities above $[\mathrm{Fe} / \mathrm{H}] \sim-1.5$ (e.g., McWilliam et al. 1995; Ryan et al. 1996).
We measured the Fe-peak elements $\mathrm{Sc}, \mathrm{Cr}, \mathrm{Mn}, \mathrm{Ni}, \mathrm{Fe}$, and $\mathrm{Zn}$ in our dwarf galaxy stars. Overall, there is good agreement between our abundances and those in the halo. Sc and $\mathrm{Mn}$ abundances were determined from several lines. Hyper-fine structure was taken into account (using Kurucz line lists) and the abundances of the lines were derived from spectral synthesis. The Sc abundances in UMa II agree with those of the halo stars, but there is significant scatter found in ComBer (up to $\sim 1$ dex). $\mathrm{Mn}, \mathrm{Ni}$, and $\mathrm{Zn}$ abundances were determined, respectively, in 3, 3 , and 5 of the 6 program stars, and upper limits were derived for the remaining stars. Our measured $\mathrm{Mn}, \mathrm{Cr}, \mathrm{Ni}$, and $\mathrm{Zn}$ abundances generally follow the halo trend. The only exception is the highest metallicity star (ComBer-S1), which has a rather low Mn upper limit and a low, subsolar Ni abundance that can be explained by the low $\mathrm{Na}$ abundance. The most metalpoor star, UMa II-S2, has an unusually high Zn abundance that could indicate a very high explosion energy for the supernovae responsible for its abundance pattern (Umeda \& Nomoto 2002).

\subsubsection{Upper Limits}

Upper limits were determined for $\mathrm{O}, \mathrm{Al}, \mathrm{Si}, \mathrm{V}, \mathrm{Co}$, and $\mathrm{Cu}$. The limits are generally tighter at higher Fe values where the searched-for lines are expected to be stronger. For Co, the limits indicate no enhancement with respect to the halo material among our higher metallicity stars, and perhaps a small deficit. We note for completeness that all our stars are too evolved to show any detectable Li in their spectra. Because of the increased thickness of the convection zone as the stars ascend the giant branch, the $\mathrm{Li}$ becomes diluted and destroyed as it mixes into deeper, hotter layers.

\subsection{Neutron-capture Elements}

Neutron-capture elements ( $\mathrm{Sr}$ to $\mathrm{U} ; 38 \leqslant Z \leqslant 92$ ) can originate from a variety of nucleosynthetic processes. It is thus not easy to disentangle the different sources and arrive at meaningful conclusions. The two major pathways for the production of these elements are the rapid ( $r$ ) process thought to occur in supernova explosions and the slow $(s)$ process thought to occur in AGB stars during stellar evolution. A detailed review of the importance of neutron-capture elements and their abundance in the MW halo can be found in Sneden et al. (2008).

\subsubsection{Strontium and Barium}

We measure the neutron-capture elements $\mathrm{Sr}$ and $\mathrm{Ba}$ in all of our stars. We find that both the $[\mathrm{Sr} / \mathrm{Fe}]$ and $[\mathrm{Ba} / \mathrm{Fe}]$ ratios are extremely depleted compared with the overall MW halo pattern. Low Ba abundances have also been found by Shetrone et al. (2001) for a few stars in the more luminous dSphs. It was suspected that $\mathrm{Sr}$ might be similarly depleted, but no $\mathrm{Sr}$ measurements exist because of the limited spectral coverage of those observations. We provide the first Sr measurements in any dwarf galaxy, ${ }^{12}$ and confirm that the $\mathrm{Sr}$ values are indeed at a similar level as $\mathrm{Ba}$. This result is not surprising since the nucleosynthetic origin of $\mathrm{Ba}$ and $\mathrm{Sr}$ is expected to be the same. In ComBer, $\mathrm{Sr}$ is almost constant at $[\mathrm{Sr} / \mathrm{Fe}] \sim-1.5$ over the metallicity range $-2.9<[\mathrm{Fe} / \mathrm{H}]<-2.3$. These values are very low, more than 1 dex below the bulk of the halo stars at the same Fe abundances (although as can be seen in Figures 11 and 17 , there are a few halo stars with similarly low levels). There is, however, a significant scatter of several dex (up to

\footnotetext{
${ }^{12}$ During the completion of this paper, Cohen \& Huang (2009) also obtained $\mathrm{Sr}$ abundances for their sample of Draco stars.
} 

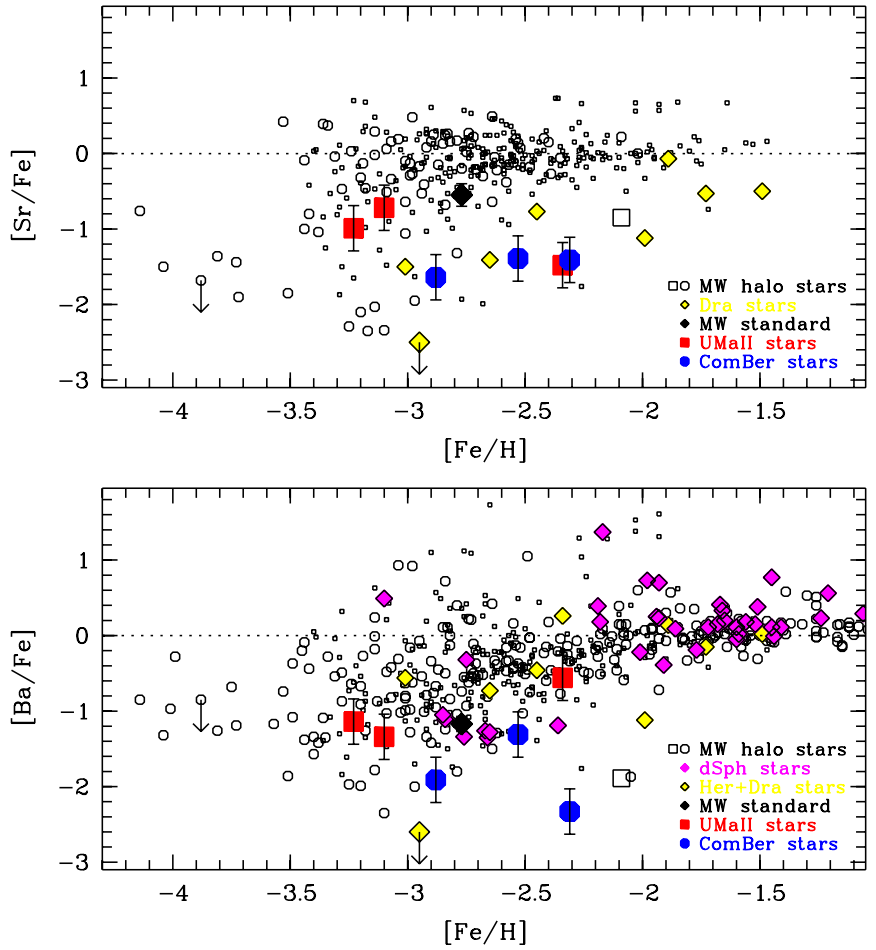

Figure 17. $[\mathrm{Sr} / \mathrm{Fe}]$ (top panel) and $[\mathrm{Ba} / \mathrm{Fe}]$ (bottom panel) abundance ratios as a function of $[\mathrm{Fe} / \mathrm{H}]$. Except for the upper limit in Dra119 (yellow diamond; Fulbright et al. 2004), these are the first $\mathrm{Sr}$ measurements in dwarf galaxies. All the ultra-faint dwarf galaxy $[\mathrm{Sr} / \mathrm{Fe}]$ and $[\mathrm{Ba} / \mathrm{Fe}]$ abundances (red squares: UMa II stars; blue circles: ComBer stars) are very low, at the lower end of the distribution of metal-poor halo stars (black squares and circles). Symbols are the same as in Figure 16.

(A color version of this figure is available in the online journal.)

$\sim 3$ dex) among halo stars at the lowest metallicities that is not well understood. François et al. (2007) find that the neutron-capture abundances generally decrease at the lowest $\mathrm{Fe}$ abundances, i.e., below $[\mathrm{Fe} / \mathrm{H}] \sim-3.0$. Furthermore, most of the stars with $[\mathrm{Fe} / \mathrm{H}] \lesssim-3.5$ have very low neutroncapture abundances (e.g., Sr and Ba; Norris et al. 2001), and this trend is also found in two of the three halo stars known with $[\mathrm{Fe} / \mathrm{H}]<-4.0$ (Christlieb et al. 2004; Norris et al. 2007).

In contrast to the low and uniform $\mathrm{Sr}$ abundances in ComBer, UMa II interestingly shows substantially higher $[\mathrm{Sr} / \mathrm{Fe}]$ ratios in the two stars with $[\mathrm{Fe} / \mathrm{H}] \sim-3.2([\mathrm{Sr} / \mathrm{Fe}] \sim-0.8)$ than in the star with $[\mathrm{Fe} / \mathrm{H}] \sim-2.3([\mathrm{Sr} / \mathrm{Fe}] \sim-1.3$, similar to the ComBer stars). The Sr values of the two extremely metal-poor stars fit well into the range seen in halo stars. Generally, the scatter of $\mathrm{Sr}$ abundances strongly increases with decreasing metallicity $[\mathrm{Fe} / \mathrm{H}]$, with more and more stars having $[\mathrm{Sr} / \mathrm{Fe}]$ abundances much lower than the solar value at low metallicities. Compared with the dwarf galaxy stars, HD 122563 is significantly Sr-enriched at $[\mathrm{Sr} / \mathrm{Fe}] \sim-0.6$, although this is still deficient by a factor of 4 relative to Fe compared to the Sun.

Even though all of our targets are deficient in $\mathrm{Ba}$, we also observe a pronounced scatter in the $[\mathrm{Ba} / \mathrm{Fe}]$ values. The constant trend of $\mathrm{Sr}$ abundances in ComBer is not followed in $\mathrm{Ba}$, with up to 1.5 dex of scatter (see Figure 17). All three ComBer stars have $\mathrm{Ba}$ abundances at or below the lower envelope of the halo stars; one star, ComBer-S1, is well below the entire Cayrel et al. (2004) halo sample, at $[\mathrm{Ba} / \mathrm{Fe}]=-2.33$. The $\mathrm{Ba} \lambda 4554 \AA$ line in this star is quite weak, as can be seen in Figure 2, although the detection is significant at the $3 \sigma$ level. Conservatively, one could regard this measurement as an upper limit, which would suggest an even more extreme underabundance of Ba. UMa II is somewhat different from ComBer, with its two most metalpoor stars also having $[\mathrm{Ba} / \mathrm{Fe}]$ ratios towards the low end of the halo distribution; the star at $[\mathrm{Fe} / \mathrm{H}] \sim-2.3$ has a much higher, almost solar $[\mathrm{Ba} / \mathrm{Fe}]$ ratio, although the abundances of this star may have a somewhat different origin (see Section 3.3.4).

Incomplete mixing could explain the significant differences among stars in each of our two dwarf galaxies in $\mathrm{Ba}$, and to some extent also in Sr. What is telling, though, is that with minor exceptions, all of the neutron-capture elements in the ultra-faint dwarf galaxies are at the same level as the lowest abundances found in the halo.

\subsubsection{Upper Limits}

For each of the stars we determined upper limits for $\mathrm{Y}, \mathrm{Zr}$, $\mathrm{La}, \mathrm{Ce}, \mathrm{Nd}, \mathrm{Sm}$, and $\mathrm{Eu}$ (with the exception of UMa II-S3, in which we were able to detect $\mathrm{Y}$ and La). They are listed in Tables 4, 6, and 7. The Y, Ce, and Eu limits are compared with halo abundances and limits in Figure 11. Generally, the limits indicate deficiencies in neutron-capture elements relative to the halo (particularly for our more metal-rich targets), consistent with the low $\mathrm{Sr}$ and $\mathrm{Ba}$ values. $[\mathrm{Y} / \mathrm{Fe}],[\mathrm{Ce} / \mathrm{Fe}]$, and $[\mathrm{Eu} / \mathrm{Fe}]$ in our more metal-rich stars are deficient by more than $\sim-0.5$ to -1 dex. Much higher $\mathrm{S} / \mathrm{N}$ data are needed to obtain more stringent limits and to explore whether all the neutron-capture elements in the ultra-faint dwarfs have depletion levels of $\sim-2$ dex with respect to the solar value.

\subsubsection{Origin of the Heavy Elements in the Ultra-faint Dwarf Galaxies}

We now use the observed neutron-capture abundances of our target stars to infer information about the different nucleosynthetic processes that played a role in the early history of the ultra-faint dwarf galaxies. At low metallicity, a major distinction can be made between the $r$ - and $s$-process signatures, indicating early SN II (pre-) enrichment or later mass transfer events, respectively.

Three of our stars have $\mathrm{Fe}$ abundances above the threshold value of $[\mathrm{Fe} / \mathrm{H}] \sim-2.6$ at which the $s$-process sets in for halo stars (Simmerer et al. 2004), while the other three have lower metallicities. In principle, this suggests that the $s$-process could be responsible for the observed neutron-capture abundance patterns of the higher-metallicity half of our sample. Indeed, one of our stars (UMa II-S3, with $[\mathrm{Fe} / \mathrm{H}] \sim-2.3$ ) may have a neutron-capture pattern consistent with an $s$-process signature, although its overall neutron-capture abundances are very low (usually the $s$-rich metal-poor stars exhibit [neutron-capture/Fe] values of $>0$ ). Since the neutron-capture abundances are so low it is not entirely clear whether the $s$-process of a previous generation of AGB stars could have enriched the gas cloud with $s$-material (e.g., through mass loss) from which our target formed, or if UMa II-S3 received this material from a binary companion. UMa II-S3 does, however, exhibits the typical radial velocity variations indicating binarity (see Section 3.3.4).

At low metallicities, the $r$-process is a promising candidate for the origin of the neutron-capture elements since it is associated with massive $\mathrm{SNe}$ II that are expected to have been present at very early times. The low $\mathrm{Fe}$ abundances $([\mathrm{Fe} / \mathrm{H}]<-2.6)$ of our three most metal-poor target stars thus indicate that the gas from which they formed was probably enriched through the $r$-process by SNe II from the previous generation of stars. As for the two more metal-rich stars (setting aside UMa II-S3 for the moment), their very low $\mathrm{Sr}$ and $\mathrm{Ba}$ abundances may suggest that they too originated from gas enriched by the $r$-process. 


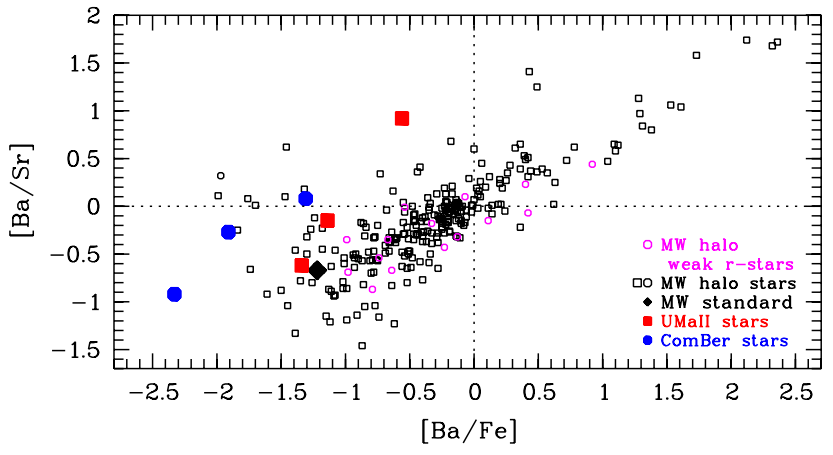

Figure 18. $[\mathrm{Ba} / \mathrm{Sr}]$ ratios for the program stars as a function of $[\mathrm{Ba} / \mathrm{Fe}]$ in comparison with other objects (black symbols) from Barklem et al. (2005); Lai et al. (2008) and François et al. (2007). Red squares indicate UMa II stars, blue circles show ComBer objects. The potentially weak $r$-process enriched HD 122563 is marked with an open black diamond. The full pink circles are Francois et al. stars classified as weak $r$-process stars by Izutani et al. (2009). At least two of our stars have $[\mathrm{Ba} / \mathrm{Sr}]$ values consistent with those of the Francois et al. weak- $r$ halo stars and HD 122563.

(A color version of this figure is available in the online journal.)

In recent years, it has been suggested that there are two components of the $r$-process, which produce somewhat different neutron-capture abundance distributions (Travaglio et al. 2004; Aoki et al. 2005; Otsuki et al. 2006). The weak $r$-process is thought to produce mainly the lighter neutron-capture elements $(Z<56)$ and little or no heavier neutron-capture elements, such as $\mathrm{Ba}$. The so-called main $r$-process, on the other hand, produces the full range of neutron-capture elements up to $Z=$ 92. The weak $r$-process has been suggested to occur in massive ( 20 $M_{\odot}$ ) core-collapse SNe (e.g., Wanajo \& Ishimaru 2006; Izutani et al. 2009), whereas the main component may occur in supernovae with lower mass $\left(8-10 M_{\odot}\right)$ progenitors (Qian \& Wasserburg 2003). The two different signatures are principally observable in suitable stars, ${ }^{13}$ and, for example, HD 122563 has been suspected of exhibiting a weak $r$-process signature because the abundance of $\mathrm{Ba}$ and heavier elements are depleted (Honda et al. 2006). From examining the $[\mathrm{Ba} / \mathrm{Sr}]$ ratio, which reflects the relative contributions of the two processes, clues can be obtained as to the potential origin of the overall abundance pattern. If the main $r$-process were at work, higher overall levels of $\mathrm{Sr}$ and $\mathrm{Ba}$ would be expected (e.g., Honda et al. 2007).

The extremely low $\mathrm{Ba}$ abundances (and somewhat higher Sr levels) we observe are thus suggestive of the weak $r$-process as the most likely nucleosynthetic origin for the neutron-capture elements in UMa II and ComBer. In Figure 18, we compare the $[\mathrm{Ba} / \mathrm{Sr}]$ ratios of our stars with the MW halo ratios. We find that our targets have similar abundance ratios to HD 122563, indicating that the explosions of very massive stars might have provided the early chemical enrichment in both UMa II and ComBer. This would be in accord with the halo-typical enhancements of $\alpha$-elements that also originate from nucleosynthesis in massive stars (e.g., McWilliam et al. 1995). The production of $\mathrm{Fe}$ is decoupled from that of the neutron-capture elements (e.g., Sneden et al. 2008), so one would still have to explain the spread in $\mathrm{Fe}$ found in our sample to arrive at a global explanation for the chemical abundance patterns of these systems. Fe abundances around $[\mathrm{Fe} / \mathrm{H}] \sim-2.5$ in our sample suggest that at least a few supernovae were responsible for the early enrichment of these galaxies. Perhaps the supernovae that produced most of the Fe in

\footnotetext{
13 Objects that exhibit strong overabundances in neutron-capture elements have low effective temperature, and are bright enough for high-resolution, high $\mathrm{S} / \mathrm{N}$ spectra to be acquired.
}

UMa II and ComBer had (slightly) different progenitor masses, so that several generations of short-lived stars contributed different (groups of) elements to the ISM of these systems. If only a fraction (or even just one) of those SNe hosted nucleosynthesis through the weak- $r$ process that could explain the extremely low and roughly constant levels of $\mathrm{Sr}$ and $\mathrm{Ba}$ as a function of $[\mathrm{Fe} / \mathrm{H}]$ in these systems.

The two Her stars observed by Koch et al. (2008b) have non-detectable $\mathrm{Ba}$ lines with upper limits of $[\mathrm{Ba} / \mathrm{Fe}]<-2.1$. Koch et al. speculated that massive $\left(\sim 35 M_{\odot}\right)$ stars were responsible for the observed light element abundance pattern. However, this hypothesis requires that those massive stars did not produce significant quantities of $\mathrm{Ba}$ (or perhaps any $\mathrm{Ba}$ at all), again suggestive of a weak- $r$ signature. Given such low $\mathrm{Ba}$ abundances, the weak $r$-process would produce a low $\mathrm{Ba} / \mathrm{Sr}$ ratio of $[\mathrm{Ba} / \mathrm{Sr}] \sim-1$ (Figure 18). Thus, although Koch et al. (2008b) did not observe Sr, we predict that the Sr abundances in the Her stars should be relatively large and enhanced with respect to $\mathrm{Ba}$, and hence potentially measurable. Observations of neutron-capture elements for additional stars in this and other dwarf galaxies will shed light on the weak- $r$ hypothesis and will help disentangle the somewhat peculiar chemical nature of Her, as well as that of the broader ultra-faint dwarf galaxy population.

\subsubsection{UMa II-S3-An s-rich Binary Star System?}

Since we observed UMa II-S3 with an additional, bluer spectrograph setting, we were able to obtain abundances of several additional elements that have strong absorption lines blueward of $4150 \AA$. Due to the lower $\mathrm{S} / \mathrm{N}$ in this region $(\sim 10$ at $4000 \AA$ ), all these abundances have slightly larger uncertainties. We derive an $\mathrm{Al}$ abundance of $[\mathrm{Al} / \mathrm{Fe}]=-0.34 \pm 0.3$ from the two lines at $3944 \AA$ and $3961 \AA$. This value agrees very well with Cayrel et al. (2004) halo stars. The Si line at $4102 \AA$ is very strong and yielded $[\mathrm{Si} / \mathrm{Fe}]=0.91 \pm 0.3$. This is slightly above the trend of the Cayrel et al. stars, although our value is somewhat uncertain because the low $\mathrm{S} / \mathrm{N}$ data hampered the continuum placement. Four Co lines could also be detected in the bluer setting. Our value of $[\mathrm{Co} / \mathrm{Fe}]=-0.09 \pm 0.1$ agrees well with the other halo star abundances. We also co-added this bluer spectrum with the spectrum taken with the "standard" setting. This yielded the detection of $\mathrm{Zr}$ at $\lambda 4209$. The $\lambda 4317$ line was not detected but the upper limit is consistent with the $\mathrm{Zr}$ abundance of $[\mathrm{Zr} / \mathrm{Fe}]=-0.60 \pm 0.3$. The Sr line at $4077 \AA$ is very strong, and its abundance is consistent with that of the $4215 \AA$ line. Because of the higher $\mathrm{S} / \mathrm{N}$, we adopt the $\mathrm{Sr}$ abundance of the $\lambda 4215$ line. Eu is still not detected in the combined spectrum. The lines at $4129 \AA$ and $4205 \AA$ have similar upper limits (note that the $\mathrm{S} / \mathrm{N}$ at $4200 \AA$ is slightly larger $[\mathrm{S} / \mathrm{N}$ $\sim 17]$ than at $4100 \AA[\sim 13])$. The limit of [Eu/Fe] $<-0.57$ is also consistent with the $\lambda 4435$ line, which is mostly a blend of $\mathrm{Ca}$ and Eu.

In summary, we detect not only $\mathrm{Sr}$ and $\mathrm{Ba}$ in UMa II-S3 but also the neutron-capture elements $\mathrm{Zr}$, Y, and La. The latter two were detected in the redder spectrum. In Figure 19, we compare our abundances and upper limits of the neutron-capture elements to those predicted from the scaled solar $r$-and $s$-process patterns (Burris et al. 2000) to obtain clues as to where the neutroncapture elements originate. As can be seen in the figure, our abundances do not agree with the $r$-process pattern (particularly the upper limits for $\mathrm{Ce}, \mathrm{Nd}, \mathrm{Sm}$, and $\mathrm{Eu}$ ), but they might be consistent with the $s$-process pattern.

In the MW halo, $s$-process-rich metal-poor stars are found down to metallicities of $[\mathrm{Fe} / \mathrm{H}] \sim-2.6$ (Simmerer et al. 2004). 


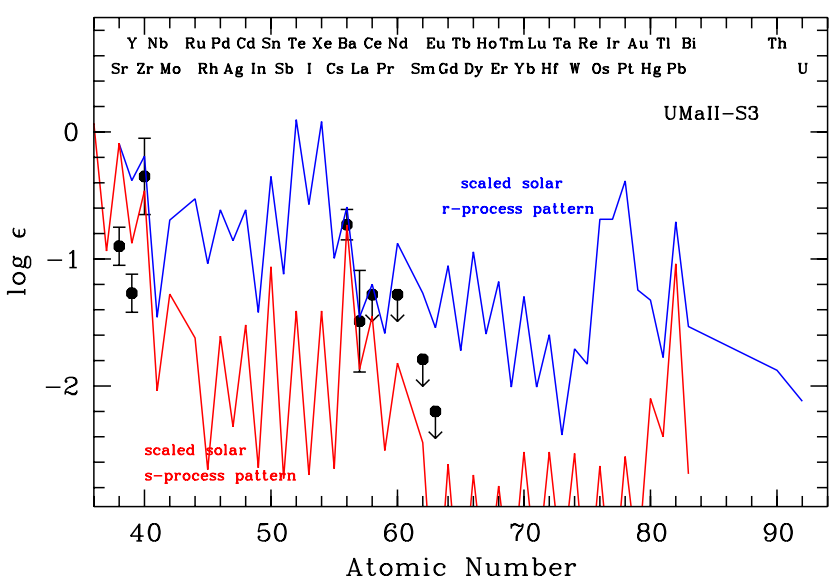

Figure 19. Neutron-capture abundances and upper limits for UMa II-S3 overplotted with the scaled solar $r$ - and $s$-process patterns (scaled to Ba). There is no agreement with the $r$-process pattern. The chemical signature of UMa II-S3 may, however, be consistent with the $s$-process.

(A color version of this figure is available in the online journal.)

These stars experienced mass transfer from a slightly more massive $\left(\sim 1-5 M_{\odot}\right)$ companion as it passed through the AGB phase. During the mass transfer event, these $s$-elements, together with dredged-up $\mathrm{C}$, are donated to the lower mass companion that we observe today as a metal-poor giant. Thus, the $s$-process enrichment is usually accompanied by an overabundance in $\mathrm{C}$. However, in the case of UMa II-S3, the C-enrichment (often $[\mathrm{C} / \mathrm{Fe}]>+1.0)$ is missing. Also, the overall neutron-capture abundances in the star are extremely low, in contrast to that of the usual $s$-material overabundances of $[s / \mathrm{Fe}]>0$ observed in known $s$-enriched metal-poor stars. This fact is rather puzzling and may potentially challenge the conjecture that UMa II-S3 is an $s$-rich star. If the star did not receive the $s$-rich material from a companion, then maybe a previous generation of AGB stars producing $s$-elements could have enriched the gas cloud, e.g., through extensive mass loss, from which UMa II-S3 later formed. Nevertheless, radial velocity variations are observed for UMa II-S3 that indicate binarity and offer some observational support for the mass transfer event. Concerning the binary nature, we have three radial velocity measurements that demonstrate that this star is indeed in a binary system. We list two of those values in Table 2 , showing a velocity change of more than $15 \mathrm{~km} \mathrm{~s}^{-1}$ over the course of a year. Note that the DEIMOS spectrum was taken on 2007 February 13, with $\mathrm{S} / \mathrm{N}=$ 116. Its lower resolution of $R=6000$ results in a somewhat larger uncertainty. The third measurement from 2007 November 5 is $-118.4 \pm 0.5 \mathrm{~km} \mathrm{~s}^{-1}$. It was obtained from an $R=50,000$, low $\mathrm{S} / \mathrm{N}$ HIRES early test spectrum. Future radial velocity measurements are required to establish the orbital parameters of this object.

An unusual chemical history for this star may also explain why other light elements deviate from the abundances of our other program stars as well as those of the halo stars. It is somewhat $\mathrm{Mg}$ - and Ca-rich $([\mathrm{Mg} / \mathrm{Fe}] \sim+0.8$ and $[\mathrm{Ca} / \mathrm{Fe}] \sim$ $+0.5)$, and thus lies slightly above the general trends seen in our data set (see Figure 16). The $\mathrm{Ba} / \mathrm{Fe}$ ratio is much higher in UMa II-S3 compared with the other stars in the ultra-faint dwarfs, and almost as high as the $\mathrm{Ba}$ abundance found in the more luminous dSphs (Shetrone et al. 2003, 2001) at higher Fe abundances. Interestingly, this star seems to bridge the gap between our low Ba stars (and also Dra 119 and Her) with the stars in the brighter dSphs. This could also suggest that there is a smooth metallicity (Fe-)dependent transition. At the lowest metallicities, $\mathrm{Ba}$ (and also $\mathrm{Sr}$ ) seems to trail the lower envelope of the halo star abundances, as is also true for the two most metal-deficient stars from Shetrone et al. (2003). Above $[\mathrm{Fe} / \mathrm{H}] \sim-2.3$, the halo abundance scatter considerably tightens and the stars in the more luminous dSphs suddenly appear all in agreement with the halo abundances. Clearly, more stars at $[\mathrm{Fe} / \mathrm{H}]<-2.0$ are required to assess in greater detail whether the neutron-capture elements in dwarf galaxies generally lie below the halo, or if UMa II-S3 shows an unusual, atypical chemical pattern. Finally, we stress that any $s$-process mass transfer onto UMa II-S3 should not have affected its light element ( $\mathrm{Na}$ to $\mathrm{Zn}$ ) signature, so this star can still be employed for tracing the chemical composition of the ISM at the time of its formation.

\section{CHEMICAL HISTORY OF THE ULTRA-FAINT DWARF GALAXIES}

Based on the individual abundances of their member stars, we now discuss the implications of our results for the early chemical history and star formation history of UMa II and ComBer. We also consider how the ultra-faint dwarf galaxies may fit into the broader picture of hierarchical galaxy formation.

\subsection{Existence of Extremely Metal-poor Stars}

Kirby et al. (2008) presented the first evidence for the existence of extremely metal-poor stars with $[\mathrm{Fe} / \mathrm{H}]<-3$ in any dwarf galaxy, identifying 15 such stars in the ultrafaint dwarf galaxies. After obtaining high-resolution spectra of two of these targets with the lowest metallicities (UMa II-S1 and UMa II-S2), and a comprehensive uncertainty analysis, the measured $\mathrm{Fe}$ abundances are $[\mathrm{Fe} / \mathrm{H}]=-3.1$ and $[\mathrm{Fe} / \mathrm{H}]=$ -3.2 , respectively (where $[\mathrm{Fe} / \mathrm{H}]=[\mathrm{Fe} \mathrm{I} / \mathrm{H}]=[\mathrm{Fe} \mathrm{II} / \mathrm{H}])$. The total uncertainties as listed in Table 8 are 0.3 dex for Fe I and 0.2 dex for Fe II for such giants. From the model atmosphere comparison in Section 2.5.3, we furthermore find that the inclusion of scattering would lower the measured Fe abundances by 0.1 dex. In summary, these assessments strongly suggest that within the uncertainties, the two stars cannot be significantly above $[\mathrm{Fe} / \mathrm{H}]=-3.0$, and are in fact true extremely metal-poor stars. Our results thus confirm the conclusions of Kirby et al. and suggest that their method is indeed suitable for identifying extremely metal-poor stars.

While the sample of stars we have investigated is small, our HIRES targets were selected only on the basis of their apparent magnitude; therefore, finding two stars with $[\mathrm{Fe} / \mathrm{H}]<-3$ out of just six targets hints that a significant fraction of the stars in the faintest dwarf galaxies may have had extremely low metallicities. Previous studies using both the $\mathrm{Ca}$ triplet and high-resolution spectroscopy in the classical dSphs had only identified a handful of stars below $[\mathrm{Fe} / \mathrm{H}]=-2.7$ (e.g., Fulbright et al. 2004; Sadakane et al. 2004; Cohen \& Huang 2009; Aoki et al. 2009), with the vast majority at metallicities above $[\mathrm{Fe} / \mathrm{H}]=-2$ (e.g., Shetrone et al. 2001, 2003; Koch et al. 2008a). However, since the total number of stars observed at high resolution across all of the brighter dSphs is just $\sim 50$, it is not yet clear whether the absence of extremely metal-poor stars in those galaxies reflects a true deficit or a bias in the $\mathrm{Ca}$ triplet $[\mathrm{Fe} / \mathrm{H}]$ values at low metallicities.

\subsection{Large Internal Abundance Spreads}

Earlier medium-resolution spectra showed that each of the ultra-faint dwarf galaxies contain stars with a range of $\mathrm{Fe}$ 
abundances covering $\sim 0.5$ dex rather than a single stellar population (Martin et al. 2007; Simon \& Geha 2007; Kirby et al. 2008; Norris et al. 2008). Our measurements conclusively demonstrate that these abundance spreads are real—even with only three stars in each galaxy, our targets span a range of 0.9 dex in UMa II and 0.6 dex in ComBer.

There are several ways to produce the internal abundance spreads observed in these extremely low luminosity galaxies. (1) If the stars were formed in multiple smaller progenitor systems that later merged to become the dwarf galaxy we see today, then it would be natural for the ISM in each of the proto-dwarf galaxies to have had a different metallicity. (2) If the stars formed in situ (i.e., in the main halo that became the present-day dwarf galaxy), the star-forming gas may have been incompletely mixed either as a result of asymmetric supernova explosions or rapid star formation before mixing could occur. (3) Finally, if the young UMa II and ComBer were able to hold onto their gas for an extended period of time (or re-accrete enough gas later to produce multiple epochs of star formation), the stars formed at later times would have higher metallicities because of the continued chemical evolution of the ISM.

One way to separate these possibilities may be high signal-tonoise photometry in the main-sequence turnoff region to determine whether there is a significant age spread among the member stars. Obtaining spectra of more metal-rich stars in these two dwarf galaxies would also be useful to see if the $[\alpha / \mathrm{Fe}]$ ratios indicate any contribution from Type Ia supernovae. Our data are consistent with a constant $[\alpha / \mathrm{Fe}]$ as a function of $[\mathrm{Fe} / \mathrm{H}]$, but measurements at $[\mathrm{Fe} / \mathrm{H}] \geqslant-2$ are needed to reveal whether the turn-down in $[\alpha / \mathrm{Fe}]$ seen in systems with extended star formation histories (e.g., the classical dSphs and the MW halo) is present in the ultra-faint dwarf galaxies as well.

\subsection{Comparison to the MW Halo and the More Luminous dSphs}

In contrast to previous studies of the more luminous dSphs (e.g., Shetrone et al. 2001, 2003; Tolstoy et al. 2003; Sadakane et al. 2004; ${ }^{14}$ Venn et al. 2004; Geisler et al. 2007; see Tolstoy et al. 2009 for a recent review on this topic), the abundances found in our two ultra-faint dwarf galaxies generally agree with those of MW halo stars (see Figure 10). The observed abundance pattern includes the halo-typical abundance offset of $\sim 0.4$ dex among the $\alpha$-elements that results from ISM enrichment by massive SN II (e.g., Woosley \& Weaver 1995). This agreement may point to an initial mass function similar to the one that produced the halo star abundance pattern at early times, and furthermore to a significant contribution from massive stars to the early enrichment of the ultra-faint systems. This would be consistent with the overall low metallicity of all of the newly discovered dwarf galaxies (Kirby et al. 2008), and feedback from those supernovae might be responsible for suppressing star formation in these systems. The more luminous dSphs have significantly higher average metallicities than the ultrafaint dwarf galaxies. Their lower, more solar-like $\alpha$-element ratios (see Figure 16) clearly point to a major contribution of Fe from Type Ia supernovae, and potentially a mass function shifted to lower masses. Tolstoy et al. (2003) pointed out that small systems may naturally have an initial mass function with a suppressed high-mass end as a result of the difficulty of forming

\footnotetext{
14 Their lowest metallicity star shows a somewhat similar chemical pattern to that found in the present study indicating SN II enrichment. The large Fe spread in UMi of $\sim 1$ dex is comparable to what is found in UMa II, although reaching higher $\mathrm{Fe}$ values and reflecting enrichment by SNe Ia as well as massive stars.
}

large molecular clouds (and therefore very massive stars) in such low-density environments.

Our two most metal-poor stars $($ at $[\mathrm{Fe} / \mathrm{H}]<-3.0)$ are enriched in carbon. In the halo, there is an increasing trend of $\mathrm{C}$ enhancement with decreasing metallicity. Although our sample is quite small, this may be another signature that the ultra-faint dwarf galaxies share with the MW halo. Overall, C excesses in the most metal-poor stars point toward the important role of $\mathrm{C}$ in the early universe and as a potential cooling agent of the primordial ISM (Beers \& Christlieb 2005; Aoki et al. 2007a; Frebel et al. 2007a). The presence of C-rich extremely metalpoor stars is consistent with the assumption that the massive stars in a system produced the $\mathrm{C}$ either during stellar evolution or during their supernova explosions.

It is less clear whether the stellar abundances of neutroncapture elements in the ultra-faint dwarf galaxies are consistent with those in the MW halo and more luminous dSphs. The observed $\mathrm{Ba}$ and $\mathrm{Sr}$ abundances are near the low end of the neutroncapture-to-iron ratios seen in halo stars, whereas the abundances found in the more luminous dSphs (at higher metallicity) agree rather well with those found in the halo. Low neutron-capture abundances are also found in Hercules (Koch et al. 2008b), two stars in the more luminous dSph Draco (Fulbright et al. 2004; Shetrone et al. 2001), one star in Sextans (Shetrone et al. 2001), and one in Sadakane et al. (2004). An important question therefore arises as to how a system can enrich itself significantly with iron-peak elements, but produce very little to no neutron-capture elements in a consistent way over a long period of time (up to relatively high metallicities of $[\mathrm{Fe} / \mathrm{H}] \sim-2.3$ ). Our observations underscore that the production of these element groups has to be strongly decoupled, as already evidenced by halo star abundance patterns (e.g., Sneden et al. 2008). This question can only be adequately addressed with a much larger sample of stars in the ultrafaint dwarf galaxies, and detections of heavier neutron-capture species would also be helpful. In the meantime, we speculate in Section 3.3.3 that the neutron-capture elements in the ultra-faint dwarfs were produced through the weak $r$-process in stars more massive than $\sim 8-10 M_{\odot}$ (which is the mass range of the main $r$-process, see Section 3.3). Such massive stars $\left(\sim 20 M_{\odot}\right.$; e.g., Wanajo \& Ishimaru 2006) might also have been responsible for the halo-like levels of $\alpha$-elements observed in our stars. Izutani et al. (2009) recently calculated weak $r$-nucleosynthesis yields and found that energetic hypernovae with $20 M_{\odot}$ are needed to reproduce the supposed weak $r$-abundance pattern in metal-poor halo stars. This agrees with findings by Nomoto et al. (2006), who explained the abundances of the Cayrel et al. (2004) stars with their hypernova models. Since our stars are very similar to the Cayrel et al. stars (see Figure 10), this is a plausible explanation for the chemical signature of stars with $[\mathrm{Fe} / \mathrm{H}] \lesssim-2.5$. Because our abundance measurements point to the weak $r$-process, operating in massive stars, as the source of the heavy elements in the ultra-faint dwarfs, it might even be the case that the population of lower mass $\left(\sim 8-10 M_{\odot}\right)$ SN II progenitors was suppressed at early times in these objects. In summary, the low levels of neutron-capture elements may simply reflect a local environment that was driven by a particular mass function of its stars.

These conclusions regarding the source of the $r$-process enrichment differ strongly from the conclusions of previous studies. Venn et al. (2004) suggested that in the more luminous $\mathrm{dSphs}$ there may be a lack of hypernovae and Tolstoy et al. (2003) suggested that the IMF was shifted to lower mass stars. However, given the different abundance patterns of the ultrafaint dwarfs and the more luminous dSphs this contradiction 
may not be surprising. Leaving aside all the uncertainties in nucleosynthesis processes and small-number statistics of our small samples, the abundance data themselves (see Figures 16 and 17) reveal different enrichment histories for the ultra-faint dwarfs and their brighter counterparts. This contrast between the abundance patterns seen in high- and low-luminosity dwarf galaxies demonstrates again that the ultra-faint dwarfs cannot simply be tidally stripped versions of the classical dSphs (Peñarrubia et al. 2008; Kirby et al. 2008; Geha et al. 2009). Spectroscopy of more stars in the ultra-faint dwarf galaxies as well as in the more luminous dSphs is needed to establish more firmly exactly how the MW's population of dwarf galaxies evolved and to what extent their chemical abundances are correlated strictly with luminosity. As Kirby et al. (2008) showed, there is a strong correlation of $\mathrm{Fe}$ abundance with luminosity, but for other elements this picture may be different.

While we were in the process of completing this paper, two new studies of brighter dSphs were published. Cohen \& Huang (2009) presented a high-resolution abundance analysis of 8 stars in the classical dSph Draco. Their stars span the range from $-3.0 \leqslant[\mathrm{Fe} / \mathrm{H}] \leqslant-1.5$, with one star at $[\mathrm{Fe} / \mathrm{H}]=-3.0$. At the low-metallicity end they find the abundances of several elements to be in agreement with those of halo stars, but at higher metallicities, deviations are found. The $\alpha$-abundances ( $\mathrm{Ca}$ and $\mathrm{Ti}$ ), however, are depleted relative to the MW halo, as has been found in the higher metallicity stars in the luminous dwarf galaxies. The $\mathrm{Sr}$ abundances at the low-metallicity end are similarly low as found in the ultra-faint dwarfs, but rise up to solar at higher metallicities. The Ba values follow a similar trend but at a slightly more elevated level.

In Sextans, Aoki et al. (2009) found one star with $[\mathrm{Fe} / \mathrm{H}]=$ -3.1 and five with $-2.9<[\mathrm{Fe} / \mathrm{H}]<-2.7$. Their most metalpoor star seems to mostly follow the Galactic halo $[\mathrm{X} / \mathrm{Fe}]$ trends in the same fashion as the objects presented in this study. However, the $\mathrm{Ba} / \mathrm{Fe}$ ratio in their $[\mathrm{Fe} / \mathrm{H}]=-3.1$ star is high $([\mathrm{Ba} / \mathrm{Fe}]=0.5)$, which is different from what has been found in this study. On the other hand, their slightly more metal-rich stars show depletions similar to those generally found in the luminous dwarf galaxies $($ at $[\mathrm{Fe} / \mathrm{H}] \gtrsim-2.5)$, as well as the low $\mathrm{Ba}$ abundances that have been found for our UMa II and ComBer stars.

These new results suggest that there may be a metallicity $([\mathrm{Fe} / \mathrm{H}])$ dependence for elemental ratios to be more halo-like at metallicities below $[\mathrm{Fe} / \mathrm{H}]=-2.5$ in all dwarf galaxies, not just the ultra-faint ones. However, the body of stellar data in the luminous dwarf galaxies may not yet be sufficient to derive strong conclusions about their most metal-poor stars, especially in light of the fact that counter examples are also evident (e.g., low $\mathrm{Ca}$ and $\mathrm{Ti}$ at $[\mathrm{Fe} / \mathrm{H}]=-3.0$; Cohen \& Huang 2009).

\subsection{Comparison to Globular Cluster Abundances}

We have so far found evidence that the chemical signatures of stars in the ultra-faint dwarf galaxies closely resemble those of halo field stars. An outstanding question, then, is if there are also similarities to globular cluster stars. It has been debated, and so far been excluded (e.g., Simon \& Geha 2007), that the ultra-faint dwarf galaxies might be globular clusters instead of dark matter-dominated galaxies. Adding chemical information to this discussion may provide further constraints on the origin of these dim systems. A first and obvious difference between the dwarf galaxy stars and the globular cluster members is the large $\mathrm{Fe}$ abundance spread compared with the mono-metallic populations in globular clusters. Furthermore, our Fe values are generally lower than those of the most metal-poor globular cluster (Harris 1997). This behavior was already pointed out by Simon \& Geha (2007) and Kirby et al. (2008) for the ultra-faint dwarf galaxies and is not limited to the two systems studied here.

Aside from the very different behavior in $\mathrm{Fe}$ abundances, there exist a number of additional characteristic globular cluster abundance patterns involving low $\mathrm{C}$ and $\mathrm{O}$, and high $\mathrm{N}, \mathrm{Na}$, and $\mathrm{Al}$ that we can investigate in the ultra-faint dwarf galaxies.

For the low $[\mathrm{C} / \mathrm{Fe}]$ globular cluster giants, it has been shown (e.g., Shetrone et al. 1999) that deep mixing events on the RGB and prior nucleosynthesis could both be responsible for the low $\mathrm{C}$ values, but these mechanisms are difficult to distinguish even in extreme cases (e.g., Sneden et al. 2004). It is thus unclear if the level of depletion in cool giants arises from deep mixing where material in which $\mathrm{C}$ has been converted to $\mathrm{N}$ is dredged up to the surface or if the stars were simply born from $\mathrm{C}$-poor material since the $\mathrm{C}-\mathrm{N}$ anticorrelation is found down to main-sequence stars in some clusters (Harbeck et al. 2003). Since we have no $\mathrm{N}$ and $\mathrm{O}$ measurements, we are unfortunately not able to address in detail whether the low $\mathrm{C}$ abundances observed in our dwarf galaxy stars arise from carbon-depleted material. Irrespective of considering our stars as globular cluster or halo star analogs, their evolutionary status alone suggests that some mixing may already have taken place and the currently observed subsolar levels of two of our stars are in agreement with halo stars of similar evolutionary status (and which are assumed not to have formed from C-depleted material). This suggests that our stars did not form from particularly C-poor gas as some clusters have. The only firm conclusion that is possible from the available $\mathrm{C}$ abundances alone is that our two stars with the highest $\mathrm{C}$ values $([\mathrm{C} / \mathrm{Fe}] \sim+0.5$ and $\sim+0.8$; both in UMa II) appear to be more carbon-rich than is typical in clusters. And even the two stars with $[\mathrm{C} / \mathrm{Fe}] \sim 0.0$ (both in ComBer) do not necessarily indicate either substantial mixing or being born from very $\mathrm{C}$-depleted material. We thus conclude that at present there is no strong indication that the $\mathrm{C}$ abundances in dwarf galaxies behave similarly to those in globular clusters (where the C-depletion is not due to deep mixing alone).

For the following discussion, we correct our $\mathrm{Na}$ abundances for non-LTE effects for the comparison with the results of Sneden et al. (2004). We thereby adopt the same corrections (Gratton et al. 1999) as Sneden et al. For completeness, we remind the reader that in the earlier comparison with the Cayrel et al. halo sample, the uncorrected LTE Na values were used.

$\mathrm{Na}-\mathrm{O}$ correlation. While we have no $\mathrm{O}$ abundances available, we can nevertheless consider the $\mathrm{Na}$ abundance distribution of globular cluster stars and halo field stars. In clusters, it has been found that the stars closest to the RGB tip have the highest $\mathrm{Na}$ abundances (up to $[\mathrm{Na} / \mathrm{Fe}] \sim 1.0$; Sneden et al. 2004 and references therein). This may originate from protoncapture and thus be a sign of deep mixing. The halo appears to lack such extreme equivalents. If the mixing scenario is correct, we should not find such extreme $\mathrm{Na}$ abundances in our sample since our targets do not sit at the tip of the giant branch. Indeed, our corrected $\mathrm{Na} / \mathrm{Fe}$ values span the range from -0.45 to +0.45 dex. There is an overlap in $\mathrm{Na}$ abundances between the ultra-faint dwarf galaxies and globular clusters, although the former systems also have some stars with $\mathrm{Na}$ abundance lower than those ever seen in clusters. Regarding the one star with $[\mathrm{Na} / \mathrm{Fe}] \sim 0.45$ (ComBer-S3), it is unclear whether this could be interpreted as some globular cluster signature because the star with the lowest $\mathrm{Na},[\mathrm{Na} / \mathrm{Fe}] \sim-0.7$, is in the same dwarf 
galaxy (ComBer II-S1). Such low abundances are not found in clusters.

$\mathrm{Na}$-Al correlation. We have one Al detection available, yielding a low abundance $([\mathrm{Al} / \mathrm{Fe}]=-0.34)$. Together with the corresponding $\mathrm{Na}$ abundance, UMa II-S3 does not lay on the $\mathrm{Na}-\mathrm{Al}$ correlation for clusters presented in Sneden et al. (2004). The lack of additional $\mathrm{Al}$ measurements precludes a strong conclusion, although the one detection further supports the idea that UMa II shows no distinct sign for a cluster chemical history.

$\mathrm{Na}-\mathrm{Mg}$ correlation. All six of our targets (as well as HD 122563) lie in the high-Mg, low-to-intermediate Na range of Figures 13 and 14 of Sneden et al. (2004). These authors show that this region is sparsely and in some cases not at all populated by cluster members. We interpret this as an additional clue to the non-cluster-like chemical origin of our targets.

In summary, the significant difference of $\mathrm{Fe}$ abundances and spreads between clusters and ultra-faint dwarfs already suggested that our stars are not associated with globular clusters or globular cluster-like nucleosynthesis histories and events. Based on our examination of the abundance correlations typically seen in globular clusters, we do not find clear signs for any correspondence between the abundance patterns in the ultra-faint dwarfs and those of globular clusters. We note, though, and given our limited sample size, more observations of ultra-faint dwarf galaxy stars could be helpful to further address this issue.

\subsection{Building Up the Metal-poor Halo}

The similarity of the abundance pattern we find in UMa II and ComBer (Sections 3.2 and 4.3) to the well-known abundance pattern seen in very low-metallicity MW halo stars suggests the possibility of a common origin of the two populations. Could the bulk of the metal-poor end of the halo metallicity distribution have been formed in galaxies similar to the ultra-faint dwarfs?

Kirby et al. (2008) demonstrated that integrated over the ultrafaint dwarf galaxies with $-8 \lesssim M_{V} \lesssim-4, \sim 5 \%$ of the stars have metallicities $[\mathrm{Fe} / \mathrm{H}] \leqslant-3$. Our results suggest that for the lowest luminosity galaxies the fraction of extremely metal-poor stars may be even higher, but for galaxies with stellar masses of $\sim 10^{4} M_{\odot}$ (Martin et al. 2008) $\sim 5 \%$ should be a representative value. Every such galaxy that has been destroyed by the MW therefore must have added $\sim 500 M_{\odot}$ of extremely metal-poor stars to the halo.

The fraction of such low-metallicity stars in the brighter $\mathrm{dSphs}$ is not currently known, since no stars with $[\mathrm{Fe} / \mathrm{H}] \leqslant-3$ have been detected in those galaxies, but the large number of stars observed without finding any extremely metal-poor stars (e.g., Helmi et al. 2006; Koch et al. 2006) indicates that $\sim 0.1 \%$ would be a reasonable assumption. Given a typical stellar mass of $\sim 10^{6} M_{\odot}$ (similar to Sculptor, Carina, or Sextans), the destruction of a classical dSph would add $\sim 1000 M_{\odot}$ of extremely metal-poor stars to the halo. Current estimates are that the MW's satellite population today includes $\sim 5$ times as many ultra-faint dwarf galaxies as classical dSphs (Simon \& Geha 2007; Koposov 2008; Tollerud et al. 2008). If the population of dwarfs that has been cannibalized over the lifetime of the MW had a similar luminosity function, then the progenitors of the ultra-faint dwarfs would have provided $\sim 2.5$ times as many extremely metal-poor stars as the more luminous dSphs. Thus, even though the ultra-faint dwarf galaxies simply do not contain enough stars to contribute significantly to the overall stellar mass of the MW halo, it is plausible that such galaxies could be the source of a large fraction of the most metal-poor halo stars.

\section{6. $\Lambda C D M$ Simulations}

We now consider whether the observational results summarized in Sections 4.1 to 4.3 are consistent with expectations from the predictions of $\Lambda \mathrm{CDM}$ plus galaxy formation models, where the MW's halo is largely composed of stars stripped from infalling dwarf galaxies.

The most detailed set of predictions have come from the semianalytic plus $N$-body model described in Bullock \& Johnston (2005) and Robertson et al. (2005; see also the related papers by Font et al. 2006 and Johnston et al. 2008.) The discrepant $\alpha$ abundance patterns of stars in the classical dSphs and the MW's field halo population are consistent with this model, owing to two important differences between the surviving MW dwarf galaxies and those that were destroyed to build the stellar halo. First, the stars in the halo came from dwarf galaxies accreted on average $9 \mathrm{Gyr}$ in the past, whereas the dwarf galaxies surviving today were accreted on average 5 Gyr ago. Second, the majority of stars in the halo were formed in dwarf galaxies substantially more massive $\left(M \sim 5 \times 10^{10} M_{\odot}\right)$ than the dwarf satellites surviving around the MW until today $\left(M \sim 5 \times 10^{8} M_{\odot}\right)$.

This model is broadly consistent with our observations that the ultra-faints have (1) a high frequency of extremely metalpoor stars (see also Kirby et al. 2008), and (2) abundance patterns generally consistent with those of MW field halo stars. The chemical evolution model of Robertson et al. (2005), Font et al. (2006), and Johnston et al. (2008) predicts that although massive dwarf galaxies are the source of the vast majority of the mass in the stellar halo, low-luminosity, metal-poor, galaxies accreted at early times contribute some stars to the metal-poor stellar halo and have $[\alpha / \mathrm{Fe}]$ abundances similar to those observed in low-metallicity halo stars. Robertson et al. (2005) emphasize that the lowest $[\mathrm{Fe} / \mathrm{H}]$ stars even in massive dwarf galaxies (such as the Magellanic Clouds, and the dSphs predicted to build up the majority of the halo) should also exhibit similar abundance patterns to the most metal-poor halo stars, although this prediction has not yet been tested by observations.

In an alternative approach, Prantzos (2008) uses an analytic model to predict the MDF of the MW halo from a set of satellites with stellar masses ranging from $2 \times 10^{6}$ to $2 \times 10^{8} M_{\odot}$. In his model, the shape of the MDF is also a strong function of satellite mass, with lower stellar mass satellites having a much higher fraction of low-metallicity stars than the higher mass galaxies, consistent with the findings of Kirby et al. (2008). He concludes that low-mass systems are expected to contribute substantively to the low-metallicity tail of the global MW halo MDF.

While our results seem to fit naturally into this picture, we note that the lowest mass MW satellites in the models of both groups have over an order of magnitude more stellar mass than the ultra-faint dwarfs, and models including lower luminosity systems are therefore still needed. However, the predicted trends between dwarf galaxy mass/luminosity, accretion time, $[\mathrm{Fe} / \mathrm{H}]$, and $[\alpha / \mathrm{Fe}]$ (Bullock \& Johnston 2005; Robertson et al. 2005; Johnston et al. 2008; Prantzos 2008) can still be used to infer that our observational results are broadly consistent with the presently favored cosmological model.

\section{CONCLUDING REMARKS}

We have presented a high-resolution chemical abundance analysis for six stars located in the ultra-faint dwarf galaxies Ursa Major II and Coma Berenices. Comparing these results with previous studies of stars in the MW halo and the brighter, classical dSphs, we arrive at the following conclusions. 
1. The ultra-faint dwarf galaxies contain significant numbers of extremely metal-poor stars with $[\mathrm{Fe} / \mathrm{H}]<-3$. Two out of the six stars in our sample (both in UMa II) fall into this category, despite our metallicity-independent selection criteria. Although the sample is obviously small, the statistics are inconsistent with the metallicity distributions of both the brighter dSphs and the MW halo. Schöerck et al. (2009) find that less than $2 \%$ of halo stars are at $[\mathrm{Fe} / \mathrm{H}]<-3$, so the probability of our sample containing two such stars is very low. In the brighter dSphs, not a single star out of 47 observed at high spectral resolution and several thousand observed at lower resolution has been found with $[\mathrm{Fe} / \mathrm{H}]<-3$. Thus, not only do the ultra-faint dwarf galaxies have lower mean metallicities than any other known stellar systems, but they also appear to contain a larger fraction of extremely metal-poor stars.

2. The ultra-faint dwarf galaxies have large internal metallicity spreads. Confirming the earlier results at lower spectral resolution of Simon \& Geha (2007), Kirby et al. (2008), and Norris et al. (2008), we find that our three stars in each galaxy span a range of $\sim 0.9$ dex in $[\mathrm{Fe} / \mathrm{H}]$ in UMa II and $\sim 0.6$ dex in ComBer. Again, despite the small samples, it is clear that there are significant internal metallicity variations in these objects. The metallicity spreads have important implications for the formation of the ultra-faint dwarf galaxies, suggesting either early star formation in multiple protodwarf galaxies that later merged, extended star formation histories, or incomplete mixing in the early ISM. Distinguishing between these scenarios requires larger samples of high-resolution spectroscopy of stars covering a wider range of metallicities and improved age constraints from photometric studies.

3. The abundance pattern of light elements $(Z<30)$ in the ultra-faint dwarf galaxies is remarkably similar to the $M W$ halo. In contrast to what is seen in the brighter dSphs (Venn et al. 2004, and references therein), we find that the trends of $\alpha$ and iron-peak abundances with $[\mathrm{Fe} / \mathrm{H}]$ in the ultra-faint dwarf galaxies are in excellent agreement with the best halo samples over the same metallicity range of $-3.2 \lesssim[\mathrm{Fe} / \mathrm{H}] \lesssim-2.34$. This result suggests that the metal-poor end of the MW halo population could have been built up from the destruction of large numbers of systems similar to the ultra-faint dwarf galaxies.

4. Neutron-capture elements, specifically $\mathrm{Sr}$ and Ba, have extremely low abundances in the ultra-faint $d w a r f$ galaxies. Particularly in ComBer, the $\mathrm{Sr}$ and $\mathrm{Ba}$ values observed are well below the abundances found in MW halo stars with similar Fe abundances. In UMa II, the neutron-capture abundances are lower than the halo averages, but not outside the distribution. Both galaxies exhibit a large scatter $(\sim 1$ dex) in the abundances of these elements. This abundance pattern may originate from the weak $r$-process, whose site is unknown but is speculated to be in very massive stars $\left(M \gtrsim 20 M_{\odot}\right)$.

5. The results above are broadly consistent with the predictions of the currently favored cosmological models (e.g., Bullock \& Johnston 2005; Robertson et al. 2005; Johnston et al. 2008; Prantzos 2008). While the majority of the mass in the stellar halo was formed in much larger systems, our results support a scenario where galaxies similar to the faintest dwarf galaxies may have been the source for much of the metal-poor end of the MW halo Fe metallicity distribution.
This study has provided the first evidence that the chemical evolution in two of the faintest dwarf galaxies known may have been similar to that of the MW halo. We find some intriguing and yet-to-be-explained abundance signatures, such as the low neutron-capture abundances, but overall there is a surprising level of agreement between the abundances in the ultrafaint dwarf galaxies and the most metal-poor halo stars (see Figure 10). Fully unraveling the complex relationship between the entire population of observed dwarf galaxies and the formation of the stellar halo of the MW, however, will require more spectroscopic and photometric data. It is not yet clear whether the differences we have found from previous studies of the brighter MW dSphs-the presence of extremely metalpoor stars in the ultra-faint dwarf galaxies and the agreement between the light-element abundance pattern in the ultra-faint dwarf galaxies and the halo-stem from actual differences between the classical dSphs and their much fainter cousins or observational biases. Spectroscopy of more metal-poor stars in the bright $\mathrm{dSphs}$ and more metal-rich stars in the ultra-faint dwarf galaxies (e.g., Koch et al. 2008b; I. I. Ivans et al. 2010, in preparation; J. Simon et al. 2010, in preparation) to increase the sample sizes and broaden the range of overlap between the two types of galaxies will help clarify this picture and provide further clues to the formation of the halo of our Galaxy.

Data presented herein were obtained at the W. M. Keck Observatory, which is operated as a scientific partnership among the California Institute of Technology, the University of California, and the National Aeronautics and Space Administration. The Observatory was made possible by the generous financial support of the W. M. Keck Foundation. The authors wish to recognize and acknowledge the very significant cultural role and reverence that the summit of Mauna Kea has always had within the indigenous Hawaiian community. We are most fortunate to have the opportunity to conduct observations from this mountain. We thank Evan Kirby for providing information on the stellar parameters, Kjell Eriksson for computing MARCS model atmospheres, Ian Roederer, Volker Bromm, and Grant Matthews for enlightening discussions about neutron-capture nucleosynthesis, and Chris Sneden, John Norris, and the anonymous referee for helpful suggestions regarding the manuscript. We are especially indebted to Xavier Prochaska for his extensive help with running the HIRES data reduction pipeline. A.F. acknowledges support through the Clay Fellowship administered by the Smithsonian Astrophysical Observatory and the W. J. McDonald Fellowship of the McDonald Observatory. J.D.S. gratefully acknowledges the support of a Millikan Fellowship provided by Caltech and a Vera Rubin Fellowship from the Carnegie Institution of Washington. B.W. thanks NSF AST 0908193 for support. M.G. thanks NSF 0908752 for support.

Facilities: Keck I (HIRES)

\section{REFERENCES}

Abazajian, K. N., et al. 2009, ApJS, 182, 543

Aoki, W., Beers, T. C., Christlieb, N., Norris, J. E., Ryan, S. G., \& Tsangarides, S. 2007a, ApJ, 655, 492

Aoki, W., Norris, J. E., Ryan, S. G., Beers, T. C., \& Ando, H. 2002, ApJ, 576, L141

Aoki, W., et al. 2005, ApJ, 632, 611

Aoki, W., et al. 2007b, ApJ, 660, 747

Aoki, W., et al. 2009, A\&A, 502, 569

Asplund, M., Grevesse, N., \& Sauval, A. J. 2005, in ASP Conf. Ser. 336, Cosmic Abundances as Records of Stellar Evolution and Nucleosynthesis, ed. T. G. Barnes III \& F. N. Bash (San Francisco, CA: ASP), 25

Barklem, P. S., et al. 2005, A\&A, 439, 129 
Baumueller, D., Butler, K., \& Gehren, T. 1998, A\&A, 338, 637

Beers, T. C., \& Christlieb, N. 2005, ARAA, 43, 531

Belokurov, V., et al. 2006, ApJ, 647, L111

Belokurov, V., et al. 2007, ApJ, 654, 897

Belokurov, V., et al. 2008, ApJ, 686, L83

Bonifacio, P., Hill, V., Molaro, P., Pasquini, L., Di Marcantonio, P., \& Santin, P. 2000, A\&A, 359, 663

Bonifacio, P., Sbordone, L., Marconi, G., Pasquini, L., \& Hill, V. 2004, A\&A, 414, 503

Bullock, J. S., \& Johnston, K. V. 2005, ApJ, 635, 931

Burris, D. L., Pilachowski, C. A., Armandroff, T. E., Sneden, C., Cowan, J. J., \& Roe, H. 2000, ApJ, 544, 302

Cayrel, R., et al. 2004, A\&A, 416, 1117

Chou, M.-Y., et al. 2007, ApJ, 670, 346

Christlieb, N., Gustafsson, B., Korn, A. J., Barklem, P. S., Beers, T. C., Bessell, M. S., Karlsson, T., \& Mizuno-Wiedner, M. 2004, ApJ, 603, 708

Clem, J. L. 2006, PhD thesis, University of Victoria, Canada

Cohen, J. G., \& Huang, W. 2009, ApJ, 701, 1053

Cohen, J. G., et al. 2005, ApJ, 633, L109

Collet, R., Asplund, M., \& Trampedach, R. 2006, ApJ, 644, L121

Font, A. S., Johnston, K. V., Bullock, J. S., \& Robertson, B. E. 2006, ApJ, 646, 886

François, P., et al. 2007, A\&A, 476, 935

Frebel, A., Christlieb, N., Norris, J. E., Aoki, W., \& Asplund, M. 2006a, ApJ, 638, L17

Frebel, A., Collet, R., Eriksson, K., Christlieb, N., \& Aoki, W. 2008, ApJ, 684 588

Frebel, A., Johnson, J. L., \& Bromm, V. 2007a, MNRAS, 380, L40

Frebel, A., Norris, J. E., Aoki, W., Honda, S., Bessell, M. S., Takada-Hidai, M., Beers, T. C., \& Christlieb, N. 2007b, ApJ, 658, 534

Frebel, A., et al. 2005, Nature, 434, 871

Frebel, A., et al. 2006b, ApJ, 652, 1585

Fulbright, J. P., Rich, R. M., \& Castro, S. 2004, ApJ, 612, 447

Geha, M., Willman, B., Simon, J., Strigari, L., Kirby, E., Law, L., \& Strader, J. 2009, ApJ, 692, 1461

Geisler, D., Smith, V. V., Wallerstein, G., Gonzalez, G., \& Charbonnel, C. 2005, AJ, 129, 1428

Geisler, D., Wallerstein, G., Smith, V. V., \& Casetti-Dinescu, D. I. 2007, PASP, 119,939

Gratton, R. G., Carretta, E., Eriksson, K., \& Gustafsson, B. 1999, A\&A, 350, 955

Gratton, R. G., Sneden, C., Carretta, E., \& Bragaglia, A. 2000, A\&A, 354, 169

Green, E. M., Demarque, P., \& King, C. R. 1984, BAAS, 16, 997

Gustafsson, B., et al. 2008, A\&A, 486, 951

Harbeck, D., Smith, G. H., \& Grebel, E. K. 2003, AJ, 125, 197

Harris, W. E. 1997, VizieR Online Data Catalog, 7202

Helmi, A., et al. 2006, ApJ, 651, L121

Hill, V., et al. 2002, A\&A, 387, 560

Honda, S., Aoki, W., Ishimaru, Y., \& Wanajo, S. 2007, ApJ, 666, 1189

Honda, S., Aoki, W., Ishimaru, Y., Wanajo, S., \& Ryan, S. G. 2006, ApJ, 643 , 1180

Irwin, M., et al. 2007, ApJ, 656, L13

Ivans, I. I., Simmerer, J., Sneden, C., Lawler, J. E., Cowan, J. J., Gallino, R., \& Bisterzo, S. 2006, ApJ, 645, 613

Ivans, I. I., Sneden, C., James, C. R., Preston, G. W., Fulbright, J. P., Höflich, P. A., Carney, B. W., \& Wheeler, J. C. 2003, ApJ, 592, 906

Izutani, N., Umeda, H., \& Tominaga, N. 2009, ApJ, 692, 1517

Johnston, K. V., Bullock, J. S., Sharma, S., Font, A., Robertson, B. E., \& Leitner, S. N. 2008, ApJ, 689, 936

Kim, Y.-C., Demarque, P., Yi, S. K., \& Alexander, D. R. 2002, ApJS, 143, 499

Kirby, E. N., Simon, J. D., Geha, M., Guhathakurta, P., \& Frebel, A. 2008, ApJ, $685, \mathrm{~L} 43$

Koch, A., Grebel, E. K., Gilmore, G. F., Wyse, R. F. G., Kleyna, J. T., Harbeck, D. R., Wilkinson, M. I., \& Wyn Evans, N. 2008a, AJ, 135, 1580

Koch, A., Grebel, E. K., Wyse, R. F. G., Kleyna, J. T., Wilkinson, M. I., Harbeck, D. R., Gilmore, G. F., \& Evans, N. W. 2006, AJ, 131, 895

Koch, A., McWilliam, A., Grebel, E. K., Zucker, D. B., \& Belokurov, V. 2008b, ApJ, 688, L13

Koposov, S., et al. 2008, ApJ, 686, 279

Kurucz, R. L. 1993, Kurucz CD-ROM 13, ATLAS9 Stellar Atmosphere Programs and $2 \mathrm{~km} \mathrm{~s}^{-1}$ Grid (Cambridge: SAO)

Kurucz, R. L. 1998, in IAU Symp. 189, Fundamental Stellar Properties, ed. T. R. Bedding, A. J. Booth, \& J. Davis (Dordrecht: Kluwer), 217

Lai, D. K., Bolte, M., Johnson, J. A., Lucatello, S., Heger, A., \& Woosley, S. E. 2008, ApJ, 681, 1524

Lucatello, S., Beers, T. C., Christlieb, N., Barklem, P. S., Rossi, S., Marsteller, B., Sivarani, T., \& Lee, Y. S. 2006, ApJ, 652, L37
Marsteller, M., Beers, T. C., Rossi, S., Christlieb, N., Bessell, M. S., \& Rhee, J. 2005, Nucl. Phys. A, 758, 312

Martin, N. F., de Jong, J. T. A., \& Rix, H.-W. 2008, ApJ, 684, 1075

Martin, N. F., Ibata, R. A., Chapman, S. C., Irwin, M., \& Lewis, G. F. 2007, MNRAS, 380, 281

Mateo, M. L. 1998, ARA\&A, 36, 435

McWilliam, A., Preston, G. W., Sneden, C., \& Searle, L. 1995, AJ, 109, 2757

McWilliam, A., Rich, R. M., \& Smecker-Hane, T. A. 2003, ApJ, 592, L21

Meléndez, J., \& Barbuy, B. 2009, A\&A, 497, 611

Meynet, G., Ekström, S., \& Maeder, A. 2006, A\&A, 447, 623

Monaco, L., Bellazzini, M., Bonifacio, P., Ferraro, F. R., Marconi, G., Pancino, E., Sbordone, L., \& Zaggia, S. 2005, A\&A, 441, 141

Muñoz, R. R., Carlin, J. L., Frinchaboy, P. M., Nidever, D. L., Majewski, S. R., \& Patterson, R. J. 2006, ApJ, 650, L51

Nissen, P. E., \& Schuster, W. J. 1997, A\&A, 326, 751

Nomoto, K., Hashimoto, M., Tsujimoto, T., Thielemann, F.-K., Kishimoto, N., Kubo, Y., \& Nakasato, N. 1997, Nucl. Phys. A, 616, 79

Nomoto, K., Tominaga, N., Umeda, H., Kobayashi, C., \& Maeda, K. 2006, Nucl. Phys. A, 777, 424

Norris, J. E., Christlieb, N., Korn, A. J., Eriksson, K., Bessell, M. S., Beers, T. C., Wisotzki, L., \& Reimers, D. 2007, ApJ, 670, 774

Norris, J. E., Gilmore, G., Wyse, R. F. G., Wilkinson, M. I., Belokurov, V., Wyn Evans, N., \& Zucker, D. B. 2008, ApJ, 689, L113

Norris, J. E., Ryan, S. G., \& Beers, T. C. 2001, ApJ, 561, 1034

Otsuki, K., Honda, S., Aoki, W., Kajino, T., \& Mathews, G. J. 2006, ApJ, 641, L117

Peñarrubia, J., Navarro, J. F., \& McConnachie, A. W. 2008, ApJ, 673, 226

Plez, B., Masseron, T., Van Eck, S., Jorissen, A., Coheur, P. F., Godefroid, M., \& Christlieb, N. 2008, Poster Presented in ASP Conf. 384, Cool Stars, Stellar Systems, and the Sun

Prantzos, N. 2008, A\&A, 489, 525

Qian, Y.-Z., \& Wasserburg, G. J. 2003, ApJ, 588, 1099

Robertson, B., Bullock, J. S., Font, A. S., Johnston, K. V., \& Hernquist, L. 2005, ApJ, 632, 872

Ryan, S. G., Norris, J. E., \& Beers, T. C. 1996, ApJ, 471, 254

Sadakane, K., Arimoto, N., Ikuta, C., Aoki, W., Jablonka, P., \& Tajitsu, A. 2004, PASJ, 56, 1041

Sakamoto, T., \& Hasegawa, T. 2006, ApJ, 653, L29

Schöerck, T., et al. 2009, A\&A, 507, 817

Searle, L., \& Zinn, R. 1978, ApJ, 225, 357

Shetrone, M., Venn, K. A., Tolstoy, E., Primas, F., Hill, V., \& Kaufer, A. 2003, AJ, 125,684

Shetrone, M. D., Bolte, M., \& Stetson, P. B. 1998, AJ, 115, 1888

Shetrone, M. D., Côté, P., \& Sargent, W. L. W. 2001, ApJ, 548, 592

Shetrone, M. D., Smith, G. H., Briley, M. M., Sandquist, E., \& Kraft, R. P. 1999, PASP, 111, 1115

Simmerer, J., Sneden, C., Cowan, J. J., Collier, J., Woolf, V. M., \& Lawler, J. E. 2004, ApJ, 617, 1091

Simon, J. D., \& Geha, M. 2007, ApJ, 670, 313

Smith, J. A., et al. 2002, AJ, 123, 2121

Sneden, C., Cowan, J. J., \& Gallino, R. 2008, ARA\&A, 46, 241

Sneden, C., Kraft, R. P., Guhathakurta, P., Peterson, R. C., \& Fulbright, J. P. 2004, AJ, 127, 2162

Sneden, C. A. 1973, PhD thesis, University of Texas at Austin

Spite, M., et al. 2006, A\&A, 455, 291

Strigari, L. E., Bullock, J. S., Kaplinghat, M., Simon, J. D., Geha, M., Willman, B., \& Walker, M. 2008, Nature, 454, 1096

Tollerud, E. J., Bullock, J. S., Strigari, L. E., \& Willman, B. 2008, ApJ, 688 277

Tolstoy, E., Hill, V., \& Tosi, M. 2009, ARA\&A, 47, 371

Tolstoy, E., Venn, K. A., Shetrone, M., Primas, F., Hill, V., Kaufer, A., \& Szeifert, T. 2003, AJ, 125, 707

Travaglio, C., Gallino, R., Arnone, E., Cowan, J., Jordan, F., \& Sneden, C. 2004, ApJ, 601, 864

Umeda, H., \& Nomoto, K. 2002, ApJ, 565, 385

Venn, K. A., Irwin, M., Shetrone, M. D., Tout, C. A., Hill, V., \& Tolstoy, E. 2004, AJ, 128, 1177

Vogt, S. S., et al. 1994, Proc. SPIE, 2198, 362

Walsh, S. M., Jerjen, H., \& Willman, B. 2007, ApJ, 662, L83

Wanajo, S., \& Ishimaru, Y. 2006, Nucl. Phys. A, 777, 676

Willman, B., et al. 2005a, AJ, 129, 2692

Willman, B., et al. 2005b, ApJ, 626, L85

Woosley, S. E., \& Weaver, T. A. 1995, ApJS, 101, 181

Zucker, D. B., et al. 2006a, ApJ, 650, L41

Zucker, D. B., et al. 2006b, ApJ, 643, L103 